\title{
NOVEL TECHNIQUES FOR SLURRY BUBBLE COLUMN \\ HYDRODYNAMICS
}

\author{
Final Technical Report
}

\section{Principal Investigators:}

\section{M.P. Dudukovic}

The Laura and William Jens

Professor and Chairman

Director, Chemical Reaction Engineering

Laboratory

\author{
Washington University \\ Department of Chemical Engineering \\ Campus Box 1198 \\ One Brookings Drive \\ St. Louis, Missouri 63130 \\ Fax: 314-935-4832 \\ Phone: 314-935-6021 \\ E-mail: dudu@wuche3.wustl.edu
}

Ohio State University

Department of Chemical Engineering

140 West $19^{\text {th }}$ Avenue-Room 125

Columbus, Ohio 43210-1180

Fax: 614-292-3769

Phone: 614-292-7907

E-mail: FAN@er6s1.eng.ohio-state.edu

Exxon Research and Engineering

P.O. Box 101

Florham Park, NJ 07931

Fax: 201-765-1189

Phone: 201-765-6109

E-mail: mchang@crsgil.erenj.com

\section{Co-Investigators}

Washington University: $\quad$ Dr. M. Al-Dahhan, Dr. J. Chen, P. Gupta, Dr. A. Kemoun, Dr. R. Mudde (Delft University), Dr. Y. Pan, S. Roy, Dr. Y. Yang

Ohio State University: Dr. P. Jiang, D.J. Lee, G. Shaver

Exxon Research and Engineering: $\quad$ Dr. C. Coulaloglou, Dr. W. Heard

\section{DRAFT}

May 14, 1999

Prepared for the United States Department of Energy

Award No. DOE-FG 22-95 PC 95212

Award Period: July 1, 1995 - June 30, 1998 


\section{Disclaimer}

This report was prepared as an account of work sponsored by an agency of the United States Government. Neither the United States Government nor any agency therefor, nor any of their employees, makes any warranty, express or implied, or assumes any legal liability or responsibility for the accuracy, completeness, or usefulness of any information, apparatus, product, or process disclosed, or represents that its use would not infringe privately owned rights. Reference herein to any specific commercial product, process, or service by trade name, trademark, manufacturer, or otherwise does not necessarily constitute or imply its endorsement, recommendation or favoring by the United States Government or any agency thereof. The views and opinions of authors expressed herein do not necessarily state or reflect those of the United States Government or any agency thereof. 


\title{
Novel Techniques for Slurry Bubble Column Hydrodynamics
}

\author{
Final Technical Report \\ DE FG 2295 PC 95212 \\ July 1, 1995 - June 30, 1998 \\ +9 month no cost extension
}

\begin{abstract}
The objective of this cooperative research effort between Washington University, Ohio State University and Exxon Research Engineering Company was to improve the knowledge base for scale-up and operation of slurry bubble column reactors for syngas conversion and other coal conversion processes by increased reliance on experimentally verified hydrodynamic models.

During the first year (July 1, 1995 - June 30, 1996) of this three year program novel experimental tools (computer aided radioactive particle tracking (CARPT), particle image velocimetry (PIV), heat probe, optical fiber probe and gamma ray tomography) were developed and tuned for measurement of pertinent hydrodynamic quantities, such as velocity field, holdup distribution, heat transfer and bubble size. The accomplishments were delineated in the First Technical Annual Report.

The second year (July, 1996 - June 30, 1997) was spent on further development and tuning of the novel experimental tools (e.g., development of Monte Carlo calibration for CARPT, optical probe development), building up the hydrodynamic data base using these tools and comparison of the two techniques (PIV and CARPT) for determination of liquid velocities. A phenomenological model for gas and liquid backmixing was also developed. All accomplishments were summarized in the Second Annual Technical Report.

During the third and final year of the program (July 1, 1997 - June 30, 1998) and during the nine months no cost extension, the high pressure facility was completed and a set of data was taken at high pressure conditions. Both PIV, CT and CARPT were used. More fundamental hydrodynamic modeling was also undertaken and model predictions were compared to data. The accomplishments for this period are summarized in this report.
\end{abstract}




\title{
NOVEL TECHNIQUES FOR SLURRY BUBBLE COLUMN HYDRODYNAMICS
} DE FG 22-95 PC 95212

\author{
Joint University/Industry Project \\ June 1, 1995 - July 30, 1998 \\ (+ 9 Months No-Cost Extension)
}

FINAL TECHNICAL REPORT

\section{TABLE OF CONTENTS}

Page No.

Disclaimer

Abstract

ii

Table Contents

List of Figures

List of Tables

Executive Summary

iii

iv

Vi

Xi

Xii

1.1 Bubble Columns (BC)/Slurry Bubble Columns (SBC) …..... 1

$1.2 \quad$ Focus of the Investigation ................................................. 3

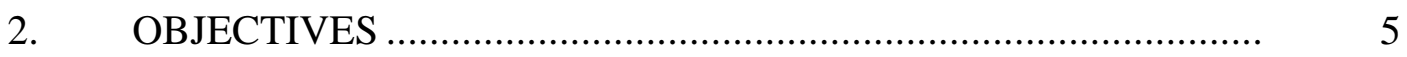

2.1 Review of the Overall Objectives and Accomplishments...... 5

2.2 Accomplishments During Year 1 ....................................... 7

2.3 Accomplishments During Year 2 ..................................... 7

2.4 Objectives and Accomplishments for Year 3....................... 8

3. GAS HOLDUP IN HIGH PRESSURE BUBBLE COLUMNS BY COMPUTED TOMOGRAPHY (CT)

3.1 Introduction and Objective ...................................................

3.2 The High Pressure Experimental Facility.............................. 10

3.3 Results and Discussion..................................................... 14

3.3.1 Radial Gas Holdup Distribution ................................. 14

3.3.2 Cross-Sectional Average Gas Holdup ........................ 17

3.4 Comparison with Various Correlations in the Literature ........ 21

3.5 Summary.................................................................. 26

3.6 Nomenclature ................................................................. 26

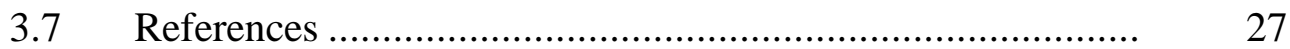


4. COMPUTER AUTOMATED RADIOACTIVE PARTICLE

TRACKING (CARPT) IN HIGH PRESSURE BUBBLE

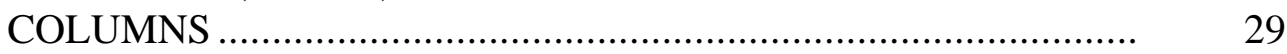

4.1 Introduction.................................................................. 29

4.2 High Pressure Experimental Set-up ...................................... 29

4.2.1. CARPT Facility .................................................... 29

4.2.2. Experimental Applications........................................ 30

$4.3 \quad$ Results and Discussion.................................................... 35

$4.4 \quad$ References .................................................................. 37

5. MONTE CARLO SIMULATIONS OF NaI (TI) SCINTILLATION DETECTORS FOR MULTIPHASE FLOW MAPPING AND VISUALIZATION USING CARPT ……………………….........

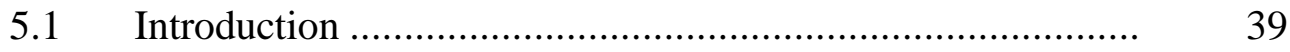

5.2 Research Objectives ........................................................ $\quad 40$

5.3. Mathematical Description ................................................. 41

5.3.1. Determination of Solid Angle .................................. 42

5.3.2. Photon Interaction with Reactor Media and Detector 45 Crystal

5.3.3. Computation of Simulated Counts ............................. 52

5.3.4. Optimization........................................................ 53

$5.4 \quad$ Theoretical Validation.......................................................... 54

5.5. Experimental Validation...................................................... 54

5.6. Conclusions .................................................................... 59

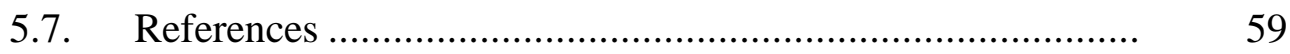

6. FLUID DYNAMICS IN HIGH PRESSURE SLURRY BUBBLE

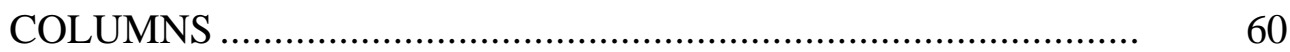

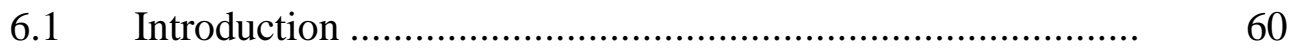

6.2 Experimental Set-up......................................................... 60

$6.3 \quad$ Single Bubble Rise Velocity.................................................. 61

6.4 Bubble Size Distribution ..................................................... 65

6.5 Theoretical Model and Numerical Method ........................... 69

6.5.1 Liquid Phase Model.................................................. 69

6.5.2 Gas Phase Model .................................................... 69

6.5.3 Dispersed Particle Model........................................... $\quad 70$

6.5.4 Coupling Among Individual Phases ........................... 71

6.6 Results from Numerical Study...........................................

6.6.1 Bubble Rising in Liquid-Solid Fluidized Medium..... $\quad 72$

6.6.2 Particle Entrainment ............................................... 72

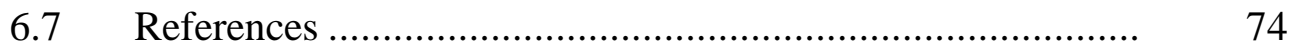


7. DYNAMIC SIMULATION OF BUBBLY FLOW IN BUBBLE COLUMN BY EULRIAN/EULERIAN METHOD ......................... $\quad 76$

7.1 Introduction .....................................................................

7.2 Ensemble Averaged Equations for Two-Phase Flow ............ $\quad 78$

7.3 Numerical Details ............................................................ 82

7.4 Large Structures in Bubble Columns...................................

7.5 Effects of Gas Distributors................................................ 92

7.6 Quantitative Comparison with Experiments ........................ 92

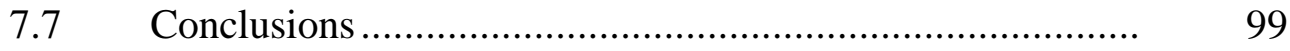

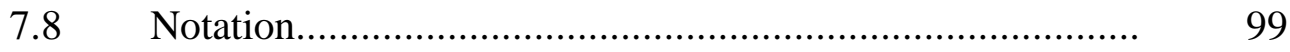

7.9 Literature Cited ............................................................. 100

8. SUMMARY AND CONCLUSIONS ……………………….......... 103 


\section{LIST OF FIGURES}

Figure No.

Caption

Page No.

Figure 1.1 Bubble Columns and Slurry Bubble Columns........................... 2

Figure 1.2 The Phenomena Occurring in Bubble and Slurry Bubble

Columns...

4

Figure 3.1 Flowsheet for High Pressure Column .................................... 11

Figure 3.2 Top View of the High Pressure Column; Side View of the High Pressure Bubble Column Elevation Along Section AA of Figure 3.2

Figure 3.3 Side View of the High Pressure Bubble Column Elevation Along Section AA of Figure 3.2

Figure 3.4 Perforated Plate Distributor of $0.04 \%$ Open Area

Figure 3.5 Radial Gas Holdup Distribution as a Function of Pressure for $\mathrm{U}_{\mathrm{g}}=2 \mathrm{~cm} / \mathrm{s}$

Figure 3.6 Radial Gas Holdup Distribution as a Function of Pressure for $\mathrm{U}_{\mathrm{g}}=12 \mathrm{~cm} / \mathrm{s}$.

Figure 3.7 Radial Distribution of Gas Holdup at Atmospheric Conditions as a Function of Superficial Gas Velocity for 19 cm Air-Water Column with Bubble Cap Distributor (Shollenberger et al., 1995)

Figure 3.8 Radial Distribution of Gas Holdup as a Function of Pressure for $\mathrm{Ug}=10 \mathrm{~cm} / \mathrm{s}$ (Taken from Shollenberger $e t$ al., 1996)

Figure 3.9 Cross-sectional Distribution of the Gas Holdup at (a) $\mathrm{P}=1$ atm, (b) $\mathrm{P}=3 \mathrm{~atm}$, and (c) $\mathrm{P}=7$ atm for $\mathrm{U}_{\mathrm{g}}=2 \mathrm{~cm} / \mathrm{s}$

Figure 3.10 Cross-sectional Distribution of the Gas Holdup at (a) $\mathrm{P}=1$ atm, (b) $\mathrm{P}=3 \mathrm{~atm}$, and (c) $\mathrm{P}=7$ atm for $\mathrm{U}_{\mathrm{g}}=12 \mathrm{~cm} / \mathrm{s}$

Figure 3.11 Cross-sectional Average Gas Holdup as a Function of Superficial Gas Velocity at Different Pressures

Figure 3.12 Cross-sectional Averaged Gas Holdup as a Function of Pressure at Different Superficial Gas Velocities 
Figure 3.13 Cross-sectional Average Gas Holdup as a Function of

Superficial Gas Velocity at Atmospheric Pressure.

Figure 3.14 Cross-Sectional Average Gas Holdup as a Function of

Superficial Gas Velocity at $\mathrm{P}=7$ atm

Figure 4.1 CARPT Setup for the High Pressure Bubble Column in

CREL

Figure 4.2 Calibration Device for High Pressure Bubble Column

Figure 4.3 Calibration Curves of Different Detectors

Figure 4.4 Tracer Particle Locations $(\mathrm{X}(\mathrm{t}), \mathrm{Z}(\mathrm{t}))$ During 20 Minutes of the Run.

Figure 4.5 Increased Accuracy Near the Sparger with Increased

Number of Detectors.

Figure 4.6 Axial Mean Velocities at Different z Level $(30$ and $90 \mathrm{~cm}$ )

for a Superficial Gas Velocity of $5 \mathrm{~cm} / \mathrm{s}$ at Pressure of 0.3

$\mathrm{MPa}$

Figure 4.7 Axial Liquid Velocity Profiles (a), Turbulent Kinetic

Energy (b) For an Atmospheric Column of 6 Inches in

Diameter

Figure 4.8 Kinetic Energy Profile. Superficial Gas Velocity: 5cm/s.

Pressure:0.3 Mpa.

Figure 5.1 Notation Used in the Selection of Angles for Monte Carlo

Calculations

Figure 5.2 Notation for the Case of a Point Source Located Directly

Above the Circular Face of the Detector.

Figure 5.3 The Four Possible Cases of Travel of Photons Through the

Detector

Figure 5.4 Schematic of the Experimental Setup to Verify the Monte

Carlo Simulations.

Figure 5.5 Spectrum Analysis of the Four Detectors used in the Experiments for Identification of the Threshold Signifying the Beginning of the Photopeak 
Figure 5.6 Reconstructed Particle Position Over 190 Data Points

Acquired Every $20 \mathrm{~ms}$

Figure 6.1 Effect of Pressure on Terminal Rise Velocity of Single

Bubbles in Paratherm NF Heat Transfer Fluid and

Predicted Values at (a) $27^{\circ} \mathrm{C}$ and (b) $78^{\circ} \mathrm{C}$

Figure 6.2 Comparisons of Measured and Calculated $R e$ of Single

Bubbles in Paratherm NF Heat Transfer Fluid Under

Varied Pressure and Temperature Conditions. The Fan-

Tsuchiya (1990) and Tomiyama et al. (1995) correlations

are Plotted (— and - - - , respectively) at Regular

Intervals of Mo Values. The Fan-Tsuchiya (1990)

Correlation at Measured Mo Values for Comparison With

Measured Re-Eo data (

Figure 6.3 Bubble Size Distributions Under Various Pressures at $T=$

$27^{\circ} \mathrm{C}$ and $U_{g}=8 \mathrm{~cm} / \mathrm{s}$

Figure 6.4 Bubble Size Distributions Under Various Pressures at $T=$

$27^{\circ} \mathrm{C}$ and $U_{g}=2 \mathrm{~cm} / \mathrm{s}$

Figure 6.5 Bubble Size Distributions Under Various Pressures at $T=$

$78^{\circ} \mathrm{C}$ and $U_{g}=5 \mathrm{~cm} / \mathrm{s}$

Figure 6.6 Simulation and Experimental Results of a Bubble Rising in a Liquid-Solid Fluidized Bed.

Figure 6.7 Simulation of a Bubble Emerging From a Liquid-Solid

Fluidized Bed

Figure 7.1 Computational Mesh Used For the Simulations of 2-D

Column With Discrete Gas Injectors

Figure 7.2 Instantaneous Contour Plots for Gas Holdup in (1) 11.2-cm wide Column at $\mathrm{U}_{\text {sup }}=1(\mathrm{Cm} / \mathrm{s})$; (2) $15.2-\mathrm{cm}$ wide Column at $\mathrm{U}_{\text {sup }}=1(\mathrm{Cm} / \mathrm{s})$; and (3) 32-cm wide Column at $\mathrm{U}_{\text {sup }}=1.2$ $(\mathrm{Cm} / \mathrm{s})$

Figure 7.3 The Instantaneous Flow Field in a 15-cm Wide Column at $\mathrm{U}_{\text {sup }}=1(\mathrm{~cm} / \mathrm{s})$. (1) Gas Holdup Contour; (2) Liquid

Velocity Vector; (3) Liquid Vorticity Contour.

Figure 7.4 Instantaneous Gas Holdup Contours in a $15.2 \mathrm{~cm}$ Wide Column Operated at (1) $U_{\text {sup }}=0.25 \mathrm{~cm} / \mathrm{s}$; (2) $U_{\text {sup }}=1 \mathrm{~cm} / \mathrm{s}$;

(3) $U_{\text {sup }}=2 \mathrm{~cm} / \mathrm{s}$. 
Figure 7.5 Spatial Power Spectra of $\mathrm{u}$ in a 15-cm Wide Column at Three Different Superficial Gas Velocities

Figure 7.6 Time Series of the Liquid Velocity at the Central Point of $15-\mathrm{cm}$ Wide Column at $\mathrm{U}_{\text {sup }}=1 \mathrm{~cm} / \mathrm{s}$

Figure 7.7 Temporal Power Dpectra of the Liquid Velocity in a 15-cm Wide Column at $\mathrm{U}_{\text {sup }}=1 \mathrm{~cm} / \mathrm{s}$

Figure 7.8 The Instantaneous Gas Holdup Contour of 15-cm Wide Column at $\mathrm{U}_{\text {sup }}=1 \mathrm{~cm} / \mathrm{s}$ with: (1) 1-jet; (2) 3-jet; (3) 9-jet;

(4) Uniform Gas Distributors.

Figure 7.9 Time Averaged Profiles of Gas Holdup and the Vertical Liquid Phase Velocity at the Middle Section of a $15-\mathrm{cm}$ wide Column at $\mathrm{U}_{\text {sup }}=1(\mathrm{~cm} / \mathrm{s})$, With Different Gas Distributors

Figure 7.10 Time-Averaged Velocity Profiles for the Middle Section of $11-\mathrm{cm}$ Wide Column at $\mathrm{U}_{\text {sup }}=1(\mathrm{~cm} / \mathrm{s})$

Figure 7.11 Time-Averaged Velocity Profiles for 15-cm Wide Column at $\mathrm{U}_{\text {sup }}=1 \mathrm{~cm} / \mathrm{s}$. Top: Upper Section; Middle: Middle Section; Bottom: Lower Section. — and $\bullet$ are the Numerical Prediction and Experimental Data, Respectively

Figure 7.12 Time-Averaged Velocity Profile for the Middle Section of a 32-cm Wide Column at $\mathrm{U}_{\text {sup }}=1.9 \mathrm{~cm} / \mathrm{s}$

Figure 7.13 Turbulence Intensities and Reynolds Shear Stress in the Middle Section of $15-\mathrm{cm}$ wide Column Operated at $\mathrm{U}_{\text {sup }}=1$ $\mathrm{cm} / \mathrm{s}$. - is the Numerical Values; $\bullet$ is the Experimental Data by Mudde et al. (1997) 


\section{LIST OF TABLES}

Table No.

Caption

Page No.

Table 3.1 Operating Conditions for the High Pressure System

Table 3.2 Cross-Sectional Average Gas Holdup at Different

Operating Conditions

Table $3.3 \quad$ Correlations for Gas Holdup.......................................... 22

Table 3.3 Correlations for Gas Holdup (Continued) ............................... 23

Table 3.4 Comparison of Cross-Sectional Average Gas Holdup with

Predictions of Different Correlations (and Percent Error in

Predictions)

Table 4.1 Operating Conditions for the High Pressure System .................. 30

Table 5.1 Comparison of Beam's et al. Formulation to the

Formulation Used in This Work

52

Table $7.1 \quad$ Column Size and Flow Conditions …...................................... 99 


\title{
NOVEL TECHNIQUES FOR SLURRY BUBBLE COLUMN HYDRODYNAMICS
}

\author{
FINAL TECHNICAL REPORT \\ June 1, 1995 - July 30, 1998 \\ (+ 9 Months No-Cost Extension)
}

\section{EXECUTIVE SUMMARY}

The objective of this cooperative University-Industry research effort between Washington University (WU), Ohio State University (OSU) and Exxon Research and Engineering Company (ER\&E) was to improve the basis for scale-up and operation of slurry bubble column reactors for syngas conversion and other coal conversion processes by increased reliance on experimentally verified hydrodynamic models.

The emphasis during this first year of this three year project was placed on developing the experimental techniques that are capable of providing accurate measurements of hydrodynamic quantities such as velocities and holdup distribution at operating conditions of interest. The accomplishments of Year 1 were described in the First Technical Annual Report and can be summarized as follows.

At Washington University (WU) the unique Computer Aided Radioactive Particle Tracking (CARPT) technique was modified and improved. The technique is capable of providing solids (or liquid) velocities at high gas or solids holdup where all other methods fail. Wavelet filtering of particle position versus time data was implemented, and this improved by an order of magnitude the accuracy of the turbulence parameters that the technique can provide. A Monte Carlo based modeling for calculation of detector responses for any position of the tracer was initiated. Data obtained with CARPT for liquid velocities was compared to the velocity estimates generated by heat pulse probe (HP), and it was shown that the later technique (HP) provides reliable local velocity information only in bubbly flows while it underestimates the velocities substantially during turbulent flow, i.e at high gas superficial velocity.

At Ohio State University (OSU) the limitations of the very powerful Particle Image Velocimetry (PIV) technique were explored and it was found that the technique has the potential of providing the full velocity and holdup field in columns of up to 4" in diameter. Experiments were initiated to compare PIV results in such a 4" column to the CARPT data. A new and useful probe for evaluation of local instantaneous heat transfer coefficient was developed at OSU and its capabilities demonstrated at ER\&E.

At Exxon Research and Engineering (ER\&E) preparations were made for making the high pressure 6" diameter bubble column available for CARPT and other measurements. Necessary modifications to the column were designed. Jointly with WU needed tracer particle strength was determined and a device for calibration was designed. In addition, CFDLIB codes for hydrodynamic modeling have been installed and a preliminary case 
study of a two dimensional column was conducted. It was shown that experimental time averaged velocity profiles qualitatively agree with the model predictions.

The emphasis during this second year of the project was: i) on further refinement and development of experimental techniques needed in obtaining reliable flow dynamic parameters in bubble columns, ii) on verification of these techniques, iii) on development of phenomenological models for bubble columns, iv) on testing of fundamental computational fluid dynamics models in predicting liquid flow patterns in bubble columns and v) on preparing a facility for high pressure studies in a six inch diameter column. The accomplishments of Year 2 were described in the Second Annual Technical Report and can be summarized as follows.

The CARPT technique was fully validated by a joint effort between WU and OSU. The comparison of PIV and CARPT data, taken on the same 4" diameter column, revealed that the two techniques generate essentially the same liquid time averaged velocities and the same order of magnitude of turbulence parameters at low superficial gas velocity when gas holdup is low. At such conditions both techniques re reliable. At high gas velocities that lead to churn turbulent flow, the results produced by the two techniques start to diverge somewhat, but the discrepancies can be fully accounted for on physical grounds (e.g. too short an interval of collection of PIV data for proper averaging, errors in PIV due to enhanced gas holdup, inability of CARPT to capture small scales of turbulence). It was concluded that the two techniques complement each other well. The data obtained from CARPT was also compared to Laser Doppler Anemometry (LDA), and Hot Film Anemometry (HFA) data and reasonable agreement was found. This validated the use of CARPT in churn turbulent flows, which are of industrial interest, and in which other techniques cannot provide the desired information.

A light transmittance optical probe was developed at OSU and its usefulness in obtaining information on bubble passing frequencies in opaque flow was successfully demonstrated. An optical reflectance probe was developed by Delft University for the measurement of bubble sizes and was successfully tested at WU in bubbly flows.

Based on the developed information on gas holdup profiles by Computed Tomography (CT) and based on liquid velocity patterns observed by CARPT, an engineering type model was developed at WU to represent flow, and mixing of each phase (liquid and gas) and transport between phases in bubble columns. The features and governing equations of the model were reported as well as the relationship of model parameters to the existing knowledge base.

The Computational Fluid Dynamics (CFD) work at WU and ER\&E resulted in successful simulation of the most often quoted and reported experiments in two-dimensional bubble columns which reveal a meandering structure and two streaks of downward moving vortices on each side of the meandering high holdup structure. It was also shown (WU and ER\&E) that the meandering plume observed experimentally in a partially aerated column can be captured by CFD while using relatively simple closure forms. 
The planned high pressure experiments in large diameter bubble columns (6") were rescheduled to be conducted at Washington University. This change was necessitated by the fact that it proved impossible for WU to conduct the originally planned radioactive particle experiments at Florham Park due to safety and licensing issues that were not foreseen. To remedy for this, ER\&E provided the additional funds needed to design and operate a high pressure facility at WU. The design of this facility was completed during the second year of the project. At that point it also became clear that a nine month no cost extension was needed to install and test the new facility and conduct the planned work on it.

During the final year 3 of the contract, and during the 9 month no-cost extension period, the necessity of which was explained above, the focus of the research was to implement the high pressure facility, collect and interpret data at high pressure and perform a quantitative comparison between CFD model predictions and data. The accomplishments achieved during this final period of the contract are described in this report and are summarized below.

A high pressure 6" diameter column was installed and operated at Washington University. Computer Tomography (CT) measurements revealed that the effect of pressure on gas holdup profiles is negligible in bubbly flows at low superficial gas velocities. At high superficial gas velocities, at which the flow at atmospheric pressure is churn turbulent, increased pressure causes a flattening in gas holdup profiles and a rise in overall holdup. This is apparently caused by the ability to maintain bubbly flow at higher superficial gas velocities at elevated pressures and delay transition to churn turbulent flow. Available correlations that can predict the observed mean holdup at atmospheric and elevated pressures were identified and reported. The details of the holdup measurements are described in Chapter 3 of this report.

The Computer Automated Radioactive Particle Tracking (CARPT) runs at WU in a 6" diameter column at elevated pressure reveal the same single cell liquid recirculation pattern in the time averaged sense, with the liquid rising in the middle of the column and falling down by the walls. The distributor zone where entry effects are notable is, however, much expanded at higher pressure. The magnitude of liquid recirculation velocities in the fully flow developed region is considerably reduced at elevated pressure compared to the results obtained at the same gas superficial velocity at atmospheric pressure. Details are provided in Chapter 4 of this report.

The sophisticated Monte Carlo procedure for calibration of the detectors for the CARPT experiments and for identification of the particle position during the CARPT experiments has been completed at WU and successfully tested against experimental data. Details are provided in Chapter 5 of this report. The availability of the Monte Carlo technique assures more extensive use of CARPT in the future.

Fluid dynamic behavior of small diameter (up to 4") high pressure columns was studied extensively by PIV and other techniques at OSU. Valuable correlations were developed for the bubble rise velocity. The data base for bubble size distributions was augmented 
and it clearly establishes the narrowing of the bubble size distribution and the shift to smaller bubble sizes at elevated pressure. Sophisticated modeling, based on first principles, of the shape and rise velocity of a bubble in a solid-liquid suspension was successfully completed and good comparison obtained between experimental results and model predictions. A comprehensive description is provided in Chapter 6 of this report.

Our combined effort (WU and ER\&E) to describe the flow field in the whole bubble column by computational fluid dynamics (CFD) focused on the use of Euler-Euler k-fluid model in the framework of the CFDLIB code of Los Alamos. Only the Euler-Euler based code was deemed practical for description of the flow field in the whole column. In this project we wanted to demonstrate that Euler-Euler approach is capable of predicting experimental observations even at low gas holdup in bubbly flow, which is the most severe test of the model since by definition the Euler-Euler approach is ill-suited for predicting the flow field caused by a single bubble or at vanishingly small gas holdup. At such low holdup conditions the predictions of CFDLIB (WU and ER\&E) were compared to the best PIV data available (OSU) in two dimensional beds. Very good comparison was obtained between predicted and measured time averaged liquid velocities. All the observed dynamic features of all the large structures of the flow were predicted well. The computed turbulence parameters were of the same order of magnitude and exhibited the same trends as data and the existing discrepancies can be fully rationalized based on physical arguments. This successful verification of CFD Euler-Euler approach for bubbly flow provides an increased motivation for its implementation for 3D simulations and for churn-turbulent flows. The detailed comparison between CFD predictions and PIV data in 2D columns is presented in Chapter 7 of this report. 


\section{INTRODUCTION AND MOTIVATION}

Synthesis gas from coal is one of the most abundant and reliable sources of energy and chemicals. From the heat transfer and high volumetric productivity viewpoints, bubble columns (BC) and slurry bubble columns (SBC) operated at high gas velocities in churn turbulent flow regime are the reactors of choice for a variety of synthesis gas conversion processes.

There is hardly any data in the flow regime, neither are there any fundamentally based design and scale-up procedures. In response to this need the Department of Energy (DOE) initiated the Fluid Dynamics Initiative which focuses on advancing the state of the art in understanding the fluid dynamics of bubble columns/slurry bubble columns and replacing empirical design methods with a more rational approach.

As part of this initiative DOE awarded the DE-FG22-95 PC 95212 grant for a collaborative effort between the Chemical Reaction Engineering Laboratory (CREL Washington University (WU), Ohio State University (OSU) and Exxon Research and Engineering (ER\&E) to advance the understanding of the hydrodynamics of $\mathrm{BC} / \mathrm{SBC}$ with the overall objectives to:

i) develop and verify accurate experimental techniques for obtaining important fluid dynamics parameters,

ii) generate database and expand it to high pressure systems,

iii) develop a set of phenomenological models and correlations to utilize this database,

iv) utilize the data to verify the fundamental computational fluid dynamics (CFD) models by testing different closure forms.

This grant enabled a unique integration of the expertise of the two universities (WU and OSU) and industry (ER\&E) toward achieving the goals set by the DOE Hydrodynamics Initiative. This study complemented well the work in progress at CREL-WU and OSU, Conract NO. DE-FC22-95 PC 95051, related to the LaPorte Advanced Fuels Demonstration Unit (AFDU) operated by Air Products with Department of Energy funding.

\subsection{Bubble Columns (BC/Slurry Bubble Columns (SBC)}

A bubble column (BC), shown in Figure 1.1, is a cylindrical vessel in which gas is sparged through a batch liquid or a cocurrently or countercurrently flowing liquid. The gas superficial velocities are order of magnitude larger than those of the liquid so that it is the gas that governs the fluid dynamics of the system. Small slurry particles in case of slurry bubble column (SBC), follow the liquid. It is well known that fluid dynamics (phase velocities and holdup distribution) affect the phase mixing and transport between the phases (gas-liquid interfacial area, transport coefficients) and, hence, to a large extent affect the conversion and selectivity. If the reaction involves phase volume changes (e.g., gas is either consumed or produced), the phase mixing, and transport parameters are affected along the column as illustrated in the flow chart shown in Figure 1.1. Thus, quantitative knowledge of fluid dynamics is essential for rational and predictive reactor design and scale-up. 

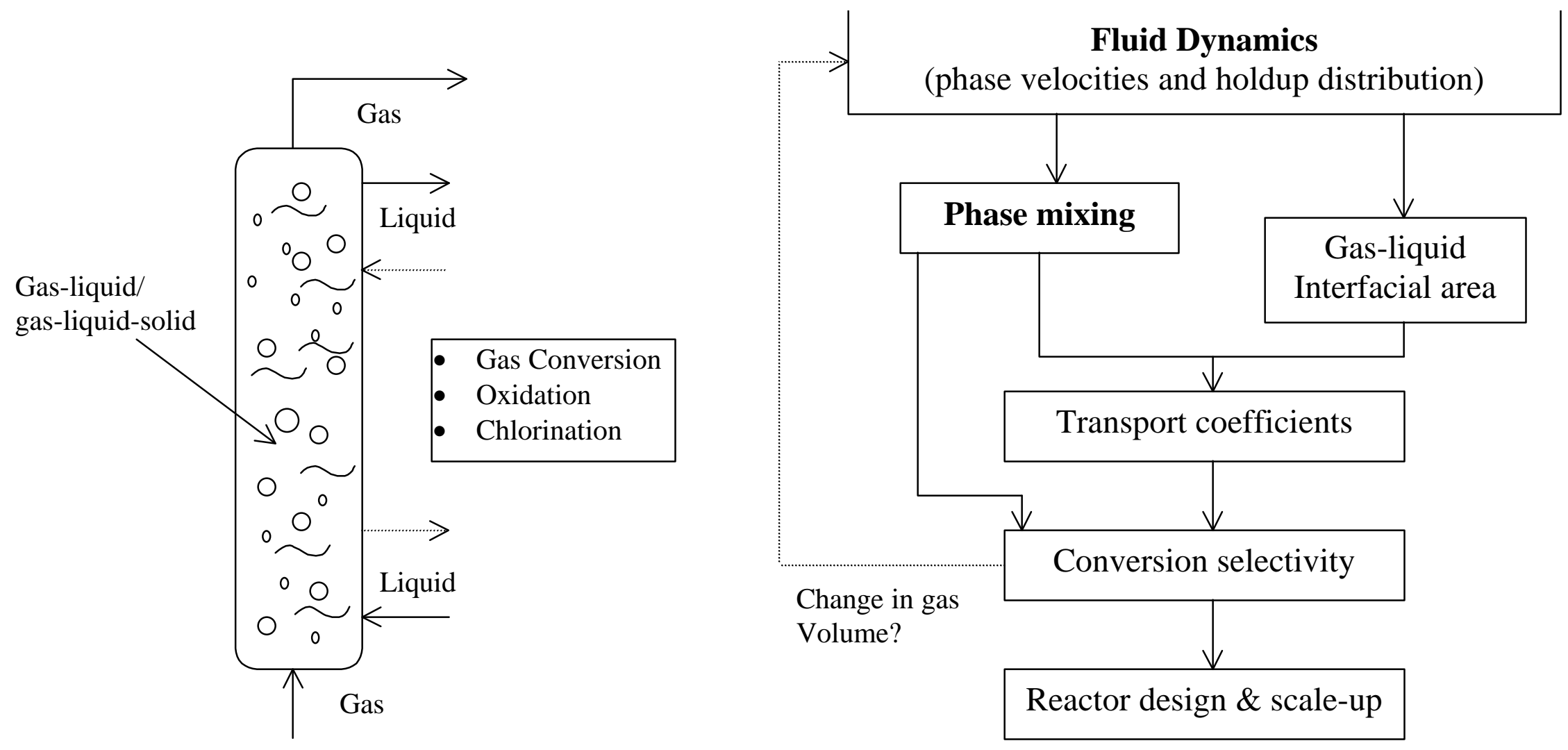

Figure 1.1: Bubble Columns and Slurry Bubble Columns 


\subsection{Focus of the Investigation}

The many phenomena occurring in $\mathrm{BC} / \mathrm{SBC}$, as illustrated in Figure 1.2, are very complex due to the intrinsic dynamic behavior of the dispersed phase motion and associated interactions. Physical and thermodynamic properties, operating variables and design parameters affect the fluid dynamics and transport phenomena in the column which together with kinetics determine the reactor performance (conversion and selectivity).

Our goal is to provide the macro scale description of the fluid dynamics phenomena in $\mathrm{BC} / \mathrm{SBC}$ and to attempt to relate the micro scale and the effects of various operating and design variables on the macro scale circulation, mixing and transport.

The investigated parameters and the techniques used to study the macro scale hydrodynamics under this grant are illustrated in Figure 1.2. This figure demonstrates also the integration of the expertise of Washington University, Ohio State University and Exxon Research \& Engineering in studying the fluid dynamics of bubble columns.

According to the main objectives of this grant, the following phenomena and variables need to be investigated (Figure 1.2) and quantified:

- Holdup distribution via Computer Tomography, CT - (WU) and Particle Image Velocimetry (PIV) - (OSU).

- Liquid circulation via Computer Automated Radioactive Active Particle Tracking, CARPT - (WU) and PIV - (OSU).

- Liquid turbulence and backmixing via CARPT - (WU) and PIV - (OSU).

- Bubble size distribution and bubble rise velocity via optical probes - both reflectance (WU) and transmittance (OSU) probes.

- Effect of gas flow rate and reactor pressure on the fluid dynamics (WU, ER\&E, OSU).

Along the process of developing and verifying the advanced experimental techniques used in this research, the database for the fluid dynamic parameters is expanded and utilized to build phenomenological models, correlations and also test fundamental fluid dynamics models. Along these lines we have focused on the following:

- Development of a phenomenological model for gas phase mixing (WU).

- Testing different closure forms by comparing the predictions of computational fluid dynamics models (CFD) with the data obtained by the above techniques (WU, ER\&E). 


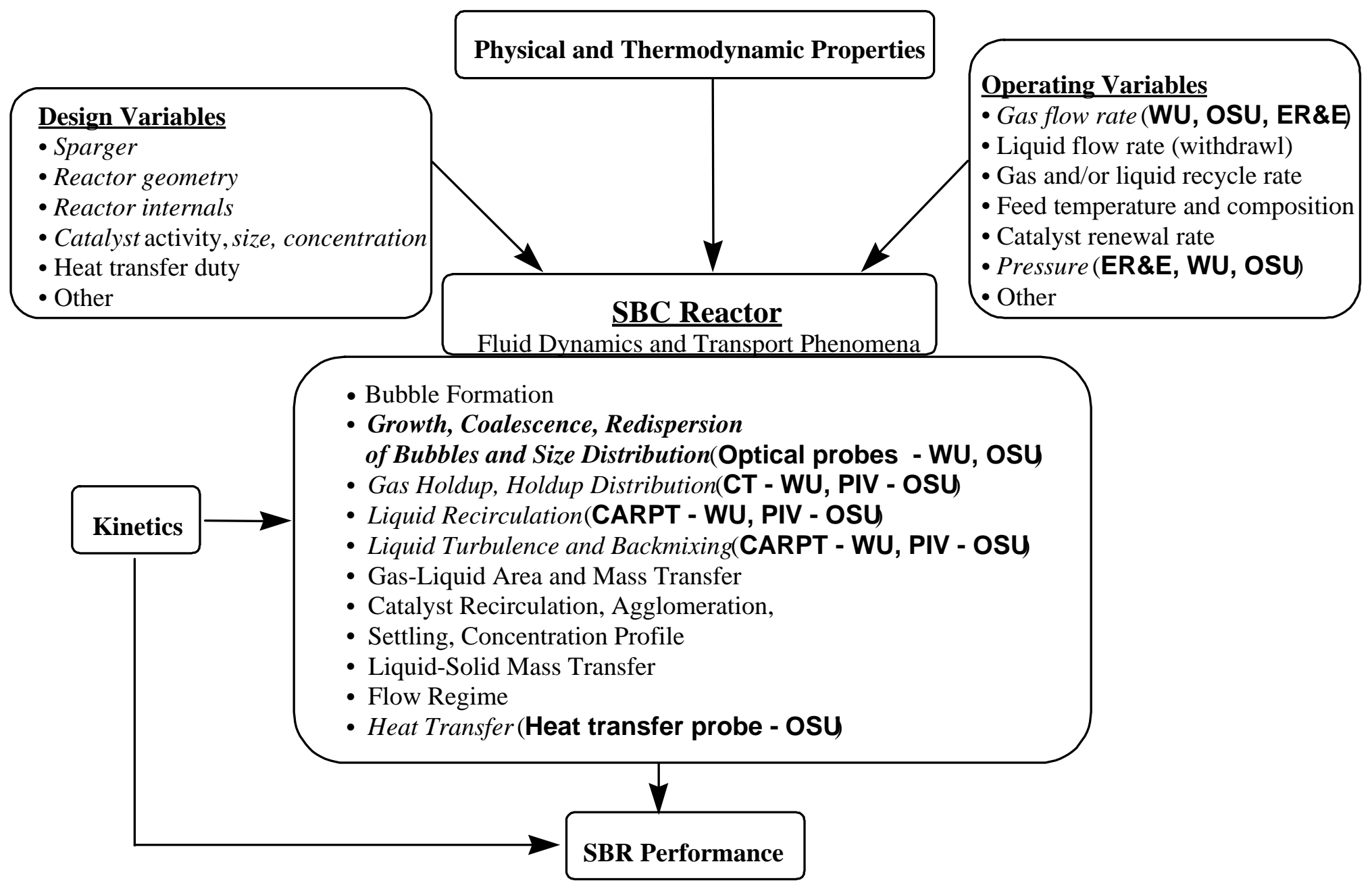

* Closure schemes for CFD simulations validated against experimental data from above techniques.

Figure 1.2: The Phenomena Occurring in Bubble and Slurry Bubble Columns 


\section{OBJECTIVES}

\subsection{Review of the Overall Objectives and Accomplishments}

The overall objectives of this cooperative University (Washington University and Ohio State University) - Industry (Exxon Research and Engineering) research was to improve the basis for scale-up and operation of bubble column/slurry bubble column reactors (BC/SBC) by an increased reliance on phenomenologically or fundamentally based hydrodynamic models which are experimentally verified. The research under this grant focused at providing experimental tools for measurement of important fluid dynamic quantities.

Specifically the following were set:

Task 1. Develop computerized mathematical procedure (based on physics of radiation and Monte Carlo calculations) for calibration of the Computer Automated Radioactive Particle Tracking Technique (CARPT) that will eliminate the currently lengthy in-situ and allow the technique to be used in the field on high pressure units. Specifically, plan the use of CARPT on a high pressure bubble column.

Task 2. Improve the accuracy of the CARPT technique and compare the velocities obtained by CARPT in certain regions of the column to those determined by the Heat Transfer Probe and Particle Image Velocimetry (PIV).

Task 3. Develop local probes for accessing velocities, holdups and heat coefficients in bubble column reactors.

Task 4. Collect velocity and voidage profile data in gas-liquid and gas-liquid-solid systems at different solids loadings and at close to atmospheric pressure.

Task 5. Use state-of-the-art hydrodynamic models and codes to predict velocity and holdup fields under conditions studied experimentally. Search for more suitable constitutive forms (e.g., lift, drag, turbulence closure models, etc.) to reach agreement between calculated and experimentally observed values. Try to assess the effect of elevated operating pressure on various constitutive forms.

Task 6. Collect the data in a high pressure 6" diameter bubble column and compare to model predictions. Also collect high pressure PIV data in slurry systems at Ohio State University. Refine models if needed.

All of the above tasks have been successfully completed as documented in our technical reports. The development of the Monte Carlo scheme and algorithm (Task 1) for calibration of the CARPT technique proved to be a rather involved and sophisticated endeavor. The progress made at Washington University (WU) in developing this powerful approach was summarized in the Second Annual Technical Report and the final 
implementation is summarized in this report. CARPT has also been employed successfully in a 6" high pressure bubble column as planned. The interactions with Exxon Research and Engineering (ERE) were invaluable in developing the high pressure system.

The accuracy of the CARPT technique was improved early on by implementing a wavelet based filter for minimization of the spurious velocities (First Annual Technical Report) and an additional gain in accuracy is achieved now with the final implementation of the Monte Carlo calibration procedure (this report). This work was completed at Washington University (WU). The liquid velocity data obtained by CARPT at WU at a specified set of operating conditions in bubbles columns of different diameter were compared with the data collected by PIV on the same columns at identical conditions at Ohio State University (OSU). Very good agreement between ensemble (time) averaged velocities obtained by the two techniques was found and reported (Second Annual Technical Report). It was also shown that the two techniques well complement each other with PIV providing more detailed information about the microstructure of the flow and CARPT providing information on the large scale flow pattern and large scale turbulence. The limited range over which the heat transfer probe can be used to locally determine the liquid velocity was also reported. This work at WU and OSU completed the objectives set by Task 2 .

The development and testing of local probes for assessment of velocities, holdups and heat transfer coefficients was pursued through out the duration of this grant at OSU, WU and ER\&E. The results obtained with the heat transfer probe and various optical probes were reported in the previous two annual technical reports fulfilling Task 3.

A database for liquid velocity profiles and voidage profiles was expanded (Task 4) both at OSU at column diameters lass than 4" and at WU in columns between 4" and 18" in diameter.

Our ultimate goal (Task 5) was to test the available models for computational fluid dynamics based on the ensemble averaged Eulerian k-fluid model to predict the experimental data for liquid velocities and gas holdup profiles. This task has been arduous as it requires considerable computational time and testing of various closure forms for interphase interaction terms. While pursuing this work we have also outlined as an interim solution a phenomenological model for assessing gas and liquid phase mixing (Second Annual Report). This model relies on the collected liquid velocity - gas holdup profiles data base and uses extrapolations to operating conditions. We at WU have also established a successful comparison of the predictions of the Euler k-fluid model, run in the CFDLIB framework of Los Alamos, and PIV data collected at OSU. This comparison is summarized in this report. The validity of CFD models for churnturbulent and gas-liquid-solid flows remains to be established.

High pressure data have been collected at OSU in a slurry system and the findings are presented in this report. At WU the high pressure facility for a 6" diameter column has 
been completed and holdup and CARPT data at elevated pressure was obtained and is presented in this report. This accomplishes Task 6.

\subsection{Accomplishments During Year 1}

The first year was dedicated to the development and improvement of experimental techniques. Major accomplishments were as follows:

- The modification of Computer Automated Radioactive Particle Tracking (CARPT) and Computed Tomography (CT) facilities for improved accuracy (WU). This included the implementation of wavelet based filtering and energy thresholding for significant reduction in spurious velocities.

- The work on Monte Carlo based simulation of CARPT calibration and the mathematical approach worked out (WU).

- The development of a special heat transfer probe to measure instantaneous local heat transfer coefficients in the vicinity of a single large passing bubble (OSU). This included:

- Validation of the reliability and accuracy.

- Evaluation of probe's capabilities for use in high pressure systems.

- The Computational Fluid Dynamic (CFD) simulation of a 2D bubble column was performed based on the CFDLIB algorithm of Los Alamos and utilizing the ensemble averaged Eulieran k-fluid model. (WU and ERE). This included:

- The evaluation of various closure schemes in CFD modeling such as mixing length and kinematic turbulent viscosity.

- The preparation for experiments in a high pressure 6" slurry bubble column. (ER\&E and WU) was initiated. This included:

- The computation of particle source strength for CARPT experiments.

- The design of a calibration device for the high pressure 6" slurry bubble column.

\subsection{Accomplishments During Year 2}

During Year 2, the focus was on further improvement of experimental techniques and their testing; comparison of velocity, holdups and other fluid dynamic quantities obtained by different techniques; further model development and CFD; and preparations for high pressure studies. The accomplishments were as follows:

- The development of the extensive programs required for Monte Carlo calibration of CARPT was completed (WU). 
- The comparative hydrodynamic study of a 4" bubble column using CARPT and Particle Image Velocimetry (PIV) was completed (WU, OSU).

- CARPT measurements were compared with other techniques such as Heat Pulse Anemometry (HPA), Laser Doppler Anemometry (LDA), Hot Film Anemometry (HFA) (WU).

- Progress was made in the development of transmittance and reflectance optical probes for gas holdup and bubble size measurements in churn turbulent flows (OSU, WU, ER\&E).

- Various closure schemes in the CFD simulations of bubble column fluid dynamics were tested (WU, ER\&E).

- The design of a high pressure 6" slurry bubble column at Washington University was completed (WU, ER\&E).

\subsection{Objectives and Accomplishments for Year 3}

The objectives set for Year 3 were as follows:

- Develop high pressure/high capacity 6" slurry bubble column facility at Washington University (WU, ER\&E).

- Monte Carlo simulations for CARPT calibrations and experimental verification (WU, ER\&E).

- Expand the experimental database to high pressure systems and gas-liquid-solids systems using PIV (OSU) and CARPT/CT (WU).

- Test the predictions from CFD models against CARPT, CT and PIV data using various closure schemes (WU, ER\&E).

To facilitate the review of the accomplishments achieved during year 3 (and during the follow-up no-cost extension period) of the grant the rest of this report is organized in self contained chapters.

Chapters 3 and 4 describe the work done to extend the CT/CARPT techniques to high pressure 6" diameter bubble columns (WU, ER\&E), respectively.

Chapter 5 outlines in detail the implementation of the Monte Carlo procedure for CARPT (WU).

Chapter 6 presents the slurry data at high pressure in smaller diameter columns (OSU, ER\&E).

Chapter 7 outlines the comparison of data and CFD predictions for bubbly flows (WU, ER\&E). 


\section{GAS HOLDUP IN HIGH PRESSURE BUBBLE COLUMNS BY COMPUTED TOMOGRAPHY}

\subsection{Introduction}

Two-phase bubble columns as well as three phase slurry bubble columns of various configurations have gained considerable attention in chemical industry due to their use in a number of processes, such as, Fischer-Tropsch synthesis, liquid phase methanol synthesis, wet oxidation of heavily polluted effluents, hydrogenation of heavy oils, etc. All of these processes operate at elevated pressure conditions (Deckwer, 1992). Most research on bubble columns has been performed at atmospheric conditions, and the scale-up and design of bubble columns at elevated pressures most often utilize the correlations that are based on data collected extensively at atmospheric conditions. This raises a question as to whether one can rely solely on the database established at atmospheric pressure. Jiang et al. (1995) observed that as pressure increases, the bubble size decreases, and the bubble size distribution becomes narrower. In an earlier study, Idogawa et al. (1986) observed that the behavior of bubbles depends closely on the type of gas distributor, and that this dependence weakened as the pressure increased. The effect vanished above $10 \mathrm{MPa}$. In the second study, Idogawa et al. (1987) reported that pressure had no effect on bubble diameter in bubble columns with gas superficial velocity in the range of 0.5 to $5.0 \mathrm{~cm} / \mathrm{s}$. In addition, Kölbel et al. (1961) found that gas holdup in a bubble column with a porous plate distributor was not affected by pressure in the range $0.1 \mathrm{MPa}$ to $1.6 \mathrm{MPa}$ when the superficial gas velocity, evaluated at the pressure in the column, was less than $3 \mathrm{~cm} / \mathrm{s}$. Deckwer et al. (1980) measured gas holdup in a slurry bubble column with a porous plate distributor containing fine particles at pressures up to $1.1 \mathrm{MPa}$ and with superficial gas velocity below $4 \mathrm{~cm} / \mathrm{s}$. They also found no significant effect of pressure on gas holdup in that range of operating variables.

The main aim of this study is to investigate the effect of pressure on gas holdup over a broader range of superficial gas velocities using a non-intrusive technique, Computed Tomography (CT). Since the effect of column diameter on holdup profiles is still debated when using small diameter columns, holdup data was to be collected in a 6" diameter column which was constructed especially for this purpose at CREL-WU. A gamma ray based computer tomography system (CT) was developed (Kumar et al., 1995) for imaging phase holdup distribution in two-phase flow systems such as bubble columns and fluidized beds. The CT measurements were performed using an encapsulated $\gamma$-ray radiation source (Cs-137) and a fan beam arrangement of detectors. The details of the hardware and software have been described elsewhere (Kumar, 1994; Kumar et al., 1995, 1997) and will not be repeated here. Instead, the obtained experimental measurements for time averaged holdup cross-sectional distributions are discussed. The average cross-sectional holdup is compared to values obtained from various correlations. 


\subsection{The High Pressure Experimental Facility}

Figure 3.1 displays the flowsheet for the high pressure system used in this study. The system is designed to handle a high flow rate of air up to $5000 \mathrm{SCFH}$ at a pressure of up to 175 psig. All the equipment is designed to support 200 psig. The bubble column is made of a stainless steel tube with inner diameter $0.169 \mathrm{~m} \mathrm{(6.359")}$, and height $2.5 \mathrm{~m}(8.2$ $\mathrm{ft})$. Figure 3.2 shows the sketch of the column including the bottom flange which has been modified to serve as a support as well. Figure 3.3 shows the sketch of the elevation of the column along the section AA as indicated on the schematic of Figure 3.2.

As shown in Figure 3.3, a transparent glass window is situated at the top of the column and is named "blue eye". This window allows viewing the system before starting the CT scan. The gas was dispersed into the column through a perforated plate distributor. The distributor, shown schematically in Figure 3.4, has 61 holes each of $0.4 \mathrm{~mm}$ diameter, providing an open area of $0.05 \%$. The holes are arranged in 3 concentric circles with 20 holes on each circle and one at the center of the distributor plate. The increment in radius between the circles on which holes are centered is $1.5 \mathrm{~cm}$. This particular distributor was used because a significant amount of data for holdup distribution and liquid velocity is available for it from our previous studies. Table 3.1 lists the operating conditions used in this study.

Air was used as the gas phase. As the liquid phase, tap water $(\sigma \mathrm{L}=72 \mathrm{mN} / \mathrm{m}, \mu \mathrm{L}=993$ $\mu$ Pa.s) was used. The experiments were conducted batchwise with respect to the liquid, but with a continuous flow of gas at ambient temperature $\left(T=25^{\circ} \mathrm{C}\right)$. Air was filtered before being introduced into the system. The gas flow rate was controlled by the rotameters at the desired values. After exiting the bubble column, the gas passes through a back-pressure regulator, which is used to control the pressure in the column. It is then discharged into the atmosphere through the vent. Two pressure safety valves are mounted both at the top and bottom of the column to prevent accidental over-pressurization. 


\section{$\rightarrow \infty \quad$ Ball or Gate \\ It Metering (Needle) valves}

Rotameter

$1 \quad 0-100 \quad \mathrm{SCFH}$

$20-500 \mathrm{SCFH}$

$3 \quad 0-2000 \mathrm{SCFH}$

$4 \quad 0-5000 \mathrm{SCFH}$

PI Pressure Indicator

PT Pressure Transducer

TI Temperature Indicator

PSV Pressure Safety Valve

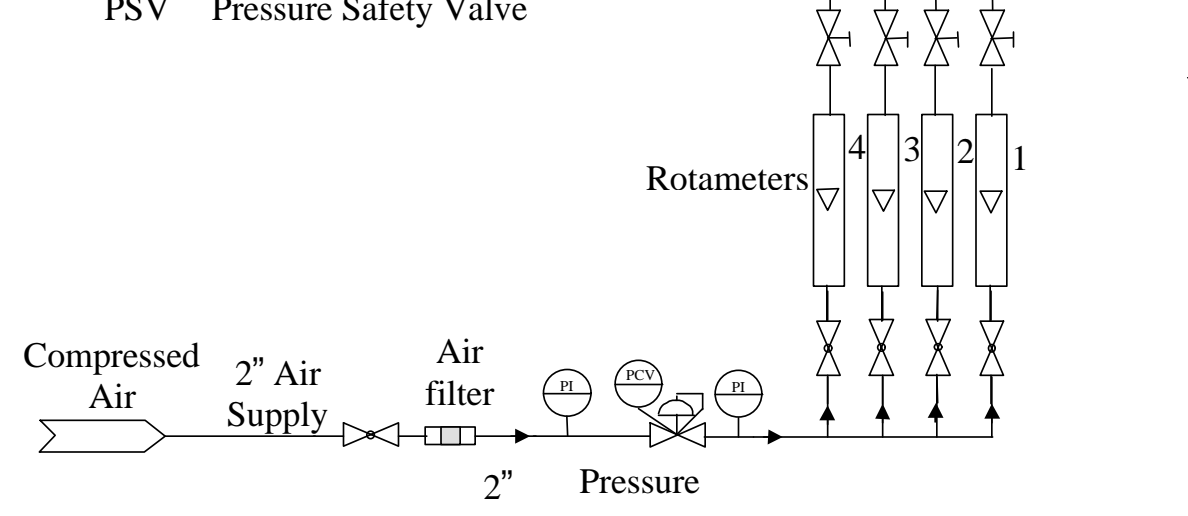

Figure 3.1 : Flowsheet for High Pressure Column

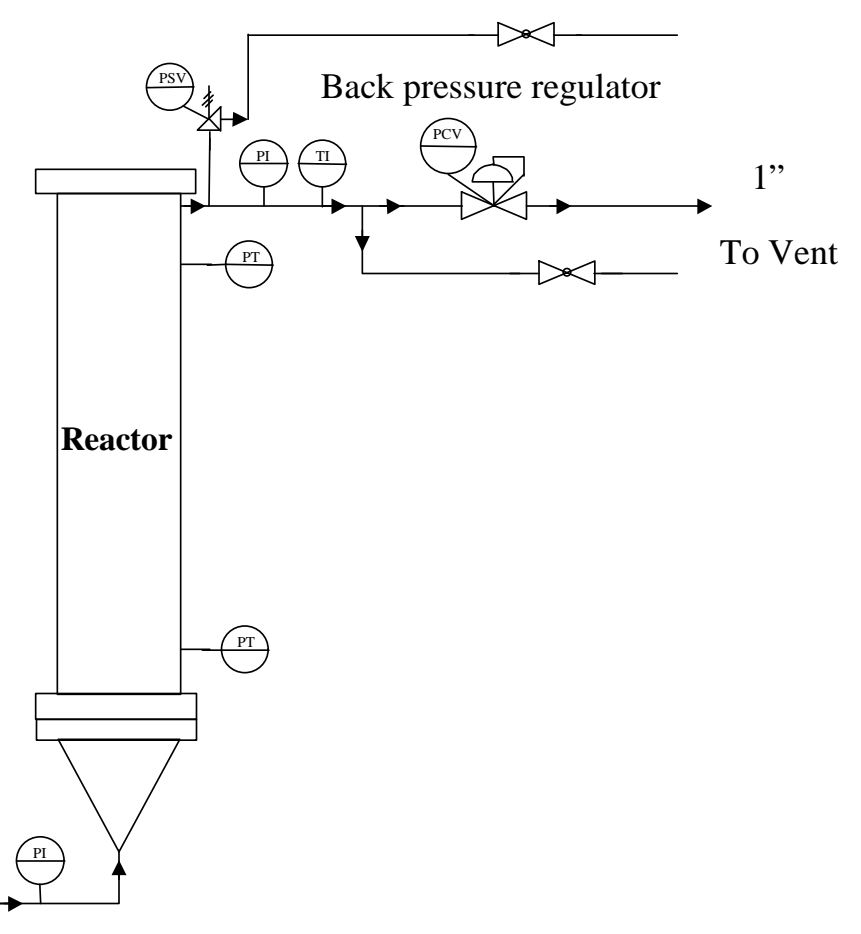




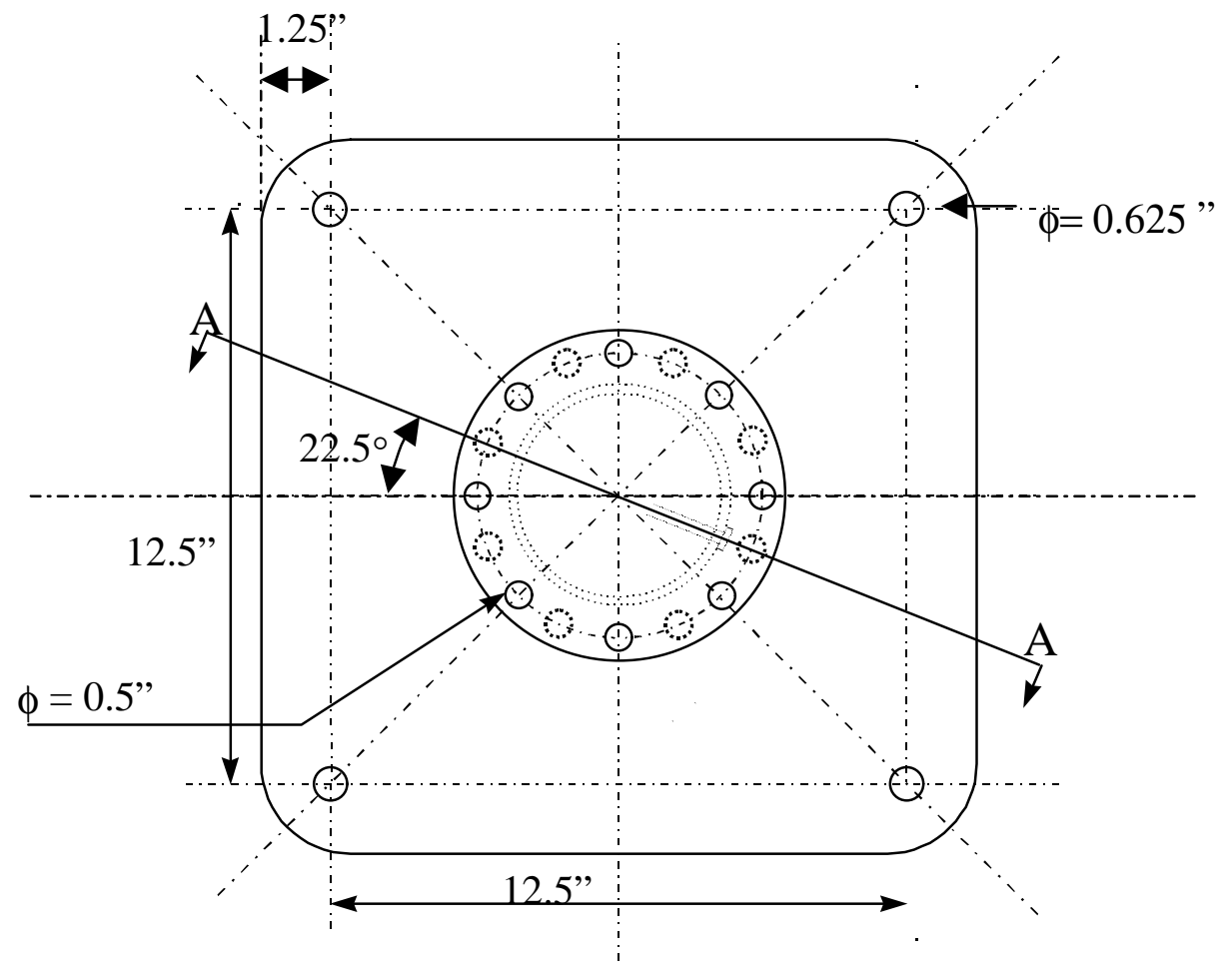

Figure 3.2: Top View of the High Pressure Column 


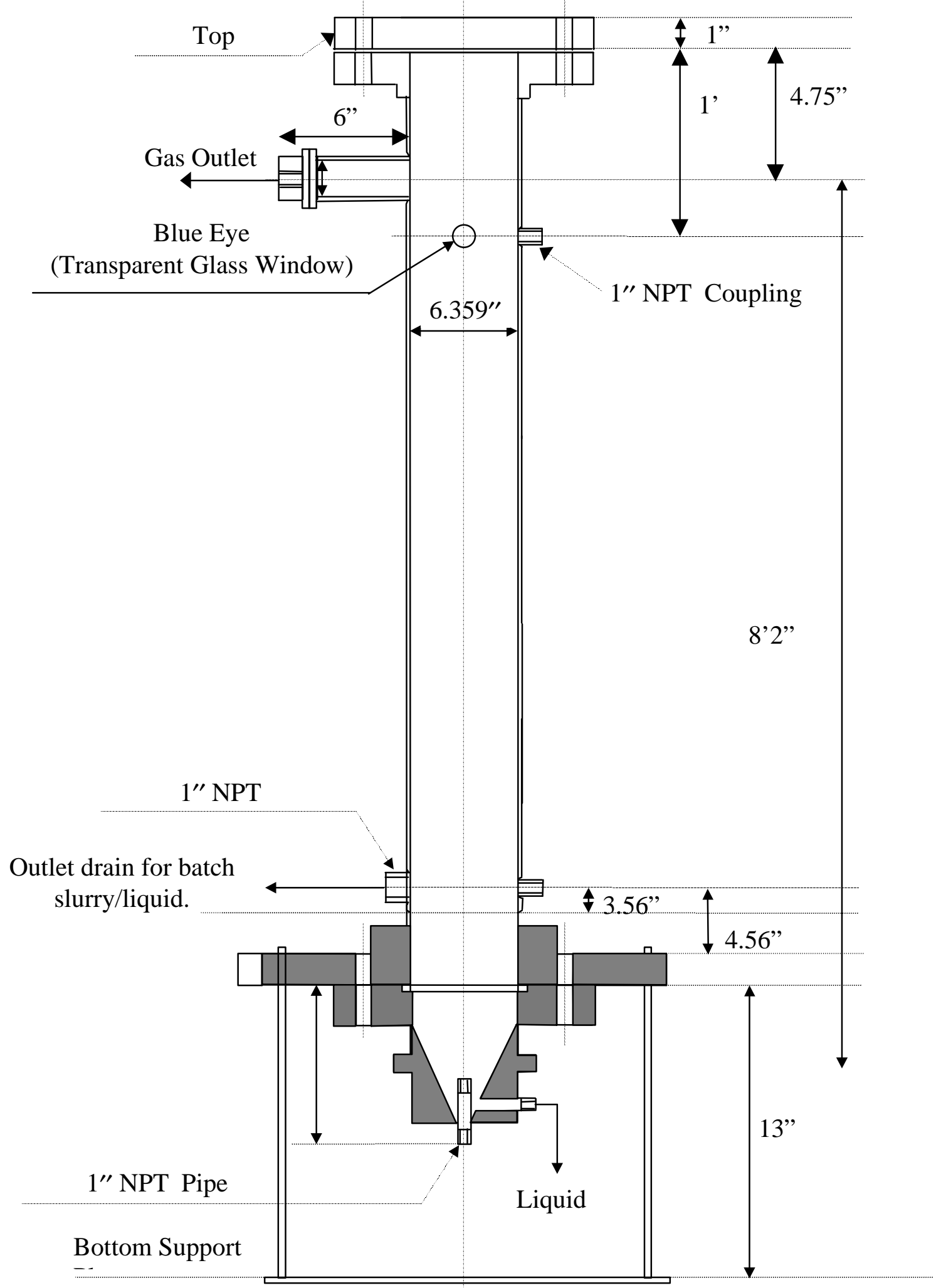

Figure 3.3: $\quad$ Side View of the High Pressure Bubble Column Elevation Along Section AA of Figure 3.2 


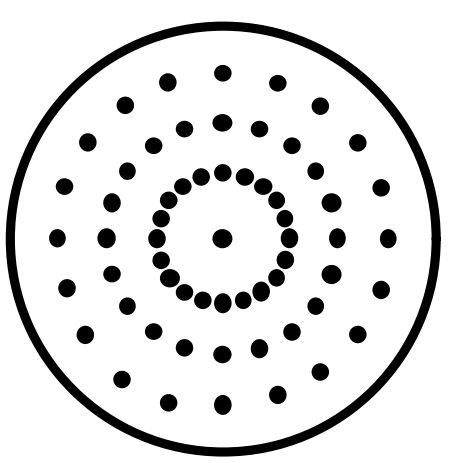

Figure 3.4: Perforated Plate Distributor of 0.04\% Open Area

Table 3.1: Operating Conditions for the High Pressure System

Diameter of column, in. $(\mathrm{cm})$

Distributor

Superficial gas velocity, $\mathrm{cm} / \mathrm{s}$

Liquid (water)

Pressure, $\mathrm{MPa}(\mathrm{atm})$

Temperature, ${ }^{\circ} \mathrm{C}$
$6.359(16.15)$

Perforated plate with 61 holes (each of $0.4 \mathrm{~mm}$ diameter), open area $0.05 \%$ (Fig. 3.4)

$2,5,12,18$

Batch

$0.1(1), 0.3(3), 0.7$ (7)

25

As already mentioned in this study the gas holdup cross-sectional distribution was measured using the gamma ray scanner and the associated tomography reconstruction algorithms developed in CREL and discussed by Kumar et al. (1995; 1997). The CREL scanner is a versatile instrument that enables the quantification of the time-averaged holdup distribution for two-phase flows under a wide range of operating conditions. The fan beam configuration of the scanner consists of an array of NaI detectors of $5 \mathrm{~cm}$ in diameter ( 5 detectors were used in this study), and an encapsulated $100 \mathrm{mCi} \mathrm{Cs}^{137}$ source located opposite to the center of the array of detectors. The measurements can be performed at different axial elevations. In this study, gas holdup distribution was measured at about 3 feet from the distributor where the flow is fully developed..

\subsection{Results and Discussion}

\subsubsection{Radial Gas Holdup Distribution}

Figures 3.5 and 3.6 display the radial gas holdup distribution at different operating pressures each at a different superficial gas velocity. These plots were obtained by azimuthally averaging the CT measured holdup distribution in the cross-section of the column 3 feet above the distributor. Our past experience indicates that at L/D ratio of 6 or above, the holdup profile is well-developed and relatively invariant to axial position 
(Kumar, 1994). From these figures, it can be seen that the differences in holdup profiles due to pressure increase with increasing superficial gas velocity.

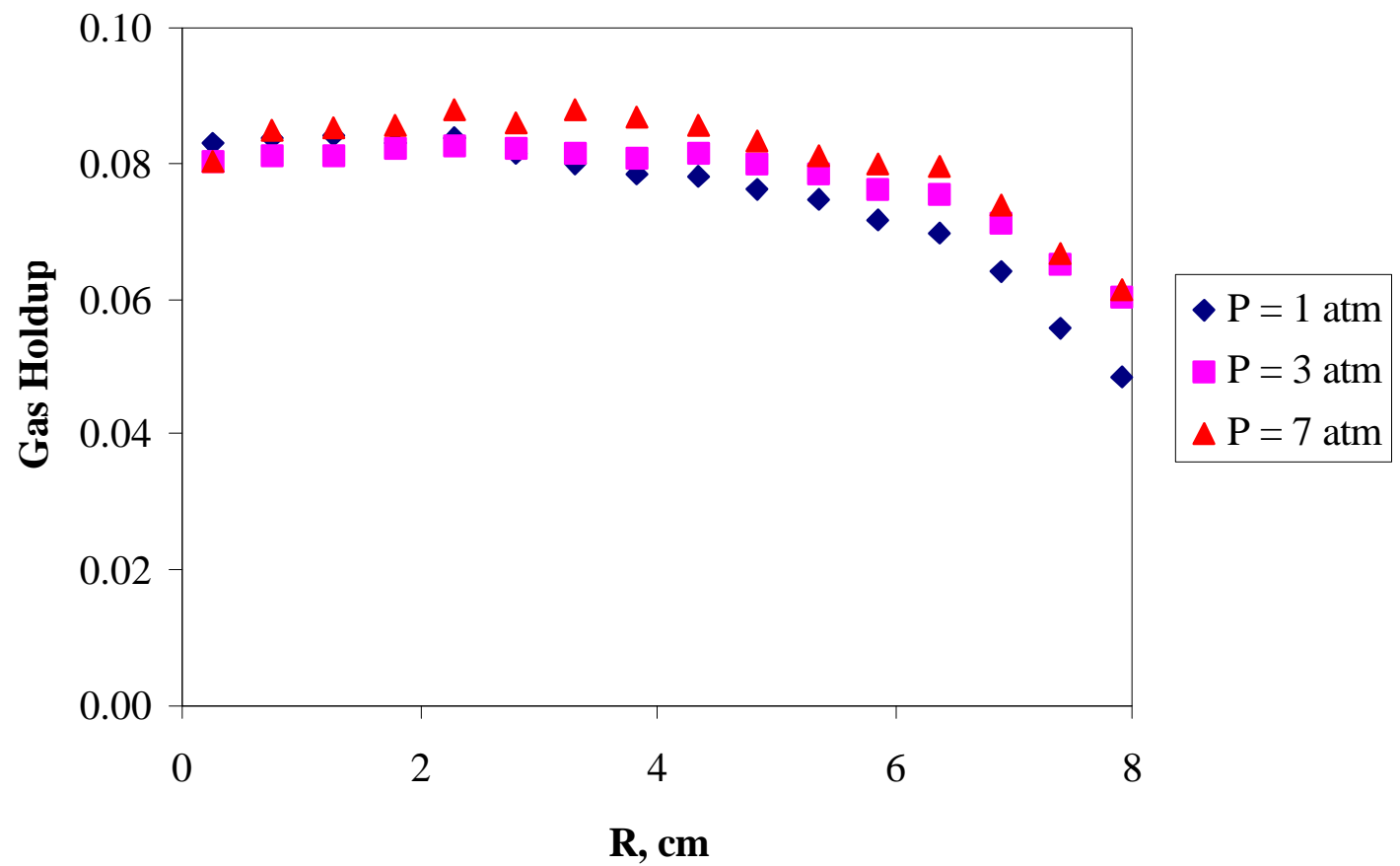

Figure 3.5: Radial Gas Holdup Distribution as a Function of Pressure for $U_{g}=2 \mathrm{~cm} / \mathrm{s}$

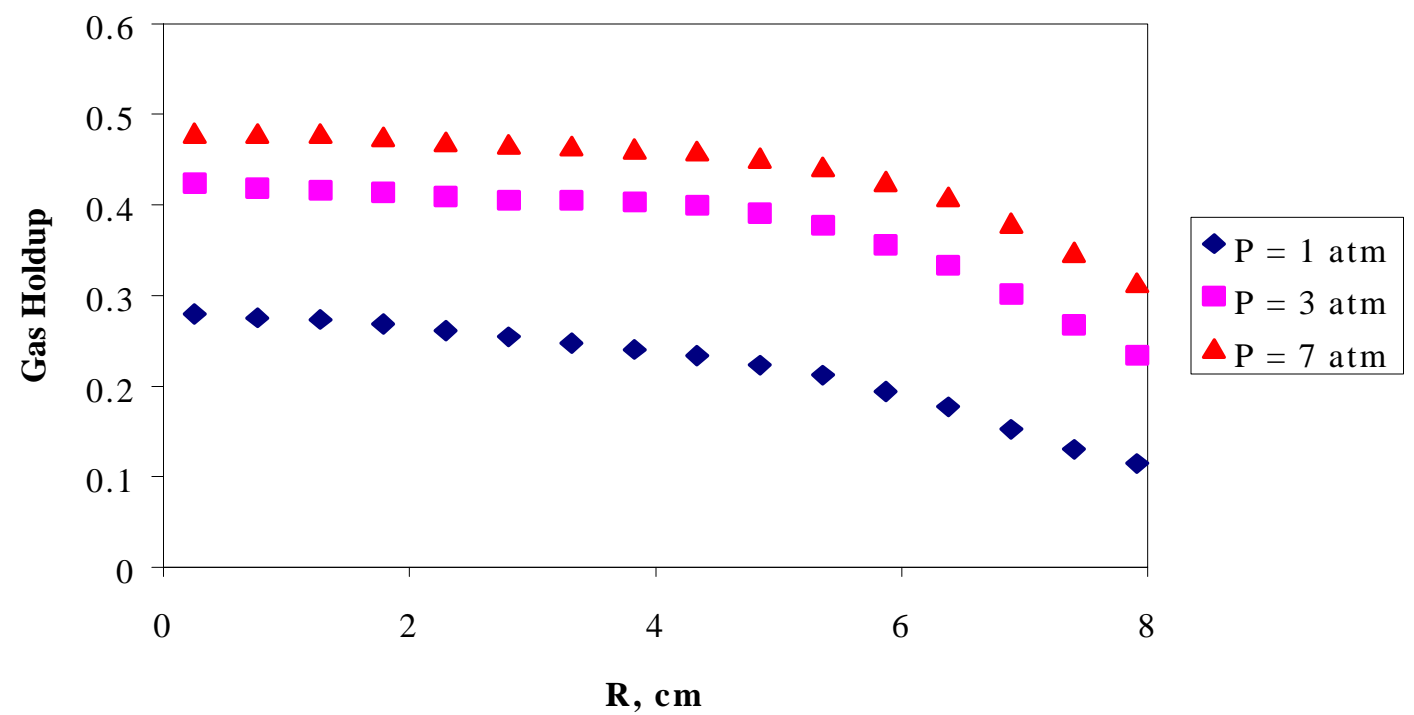

Figure 3.6: Radial Gas Holdup Distribution as a Function of Pressure for $U_{g}=12 \mathrm{~cm} / \mathrm{s}$ 
Figure 3.7 displays the results obtained by Shollenberger et al. (1995) at atmospheric conditions. The trend in gas holdup at atmospheric pressure with increasing superficial gas velocities observed by us agrees well with their results. However, the gas holdup obtained in this study is slightly higher than that from Shollenberger et al. For example, at a superficial gas velocity of $11.76 \mathrm{~cm} / \mathrm{s}$, the gas holdup value at the center, reported by Shollenberger et al., is about 0.205 (Figure 3.7), whereas in our case, a center line gas holdup value of about 0.28 is obtained at superficial gas velocity of $12 \mathrm{~cm} / \mathrm{s}$ (Figure 3.6). The discrepancies might be due to the type of distributor used or quality of water. Shollenberger $e t$ al. used a bubble cap distributor, whereas in this study a perforated plate with more holes in the center, which might enhance a non-uniform distribution, was used.

From Figures 3.5 and 3.6, it is evident that gas holdup increases both with pressure, and with superficial gas velocity in agreement with the reports in the literature (Idogawa et al., 1986, Jiang et al., 1995, Kojima et al., 1991, Kojima et al., 1987, Lin et al. (1998), and Oyevaar et al., 1989). At a velocity of $12 \mathrm{~cm} / \mathrm{s}$ (Figure 3.6), the radial gas holdup profile at atmospheric pressure is distinctly parabolic in nature, indicating churn turbulent flow, whereas at higher pressure, the profile is flatter. However, Shollenberger et al. (1996; 1997), who used a different gamma tomography system, report somewhat different observations. This can be seen in Figure 3.8, which displays the radial gas holdup distribution at $10 \mathrm{~cm} / \mathrm{s}$ superficial gas velocity in of a $0.48 \mathrm{~m}$ ID and $3 \mathrm{~m}$ tall column, where they found that the gas holdup profile is parabolic at a pressure of $0.394 \mathrm{MPa}$. In their study, the sparger was a $15 \mathrm{~cm}$ diameter ring formed from 1.1-cm ID stainless steel tubing. There were 12 holes equidistantly distributed on the ring, each of $3.18 \mathrm{~mm}$ in diameter. Hence, the discrepancies might be due also to the type of distributor and size of column used. The exact cause is not known at present.

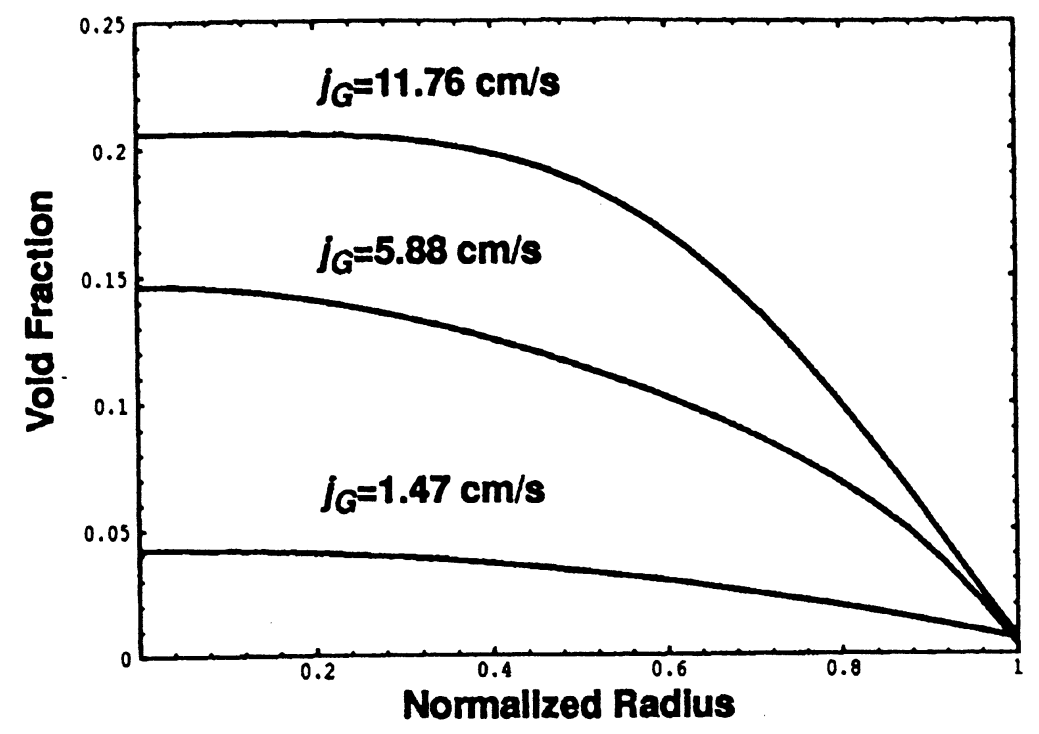

Figure 3.7: Radial Distribution of Gas Holdup at Atmospheric Conditions as a Function of Superficial Gas Velocity for $19 \mathrm{~cm}$ Air-Water Column with Bubble Cap Distributor (Shollenberger et al., 1995) 


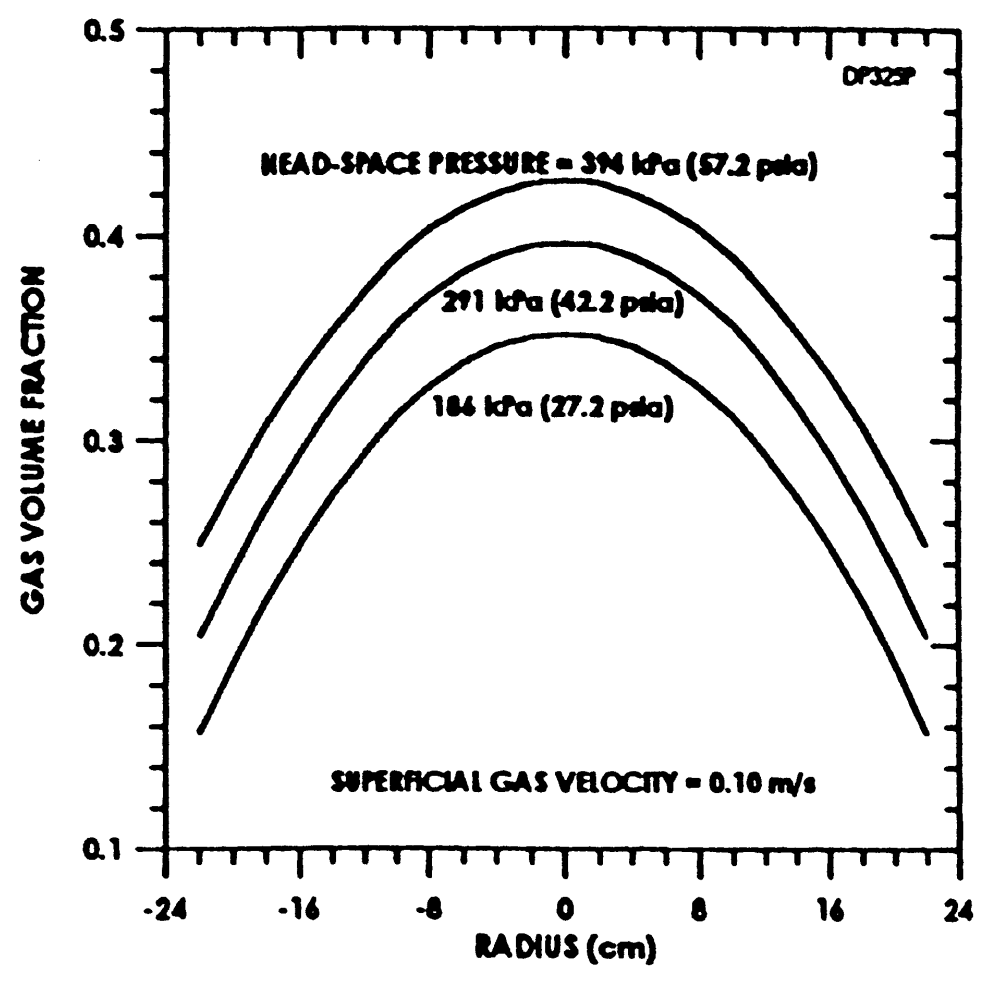

Figure 3.8: Radial Distribution of Gas Holdup as a Function of Pressure for Ug $=10$ $\mathrm{cm} / \mathrm{s}$ (Taken from Shollenberger et al., 1996)

Figures 3.9 and 3.10 display the cross-sectional distribution of the gas holdup for the bubble column at different pressures for two superficial gas velocity studied. For example, Figure 3.9 (a) shows the pixel map for $\mathrm{P}=1 \mathrm{~atm}$, and $\mathrm{U}_{\mathrm{g}}=2 \mathrm{~cm} / \mathrm{s}$. The map indicates that gas holdup at these conditions has a maximum value of 0.1 , and this occurs near the center of the column, which shows a darker area. With this interpretation, one can see that as the gas superficial velocity and column pressure increase, gas holdup increases (Figure 3.10 (d)).

Thus, a gradual variation in the color shades for the gas holdup from the column center to the wall indicates a change in gas holdup value. These plots confirm that gas holdup increases with pressure and superficial gas velocities. Visual observations of the column in the vicinity of the wall via the "blue eye" revealed much smaller bubbles when pressure is increased. The coalescence rate seems to be decreased and bubble breakup seems promoted at pressurized conditions. Hence, gas holdup increases as pressure increases.

\subsubsection{Cross-Sectional Average Gas Holdup}

The cross-sectional averaged gas holdup is calculated using Equation (3.1) and can be taken as a good estimate of the overall gas holdup (Kumar, 1994). 


$$
\bar{\varepsilon}_{\mathrm{g}}=\frac{2}{\mathrm{R}^{2}} \int_{0}^{\mathrm{R}} \mathrm{r} \varepsilon(\mathrm{r}) \mathrm{dr}
$$

Figure 3.11 displays the cross-sectional average gas holdup as a function of superficial gas velocity at various pressures, while Figure 3.12 displays the given data for the crosssectional average gas holdup as a function of pressure at different superficial gas velocities. Table 3.2 lists the calculated cross-sectional average gas holdup values for different operating conditions.

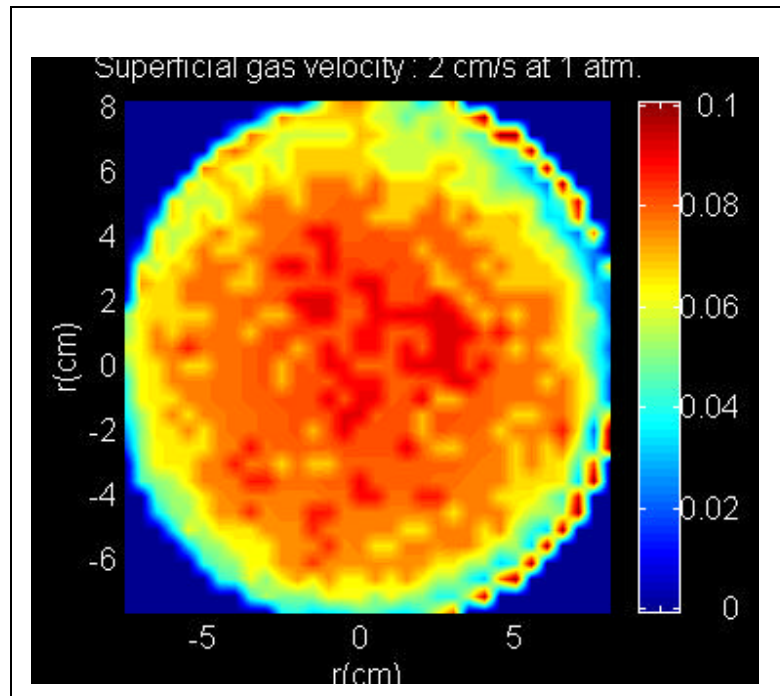

(a)

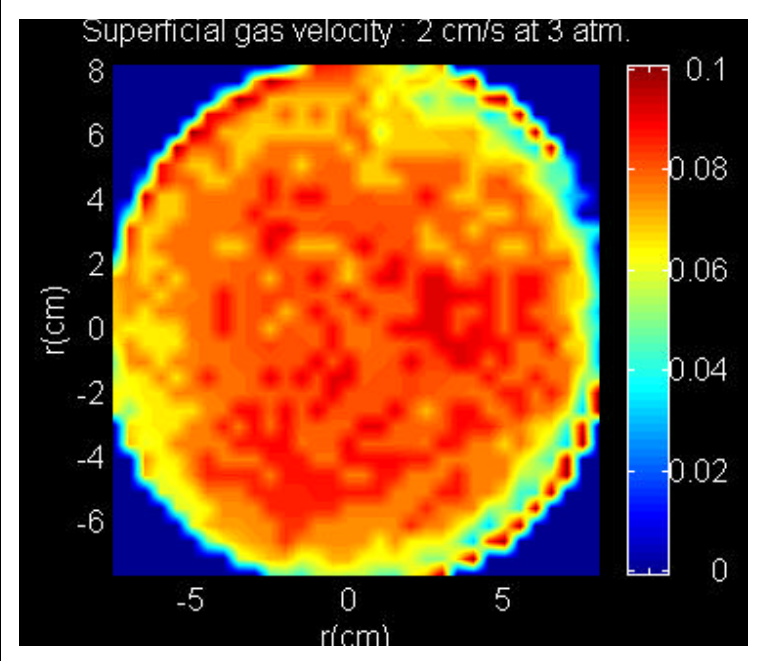

(b)

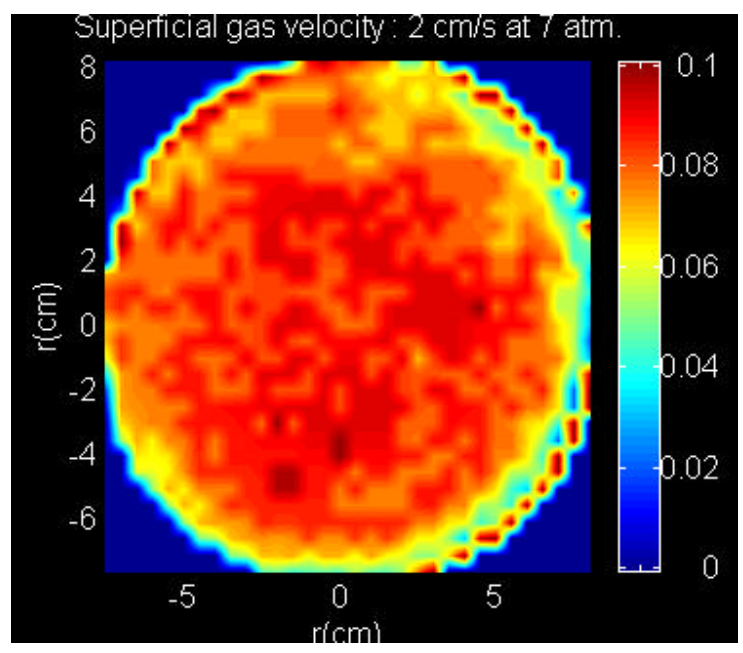

(c)

Figure 3.9: Cross-sectional Distribution of the Gas Holdup at (a) $\mathrm{P}=1$ atm, (b) $\mathrm{P}=3$ atm, and (c) $\mathrm{P}=7$ atm for $\mathrm{U}_{\mathrm{g}}=2 \mathrm{~cm} / \mathrm{s}$ 


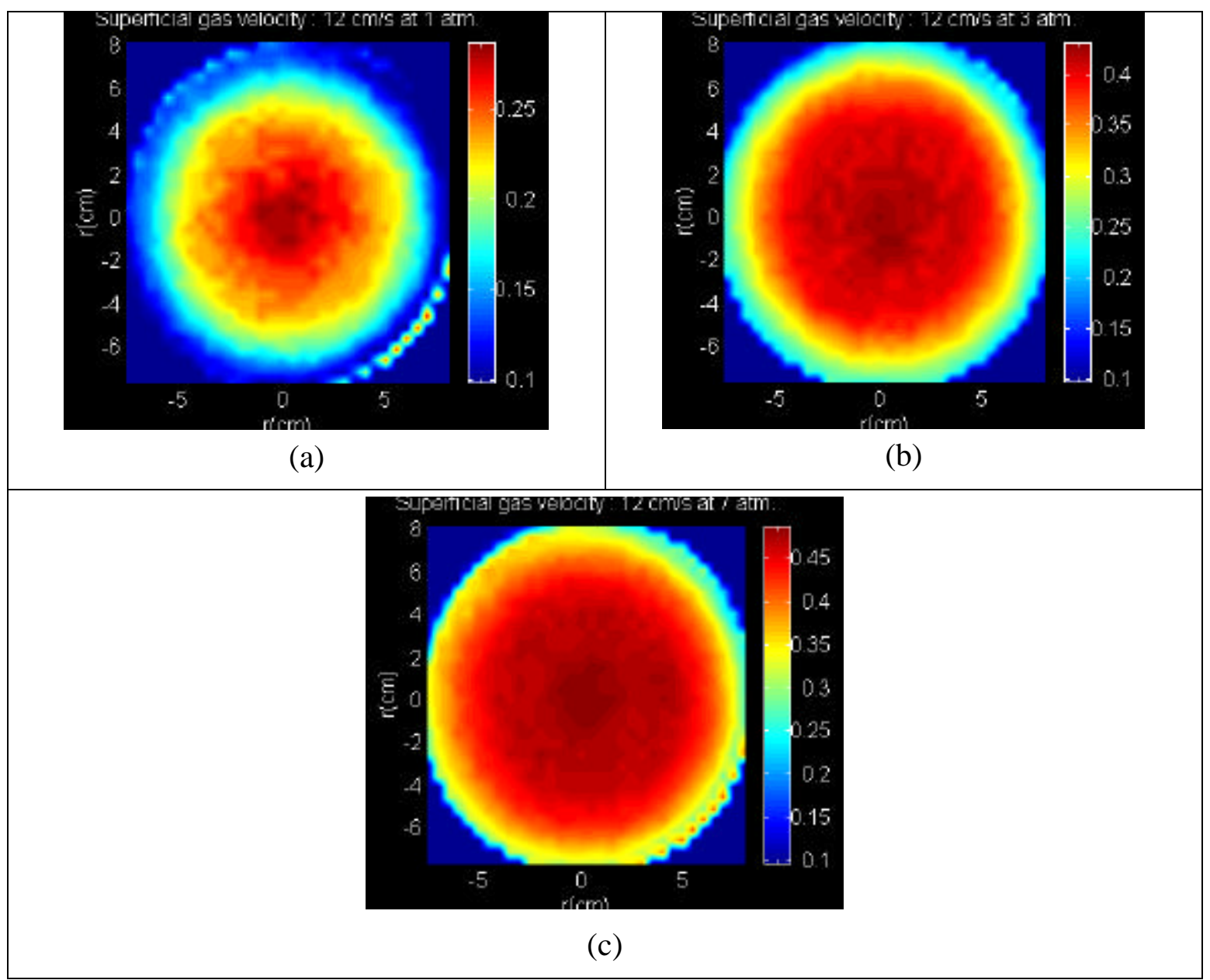

Figure 3.10: Cross-sectional Distribution of the Gas Holdup at (a) $\mathrm{P}=1$ atm, (b) $\mathrm{P}=3$ atm, and (c) $\mathrm{P}=7$ atm for $\mathrm{U}_{\mathrm{g}}=12 \mathrm{~cm} / \mathrm{s}$

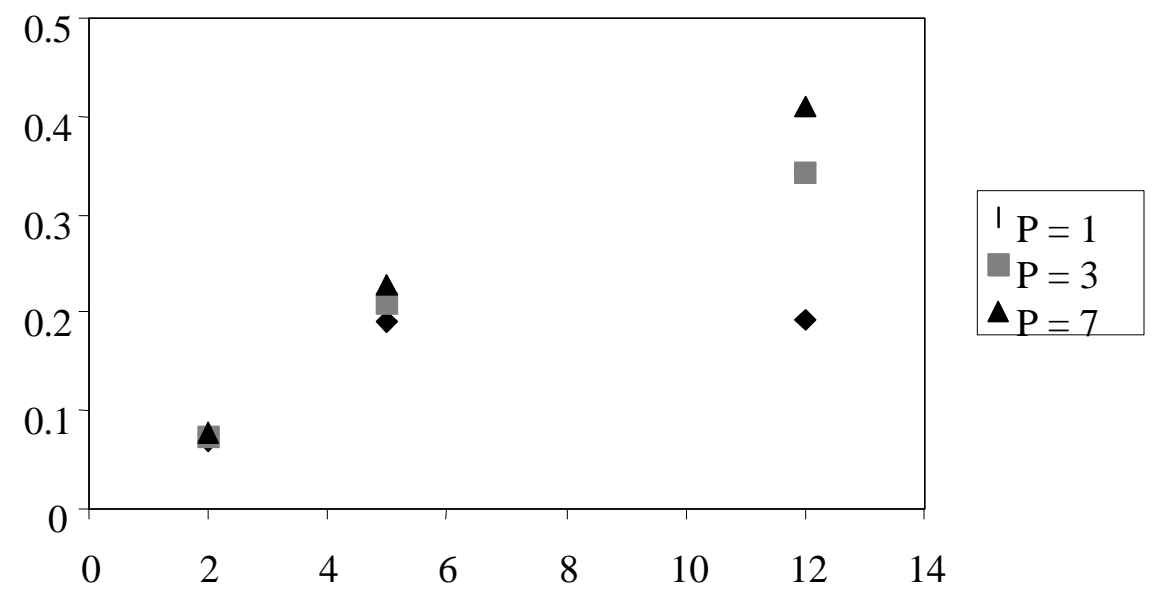

Superficial Gas Velocity, $\mathrm{cm} / \mathrm{s}$

Figure 3.11: Cross-sectional Average Gas Holdup as a Function of Superficial Gas Velocity at Different Pressures 


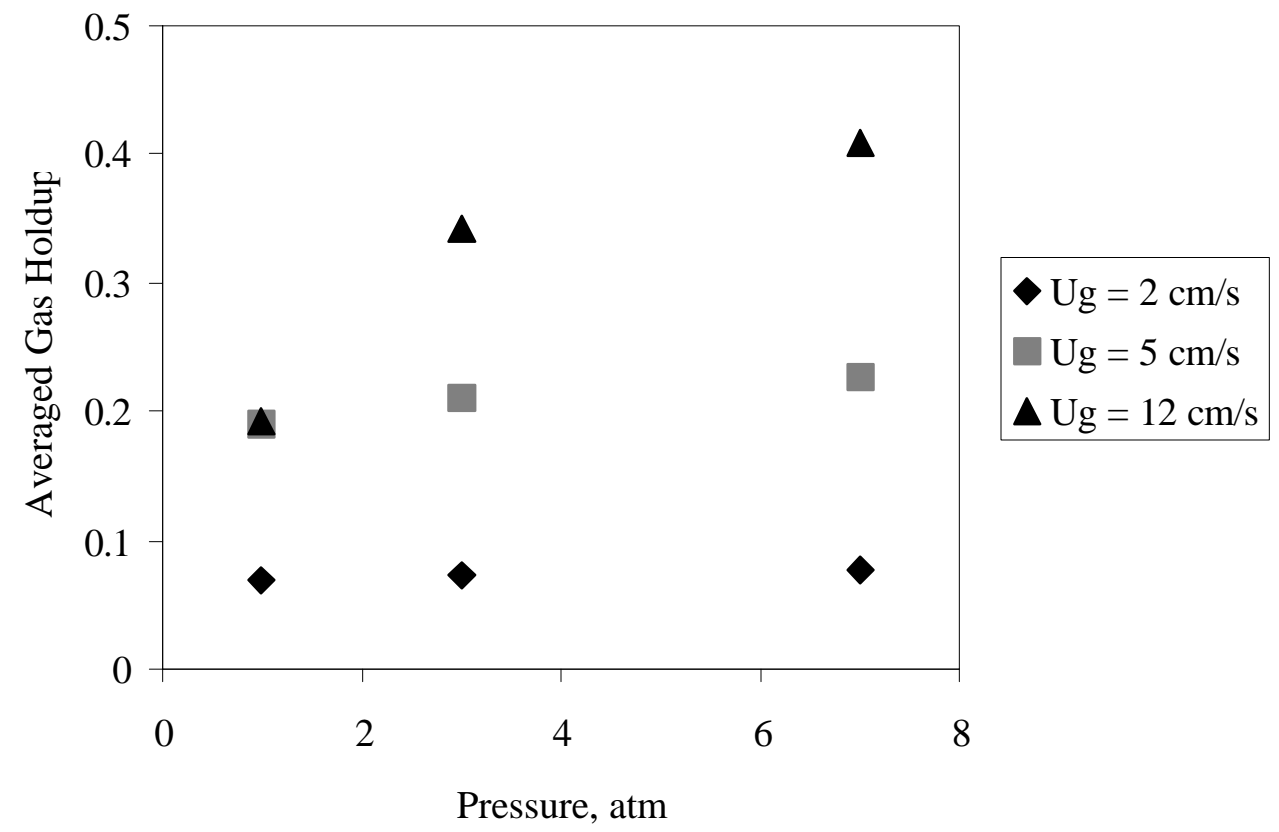

Figure 3.12: Cross-sectional Averaged Gas Holdup as a Function of Pressure at Different Superficial Gas Velocities

Table 3.2: $\quad$ Cross-Sectional Average Gas Holdup at Different Operating Conditions

\begin{tabular}{ccc}
\hline \hline Pressure, atm & Superficial gas velocity, $\mathrm{cm} / \mathrm{s}$ & $\bar{\varepsilon}_{g}$ \\
\hline 1 & 2 & 0.069 \\
& 5 & 0.191 \\
& 12 & 0.193 \\
\hline 3 & 2 & 0.074 \\
& 5 & 0.210 \\
& 12 & 0.342 \\
\hline 7 & 2 & 0.077 \\
& 5 & 0.227 \\
& 12 & 0.410 \\
\hline \hline
\end{tabular}


Figures 3.11 and 3.12 further confirm that gas holdup increases with pressure, except at low superficial gas velocities (below and up to $5 \mathrm{~cm} / \mathrm{s}$ ) when it is rather insensitive to pressure as reported in the literature (Kölbel et al., 1961; Deckwer et al., 1980). At atmospheric pressure, the cross-sectional average gas holdup seems almost constant after certain gas superficial velocity is reached as indicated by Figure 3.10. This leveling off effect seems to occur at higher gas velocities at higher pressures, as evident from Figures 3.11 and 3.12 .

\subsection{Comparison with Various Correlations in the Literature}

Numerous correlations for overall gas holdup in bubble columns have been reported and those that seem applicable to the conditions investigated in this study are summarized in Table 3.3. Since Kumar (1994) has shown that the cross-sectional average holdup measured at heights above the distributor larger than 4 to 5 column diameters is in close agreement with the overall gas holdup in the column, the cross sectional average holdup determined in this study was compared to the prediction for overall gas holdup obtained from the reported correlations. Table 3.4 lists gas holdup values obtained using the correlations shown in Table 3.3 and the error in predictions. Figure 3.13 shows the predictions for the overall gas holdup at $\mathrm{P}=1 \mathrm{~atm}$ as a function of superficial gas velocity based on various correlations. It can be concluded that none of the correlations, except Akita and Yoshida's (1973), agrees closely with the experimental data. At $U_{g}=5 \mathrm{~cm} / \mathrm{s}$, even Akita and Yoshida's prediction deviates from the observed holdup.

Since the superficial gas velocity of $5 \mathrm{~cm} / \mathrm{s}$ is close to the transition velocity at $\mathrm{P}=1 \mathrm{~atm}$ that changes bubbly flow into churn turbulent flow, and the precise value of the transition velocity is a function of the unmeasurable water quality, it is possible that the deviation between data and correlation predictions at $U_{\mathrm{g}}=5 \mathrm{~cm} / \mathrm{s}$ is caused by the fact that the correlations predict the holdup in one flow regime while the data reflects the other flow regime. It is evident from Figure 3.13 that the experimental holdup value at $U_{g}=5 \mathrm{~cm} / \mathrm{s}$ at $\mathrm{P}=1 \mathrm{~atm}$ is considerably higher than the value predicted by any of the correlations indicating perhaps a different flow regime during our experiment than observed in the data used to develop the correlations. 
Table 3.3: $\quad$ Correlations for Gas Holdup

\begin{tabular}{|c|c|c|c|c|}
\hline References & "Gas-Liquid System & Apparatus & Conditions & Correlations \\
\hline $\begin{array}{l}\text { Akita \& } \\
\text { Yoshida (1973) }\end{array}$ & $\begin{array}{l}\mathrm{He} / \mathrm{CO}_{2} / \mathrm{O}_{2} / \text { air- } \mathrm{H}_{2} \mathrm{O} / \\
\mathrm{Glycol} / \mathrm{Methanol} / \mathrm{CCl}_{4} / \\
\mathrm{Na}_{2} \mathrm{SO}_{3} / \mathrm{NaCl}\end{array}$ & $\begin{array}{l}\mathrm{D}=0.152,0.301,0.6 \mathrm{~m} \\
\text { Sparger }(5.0 \mathrm{~mm})\end{array}$ & $\begin{array}{l}\mathrm{P}=0.1 \mathrm{MPa} \\
\mathrm{T}=283-313 \mathrm{~K} \\
\mathrm{U}_{\mathrm{g}}=0.5-40 \mathrm{~cm} / \mathrm{s}\end{array}$ & $\frac{\bar{\varepsilon}}{(1-\bar{\varepsilon})^{4}}=0.2\left(\frac{\mathrm{gD}^{2} \rho_{1}}{\gamma}\right)^{0.125}\left(\frac{\mathrm{gD}^{3}}{v_{1}}\right)^{0.083} \frac{\mathrm{U}_{\mathrm{g}}}{(\mathrm{gD})^{0.5}}$ \\
\hline $\begin{array}{l}\text { Hikita et al. } \\
\text { (1981) }\end{array}$ & $\begin{array}{l}\mathrm{H}_{2} / \mathrm{CO}_{2} / \mathrm{CH}_{4} / \mathrm{C}_{3} \mathrm{H}_{8} / \mathrm{H}_{2}+\mathrm{N}_{2} / \\
\text { air- } \mathrm{H}_{2} \mathrm{O} / \mathrm{Sucrose} / \\
\text { Methanol/n-Butanol/ } \\
\text { Aniline } / \mathrm{i}-\mathrm{Butanol} / \mathrm{NaCl} / \\
\mathrm{Na}_{2} \mathrm{SO}_{4} / \mathrm{CaCl}{ }_{2} / \mathrm{MgCl}_{2} / \\
\mathrm{AlCl}_{3} / \mathrm{KCl} / \mathrm{K}_{2} \mathrm{SO}_{4} / \mathrm{K}_{3} \mathrm{PO}_{4} / \\
\mathrm{KNO}_{3}\end{array}$ & $\begin{array}{l}\mathrm{D}=0.10 \mathrm{~m} \\
\text { Nozzle }(1.1 \mathrm{~cm})\end{array}$ & $\begin{array}{l}\mathrm{P}=0.1 \mathrm{MPa} \\
\mathrm{H} / \mathrm{D}=15 \\
\mathrm{U}_{\mathrm{g}}=4.2-38 \mathrm{~cm} / \mathrm{s}\end{array}$ & $\begin{array}{l}\varepsilon=0.672 f\left(\frac{U_{g} \mu_{l}}{\gamma}\right)^{0.578}\left(\frac{\mu_{l}^{4} g}{\rho_{l} \gamma^{3}}\right)^{-0.131}\left(\frac{\rho_{g}}{\rho_{l}}\right)^{0.062}\left(\frac{\mu_{g}}{\mu_{l}}\right)^{0.107} \\
\text { where } \mathrm{f}=\left\{\begin{array}{cl}1.0 & \text { for non-electrolyte solution } \\
10^{0.0414 \mathrm{I}} & \text { for } 0<\mathrm{I}<1.0 \mathrm{~kg} \text { ion } / \mathrm{m}^{3} \\
1.1 & \text { for } \mathrm{I}>1.0 \mathrm{~kg} \text { ion } / \mathrm{m}^{3}\end{array}\right.\end{array}$ \\
\hline $\begin{array}{l}\text { Hammar et al. } \\
\text { (1984) }\end{array}$ & & & & $\frac{\bar{\varepsilon}}{1-\bar{\varepsilon}}=0.4\left(\frac{\mathrm{U}_{\mathrm{g}} \mu_{1}}{\gamma}\right)^{0.87}\left(\frac{\mu_{1}^{4} \mathrm{~g}}{\rho_{1} \gamma^{3}}\right)^{-0.27}\left(\frac{\rho_{\mathrm{g}}}{\rho_{1}}\right)^{0.17}$ \\
\hline $\begin{array}{l}\text { Idogawa et al. } \\
(1985)\end{array}$ & Air- $\mathrm{H}_{2} \mathrm{O}$ & $\begin{array}{l}\mathrm{D}=0.05 \mathrm{~m} \\
\text { Porous plate }(2,100 \mu \mathrm{m}) \\
\text { Capillary tubes }(1,3,5 \mathrm{~mm}) \\
\text { Perforated plate }(19 \text { holes of } 1 \mathrm{~mm})\end{array}$ & $\begin{array}{l}\mathrm{P}=0.1-15 \mathrm{MPa} \\
\mathrm{T}=288-293 \mathrm{~K} \\
\mathrm{H} / \mathrm{D}=16.6 \\
\mathrm{U}_{\mathrm{g}}=0.5-5 \mathrm{~cm} / \mathrm{s}\end{array}$ & $\frac{\bar{\varepsilon}}{1-\bar{\varepsilon}}=1.44 \mathrm{U}_{\mathrm{g}}^{0.58} \rho_{\mathrm{g}}^{0.12} \sigma_{1}^{-0.16 \exp (-\mathrm{P})}$ \\
\hline $\begin{array}{l}\text { Reilly et al. } \\
\text { (1986) }\end{array}$ & $\begin{array}{l}\mathrm{He} / \mathrm{Ar} / \mathrm{air}-\mathrm{H}_{2} \mathrm{O} / \text { solvent/ } \\
\text { trichloroethylene-glass } \\
\text { beads }\end{array}$ & $\begin{array}{l}\mathrm{D}=0.3 \mathrm{~m} \\
\text { Perforated plate }(293 \text { holes }, 1.5 \mathrm{~mm}) \\
\text { Single sparger } \\
\text { Multiorifice sparger }(13.4 \mathrm{~mm})\end{array}$ & $\begin{array}{l}\mathrm{P}=0.1 \mathrm{MPa} \\
\mathrm{T}=283-323 \mathrm{~K} \\
\mathrm{U}_{\mathrm{g}}=0.4-40 \mathrm{~cm} / \mathrm{s}\end{array}$ & $\bar{\varepsilon}=296 \mathrm{U}_{\mathrm{g}}^{0.44} \rho_{1}^{-0.16} \rho_{\mathrm{g}}^{0.19}+0.009$ \\
\hline $\begin{array}{l}\text { Idogawa et al. } \\
\text { (1987) }\end{array}$ & $\begin{array}{l}\mathrm{H}_{2} / \mathrm{He} / \text { Air- } \mathrm{H}_{2} \mathrm{O} / \mathrm{Methanol} / \\
\text { Ethanol/Acetone/Aqueous } \\
\text { alcohol solution }\end{array}$ & $\begin{array}{l}\mathrm{D}=0.05 \mathrm{~m} \\
\text { Perforated plate }(19 \text { holes of } 1 \mathrm{~mm})\end{array}$ & $\begin{array}{l}\mathrm{P}=0.1-5 \mathrm{MPa} \\
\mathrm{T}=284-293 \mathrm{~K} \\
\mathrm{H} / \mathrm{D}=16.6 \\
\mathrm{U}_{\mathrm{g}}=0.5-5 \mathrm{~cm} / \mathrm{s}\end{array}$ & $\frac{\bar{\varepsilon}}{1-\bar{\varepsilon}}=0.059 \mathrm{U}_{\mathrm{g}}^{0.8} \rho_{\mathrm{g}}^{0.17}\left(\frac{\sigma_{1}}{72}\right)^{-0.22 \exp (-\mathrm{P})}$ \\
\hline
\end{tabular}


Table 3.3: $\quad$ Correlations for Gas Holdup (Continued)

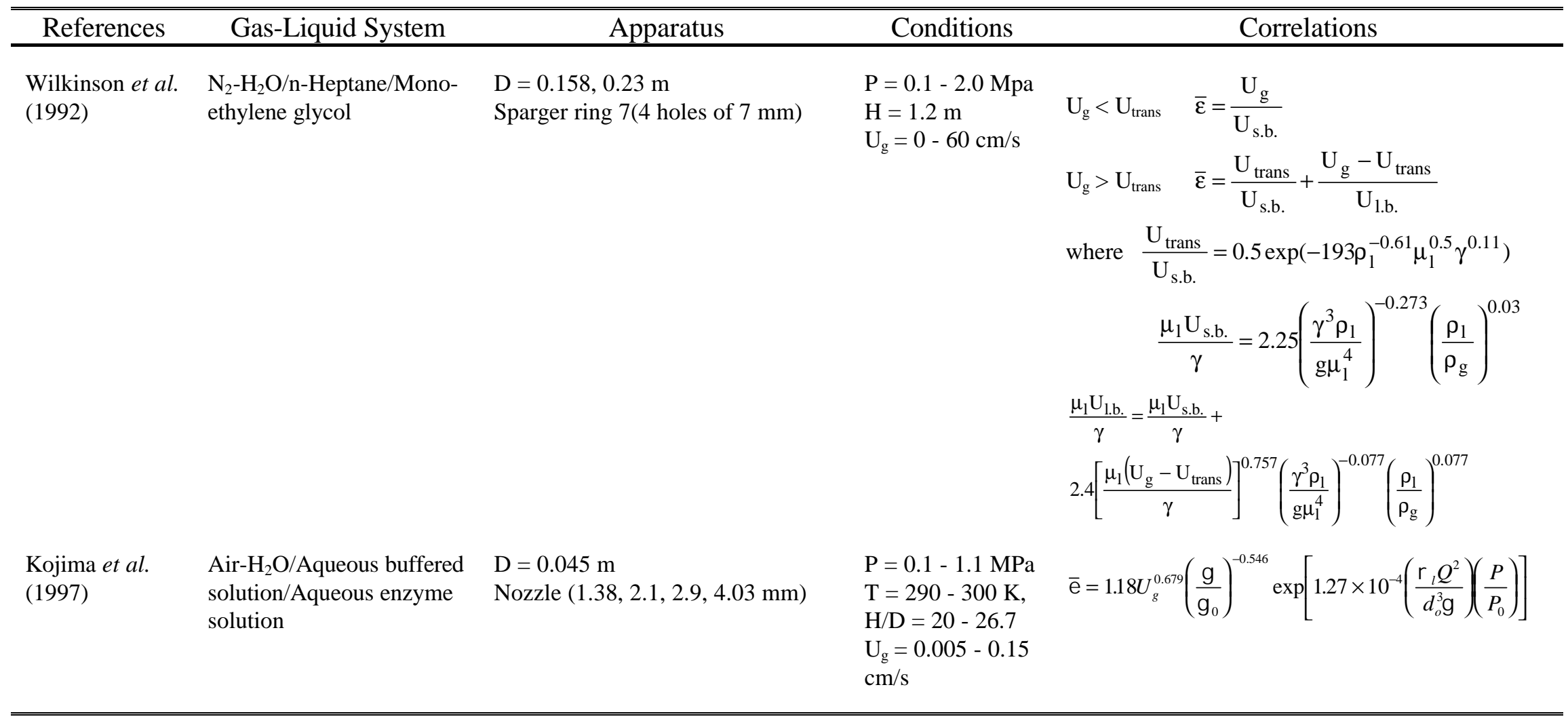


Table 3.4: Comparison of Cross-Sectional Average Gas Holdup with Predictions of Different Correlations (and Percent Error in Predictions)

$$
\text { Error }=\frac{\text { measured value }- \text { predicted value }}{\text { measure value }} \times 100
$$

\begin{tabular}{|c|c|c|c|c|c|c|c|c|c|}
\hline $\mathrm{U}_{\mathrm{g}}, \mathrm{cm} / \mathrm{s}$ & Expt'l data & $\begin{array}{c}\text { Akita } \\
(1973) \\
\end{array}$ & $\begin{array}{r}\text { Hikita } \\
(1980)\end{array}$ & $\begin{array}{c}\text { Hammer } \\
(1984)\end{array}$ & $\begin{array}{c}\text { Idogawa } \\
(1985) \\
\end{array}$ & $\begin{array}{l}\text { Reilly } \\
(1986)\end{array}$ & $\begin{array}{c}\text { Idogawa } \\
(1987) \\
\end{array}$ & $\begin{array}{c}\text { Wilkinson } \\
(1992)\end{array}$ & $\begin{array}{c}\text { Kojima } \\
(1997)\end{array}$ \\
\hline 2 & 0.069 & $0.063(8.7)$ & $0.050(28)$ & $0.040(42)$ & $0.052(25)$ & $0.059(14)$ & $0.056(19)$ & $0.050(28)$ & $0.083(20)$ \\
\hline 5 & 0.191 & $0.106(44)$ & $0.084(56)$ & $0.084(56)$ & $0.085(56)$ & $0.084(56)$ & $0.110(42)$ & $0.096(50)$ & 0.155 (19) \\
\hline 12 & 0.193 & $0.181(6.2)$ & $0.140(28)$ & $0.165(14)$ & $0.134(31)$ & $0.120(38)$ & $0.200(3.6)$ & $0.162(16)$ & $0.286(47)$ \\
\hline
\end{tabular}

\begin{tabular}{|c|c|c|c|c|c|c|c|c|}
\hline $\mathrm{U}_{\mathrm{g}}, \mathrm{cm} / \mathrm{s}$ & Expt'l data & $\begin{array}{l}\text { Hikita } \\
(1980) \\
\end{array}$ & $\begin{array}{c}\text { Hammer } \\
(1984) \\
\end{array}$ & $\begin{array}{c}\text { Idogawa } \\
(1985) \\
\end{array}$ & $\begin{array}{c}\text { Reilly } \\
(1986) \\
\end{array}$ & $\begin{array}{c}\text { Idogawa } \\
(1987) \\
\end{array}$ & $\begin{array}{c}\text { Wilkinson } \\
(1992) \\
\end{array}$ & $\begin{array}{c}\text { Kojima } \\
\text { (1997) } \\
\end{array}$ \\
\hline 2 & 0.077 & $0.056(27)$ & $0.055(29)$ & $0.084(9.1)$ & $0.081(5.2)$ & $0.077(0.0)$ & $0.054(30)$ & $0.083(7.8)$ \\
\hline 5 & 0.227 & $0.103(55)$ & $0.114(50)$ & $0.135(40)$ & $0.117(48)$ & $0.147(35)$ & $0.106(53)$ & $0.159(30)$ \\
\hline 12 & 0.410 & $0.158(62)$ & $0.215(48)$ & $0.206(50)$ & $0.168(59)$ & $0.258(37)$ & $0.181(56)$ & $0.326(20)$ \\
\hline
\end{tabular}




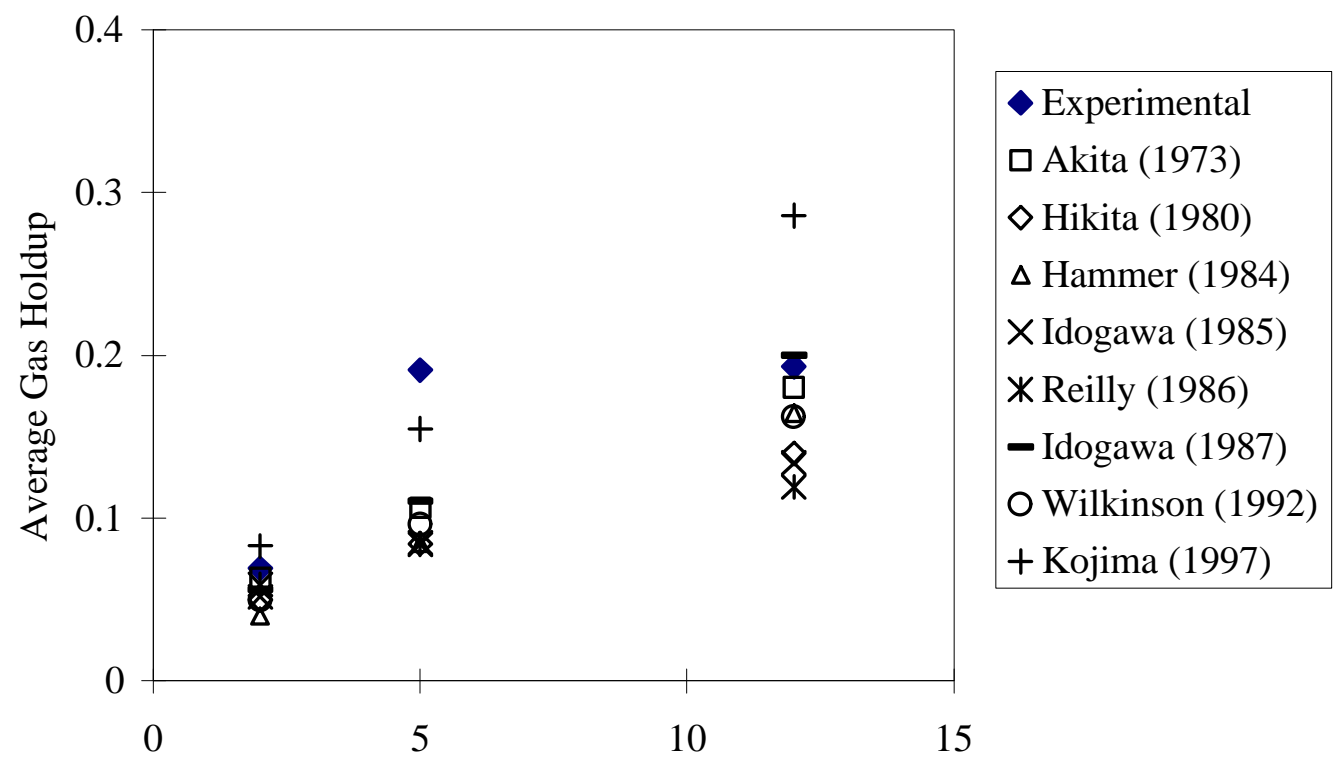

Superficial Gas Velocity, cm/s

Figure 3.13: Cross-sectional Average Gas Holdup as a Function of Superficial Gas Velocity at Atmospheric Pressure

Figure 3.14 shows the predictions for the overall gas holdup as a function $\mathrm{f}$ superficial gas velocity at $\mathrm{P}=7 \mathrm{~atm}$ and compares these predictions to our data. The numerical comparison is shown in Table 3.4. At elevated pressure the correlation of Kojima et al., 1997 comes the closest to the data.

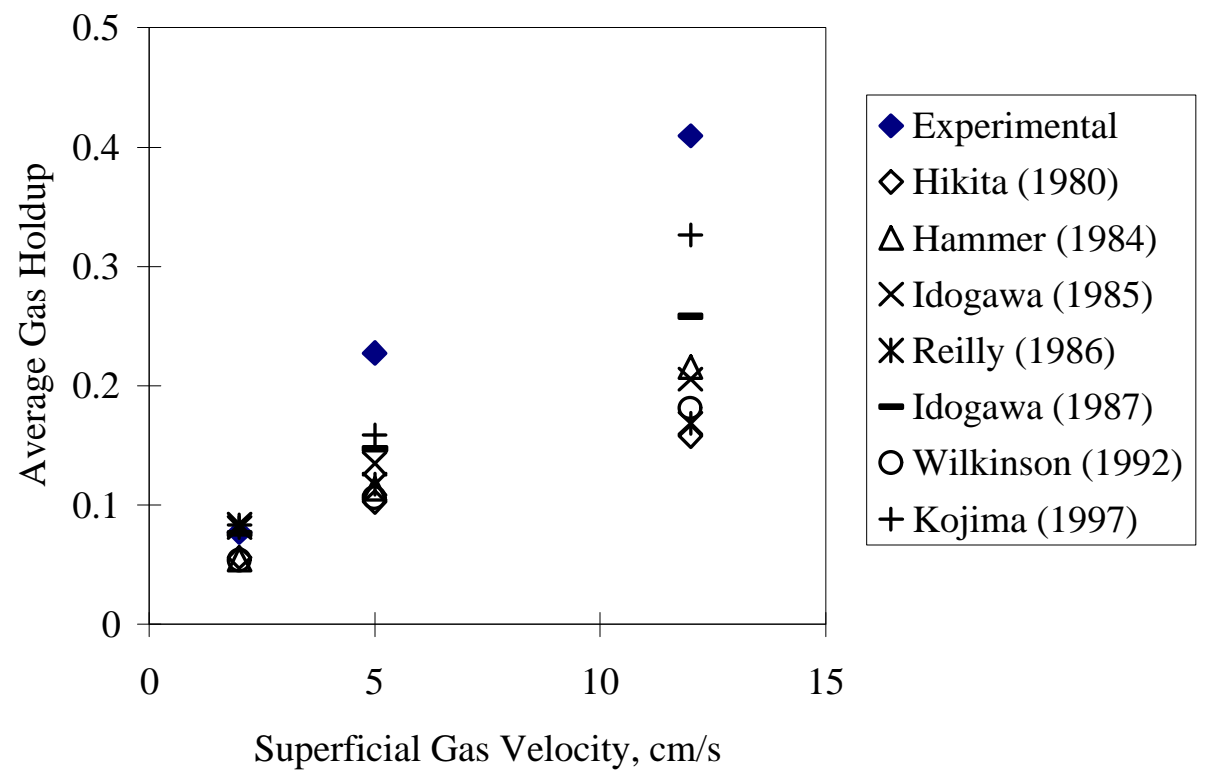

Figure 3.14: Cross-Sectional Average Gas Holdup as a Function of Superficial Gas Velocity at $\mathrm{P}=7 \mathrm{~atm}$ 
Among the correlations reported in Table 3.3 those of Idogawa et al. (1985), Idogawa et al. (1987), Wilkinson et al. (1992), and Kojima et al. (1997) were developed by considering high pressure data also. As evident from Figures 3.13 and 3.14 and Table 3.4, the gas holdup calculations based on Kojima et al. (1997)'s correlation have the least error compared to the observed cross-sectional gas holdup at elevated pressure.

\subsection{Summary}

The gas holdup and gas holdup cross-sectional distribution measurements were obtained at elevated pressure up to 7 atm using gamma-ray Computed Tomography (CT), which is available in CREL (Chemical Reaction Engineering Laboratory). Gas holdup increased as pressure increased due to a decrease in bubble sizes. Coalescence of bubbles decreased and the bubble breakup was promoted under pressurized conditions. The measured radial gas holdup distribution was flatter at a higher pressure than at atmospheric pressure. At atmospheric pressure at superficial gas velocity of $12 \mathrm{~cm} / \mathrm{s}$, the radial gas holdup distribution is parabolic, indicating churn-turbulent flow condition.

The cross-sectional average gas holdup was calculated using the collected data and compared with various correlations found in the literature. At atmospheric pressure, Akita and Yoshida's correlation was in the best agreement with data compared with other correlations, except for $\mathrm{U}_{\mathrm{g}}=5 \mathrm{~cm} / \mathrm{s}$. The calculated cross-sectional average gas holdup data is compared also with Shollenberger et al. (1995, 1997). The data is comparable except for $\mathrm{U}_{\mathrm{g}}=5 \mathrm{~cm} / \mathrm{s}$ at atmospheric pressure. This value is near the transition point, and thus the discrepancies can be large due to flow regime transitions. At higher pressure, the correlation of Kojima et al. (1997) predicted gas holdup values in reasonable agreement with the observed cross-sectional average gas holdup.

\subsection{Nomenclature}

$\begin{array}{lll}\mathrm{d}_{\mathrm{o}} & - & \text { inner diameter of single nozzle, } \mathrm{mm} \\ \mathrm{D} & - & \text { column diameter, } \mathrm{m} \\ \mathrm{g} & - & \text { gravitational acceleration, } \mathrm{m} / \mathrm{s}^{2} \\ \mathrm{P} & - & \text { system pressure, MPa } \\ \mathrm{P}_{0} & - & \text { standard atmospheric pressure } \\ \mathrm{Q} & - & \text { volumetric flowrate of gas under the condition in the bubble column, } \mathrm{m}^{3} / \mathrm{s} \\ \mathrm{U}_{\mathrm{g}} & - & \text { superficial gas velocity, } \mathrm{m} / \mathrm{s} \\ \mathrm{U}_{\mathrm{gc}} & - & \text { superficial gas velocity, } \mathrm{cm} / \mathrm{s} \\ \mathrm{U}_{1 . b .} & - & \text { slip velocity for large bubbles, } \mathrm{m} / \mathrm{s} \\ \mathrm{U}_{\mathrm{s.b}} & - & \text { slip velocity for small bubbles, } \mathrm{m} / \mathrm{s} \\ \mathrm{U}_{\text {trans }} & - & \text { velocity at regime transition, } \mathrm{m} / \mathrm{s} \\ \bar{\varepsilon} & - & \text { cross-sectional averaged gas holdup } \\ \gamma & - & \text { liquid surface tension, } \mathrm{N} / \mathrm{m} \\ \mathrm{V}_{1} & - & \text { liquid kinematic viscosity, } \mathrm{m}^{2} / \mathrm{s} \\ \rho_{\mathrm{g}} & - & \text { gas density, } \mathrm{kg} / \mathrm{m}^{3} \\ \rho_{1} & - & \text { liquid density, } \mathrm{kg} / \mathrm{m}^{3} \\ \sigma_{1} & - & \text { liquid surface tension, } \mathrm{mN} / \mathrm{m} \\ \sigma_{0} & - & \text { surface tension of water at } 20^{\circ} \mathrm{C}, \mathrm{mN} / \mathrm{m}\end{array}$




\subsection{References}

Adkins, D. R., Shollenberger, K. A., O'Hern, T. J., and Torczynski, J. R., "Pressure Effects on Bubble Column Flow Characteristics," ANS Proceedings, 1996 National Heat Transfer Conference, Technical Sessions sponsored by Thermal Hydraulics Division American Nuclear Society, 9, 318-325, Aug 3-6, 1996.

Akita, K., and Yoshida, F., 'Gas Holdup and Volumetric Mass Transfer Coefficient in Bubble Columns,' Ind. Eng. Chem. Process Des. Dev., 12, 76-80, 1973.

Deckwer, W.-D., Bubble Column Reactors, John Wiley \& Sons, New York, 1992.

Hammer, H., Schrog, H., Hektor, K., Schönau, K., Küsters, W., Soemarno, A., Sahabi, U., and Napp, W., 'New Subfunctions in Hydrodynamics, Heat \& Mass Transfer for Gas/Liquid and Gas/Liquid/Solid Chemical and Biochemical Reactors,' Front. Chem. Reac. Eng., 464, 1984.

Hikita, H., Asai, S., Tanigawa, K., Segawa, K., and Kitao, M., 'Gas Holdup in Bubble Columns,' Chem. Eng. J., 20, 59-67, 1980.

Idogawa, K., Ikeda, K., Fukuda, T., and Morooka, S., 'Effect of Gas and Liquid Properties on the Behavior of Bubbles in a Bubble Column under High Pressure,' Kag. Kog. Ronb., 11, 432, 1985.

Idogawa, K., Ikeda, K., Fukuda, T., and Morooka, S., 'Behavior of Bubbles of the AirWater System in a Column under High Pressure,' International Chem. Engng., 26, 3, 468-474, 1986.

Idogawa, K., Ikeda, K., Fukuda, T., and Morooka, S., 'Effect of Gas and Liquid Properties on the Behavior of Bubbles in a Column under High Pressure,' Int. Chem. Eng., 27, 93-99, 1987.

Jiang, P., Lin, T.-J., Luo, X., and Fan, L.-S., 'Flow Visualization of High Pressure (21 MPa) Bubble Column: Bubble Characteristics,' Trans IChemE, 73, Part A, 269-274, April 1995.

Kojima, H., Jun, S., and Hideyuki, S., 'Effect of Pressure on Volumetric Mass Transfer Coefficient and Gas Holdup in Bubble Column,' Chem. Eng. Sci., 52, 21/22, 4111-4116, 1997.

Kojima, H., Okumura, B., and Nakamura, A., 'Effect of Pressure on Gas Holdup in a Bubble Column and a Slurry Bubble Column,' J. Chem. Eng. Japan, 24, 1, 115-117, 1991.

Kölbel, H., Borchers, E., and Langemann, H., Chemie -Ing. -Techn., 33. 668. 1961. 
Kumar, S. B., Computed Tomography Measurements of Void Fraction and Modeling of the Flow in Bubble Columns, PhD Thesis, Florida Atlantic University, Boca Raton, FL, 1994.

Kumar, S. B., Moslemian, D., and Dudukovic, M. P., 'A Gamma Ray Tomographic Scanner for Imaging Void Fraction Distribution in Bubble Columns,' Flow Meas. Instr., 6, $1,61,1995$.

Kumar, S. B., Moslemian, D., and Dudukovic, M. P., 'Gas Holdup Measurements in Bubble Columns Using Computed Tomography,' AIChE J., 43, 6, 1414-1425, 1997.

Lin, T.-J., Tsuchiya, K., and Fan, L.-S., 'Bubble Flow Characteristics in Bubble Columns at Elevated Pressure and Temperature,' AIChE J., 44, 3, 545-560, March 1998.

Oyevaar, M. H., De La Rie T., Van Der Sluijs, and Westerterp, K. R., 'Interfacial Areas and Gas Holdups in Bubble Columns and Packed Bubble Columns at Elevated Pressures,' Chem. Eng. Process., 26, 1-14, 1989.

Oyevaar, M. H., and Westerterp, K. R., 'Mass Transfer Phenomena and Hydrodynamics in Agitated Gas-Liquid Reactors and Bubble Columns at Elevated Pressures: State of the Art,' Chem. Eng. Process., 25, 85-98, 1989.

Reilly, J. G., Scott, D. S., de Bruijn, T., Jain, A., and Diskorz, J., 'Correlation for Gas Holdup in Turbulent Coalescing Bubble Columns,' Can. J. Chem. Eng., 64, 705, 1986.

Shollenberger, K. A., Torczynski, J. R., Adkins, D. R., and O’Hern, T. J., "Bubble Column Measurements Using Gamma Tomography," ASME FED, Fluid Measurement and Instrumentation, 211, 25-30, 1995.

Shollenberger, K. A>, Torczynski, J. R., Adkins, D. R., O’Hern, T. J., and Jackson, N. B., "Gamma-densitometry Tomography of Gas Holdup Spatial Distribution in Industrial-Scale Bubble Columns," Chem. Eng. Sci., 52, 13, 2037-2048, 1997.

Wilkinson, P. M., Spek, A. P., and van Dierendonck, Laurant L., 'Design Parameters Estimation for Scale-Up of High-Pressure Bubble Columns,' AIChE J., 38, 4, 544-554, 1992.

Wilkinson, P. M., and van Dierendonck, Laurent L., 'Pressure and Gas Density Effects on Bubble Break-Up and Gas Hold-Up in Bubble Columns,' Chem. Engng. Sci., 45, 8, 23092315, 1990. 


\section{COMPUTER AUTOMATED RADIOACTIVE PARTICLE TRACKING IN HIGH PRESSURE BUBBLE COLUMN}

\subsection{Introduction}

A number of gas conversion processes utilize bubble columns and are operated at high pressure and temperature in order to promote the volumetric productivity. Information on the liquid hydrodynamics at high pressure is very limited. Traditionally, researchers in the field of multiphase flows have used fiber optics or electrical capacitance probes and hot wire or film anemometry for local velocity measurements. The disadvantages of such techniques are that they are intrusive and they yield only the measurements at a single point in the vicinity of the probe. The most recent non-intrusive techniques for studies of multiphase flow are Laser-Doppler Anemometry (LDA), Particle Image Velocimetry (PIV) and Computer Automated Radioactive Particle Tracking (CARPT). The LDA technique is handicapped by the multiple measurements that must be made at various points in the flow field. Both laser based techniques (LDA and PIV) suffer from severe scattering effects particularly when the gas or solids holdup are higher than $10 \%$. In such opaque multiphase flows they are not appropriate methods. At CREL, a nonintrusive technique, Computer Automated Radioactive Particle Tracking (CARPT), has been developed to measure the liquid phase velocities and turbulent parameters in bubble columns. It has been shown to be an excellent technique to study flow patterns in multiphase systems and has been used successfully to monitor liquid and solids motion in slurry bubble columns (Devanathan et al., 1990; Moslemian et al., 1992; Yang et al., 1992, 1993) in gas-solid fluidized beds (Lin et al., 1985) and in liquid-solid and gasliquid-solid fluidized beds (Limtrakul, 1996). As part of this project we have compared CARPT results with data obtained by PIV in dilute bubbly flow where PIV data can be assumed to be very accurate. Excellent agreement was established (Second Technical Annual Report). This provided the needed confidence to apply CARPT to opaque flow systems in which other techniques cannot be used.

In this report, the effect of pressure on liquid hydrodynamics in bubble columns is studied using CARPT. The velocity plots and turbulence parameters are evaluated and compared with existing data.

\subsection{High Pressure Experimental Setup}

The high pressure column fabricated at CREL was already described in Section 3.2. Here we briefly review the CARPT facility at CREL and introduce the new calibration device for high pressure operation.

\subsubsection{CARPT Facility}

Figure 4.1 shows the CARPT setup for the high-pressure column. Devanathan (1991) describes the detail of the software and hardware. In this study, a radioactive Scandium46 particle $\left(1.59 \mathrm{mg}\right.$ weight and $2.89 \mathrm{~g} / \mathrm{cm}^{3}$ in density with total strength of $491 \mu \mathrm{Ci}$ ) emitting $\gamma$ rays of constant energy at 0.89 and $1.12 \mathrm{MeV}$ was embedded into a polypropylene particle with a diameter of $2.38 \mathrm{~mm}$. In order to match the density of the liquid phase, an air void is created inside the polypropylene particle so that the composite 
density (Scandium-polypropylene-air) is equal to the density of the liquid phase being tracked. With the particle density the same as that of the liquid, the radioactive tracer particle is neutrally buoyant, and is able to mimic successfully the dynamic behavior of the recirculating liquid phase. The intersection of the gamma rays emitted by the tracer particles is continuously monitored by an array of $24 \mathrm{NaI}(\mathrm{Tl})$ scintillation detectors (5 $\mathrm{cm}$ in diameter), which are strategically located around the column. In order to determine the exact position of the tracer particle at each instant in time, calibrations are performed for each detector, providing a relationship between the distance from the detector to the particle and the intensity count received by the detector. It should be noted that for this set of runs our standard calibration method was still employed as we were still perfecting the Monte Carlo procedure. Using this calibration information and the intensities of radiation received by detectors at each sampling period $(50 \mathrm{~Hz})$, the instantaneous position of the particle is calculated by an inverse mapping algorithm. The instantaneous velocity could then be obtained by time differentiation. Ensemble averaged velocities and turbulence quantities can be calculated for all column locations upon running the experiment for many hours in order to get good statistics.

\subsubsection{Experimental Apparatus}

The high pressure bubble column system was described in Chapter 3.2 where we discussed the gas holdup measurements and results. The CARPT experiments were conducted at the conditions summarized in Table 4.1.

Table 4.1: Operating Conditions for the High Pressure System

Diameter of column, in. $(\mathrm{cm})$

Distributon

Superficial gas velocity, $\mathrm{cm} / \mathrm{s}$

Liquid (water)

Pressure, Mpa (atm)

Temperature, ${ }^{\circ} \mathrm{C}$
6.359 (16.15)

Perforated plate with 61 holes (each of $0.4 \mathrm{~mm}$ diameter), open area $0.05 \%$ (Figure 3.4)

5 and 15

Batch

$0.3(3)$

25

Before performing CARPT experiments, approximately 1500 to 2000 calibration points in the column have to be collected first. At atmospheric conditions, when the column is open, a fishing line was used to perform the calibrations. This technique is impossible to adapt to the high-pressure closed system. A new calibration device (Figure 4.2) was designed in order to accommodate the high pressure as well as to improve the accuracy of the calibration. The device can be adjusted azimuthally, radially and axially, without any gas leaks or decrease in the pressure, to obtain the calibration points anywhere in the column. In this study, the velocity plots and turbulence parameters are obtained at a 
pressure of $3 \mathrm{~atm}$, and a superficial gas velocity of $5 \mathrm{~cm} / \mathrm{s}$. Therefore, calibration was performed at the same conditions.

The calibration curves for each detector exhibit an exponential trend of particle distance to the detector versus the intensity counts as shown in Figure 4.3:

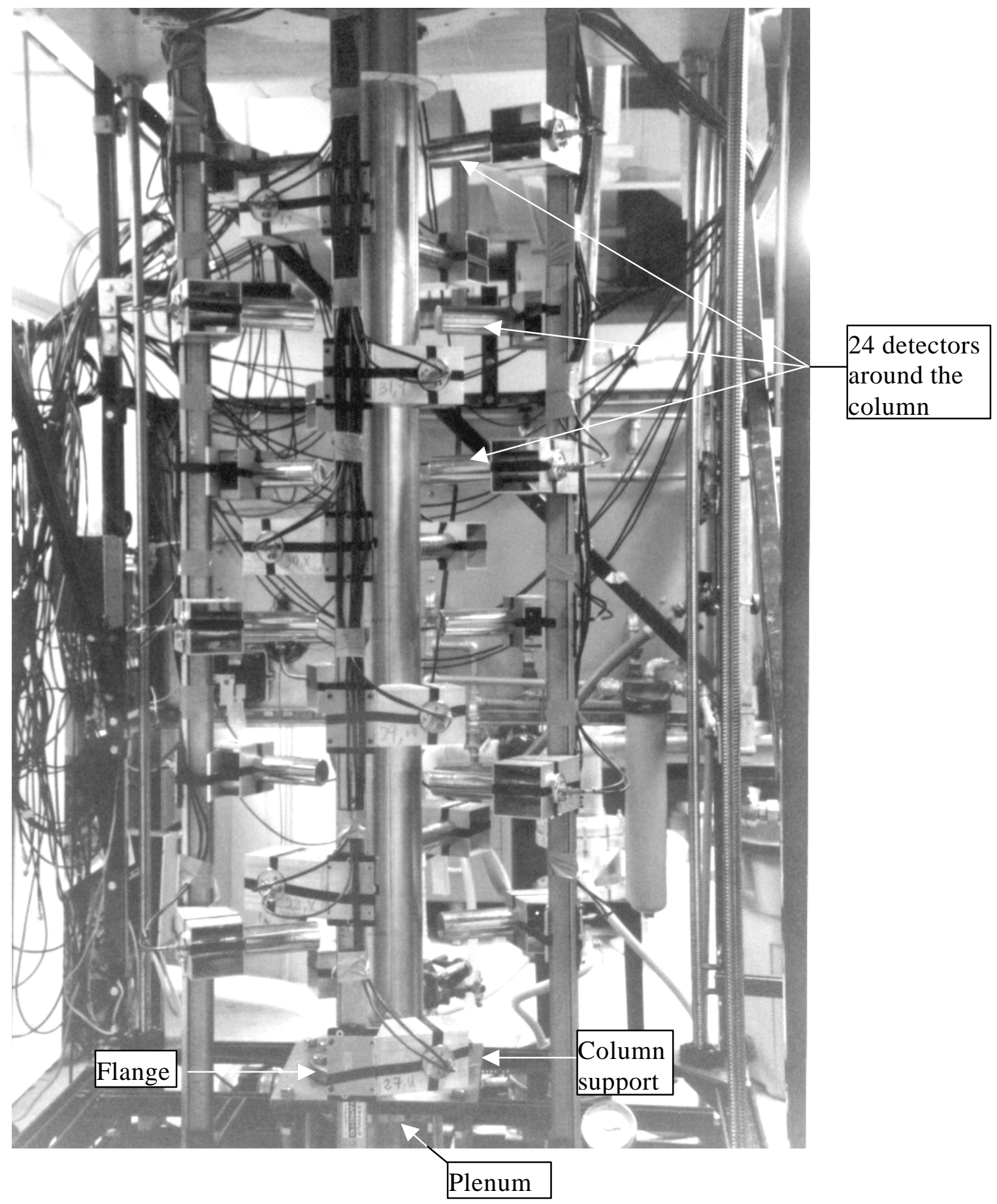

Figure 4.1. CARPT Setup for the High Pressure Bubble Column in CREL 


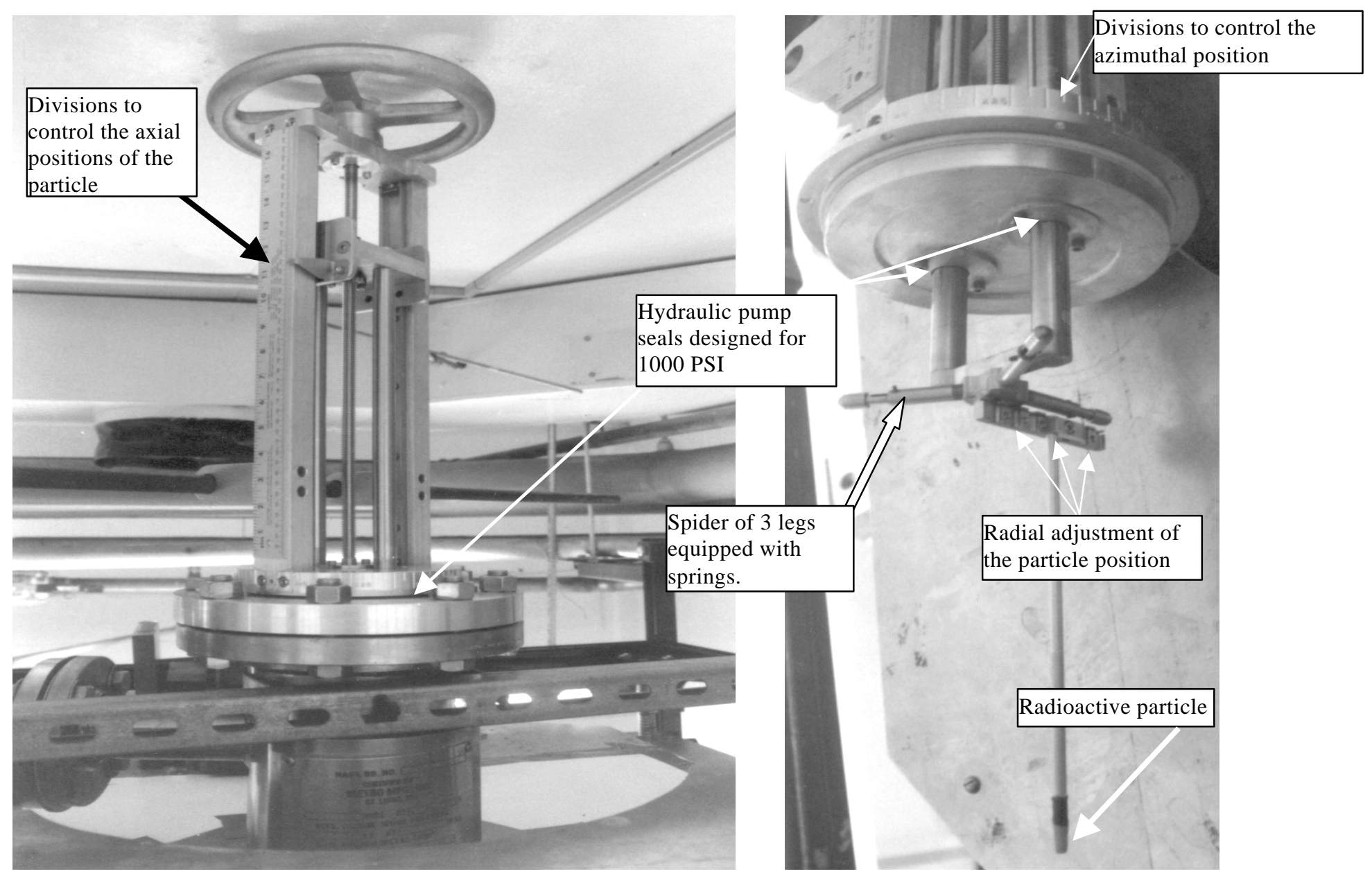

Figure 4.2: Calibration Device for High Pressure Bubble Column 


\section{Detector 9}

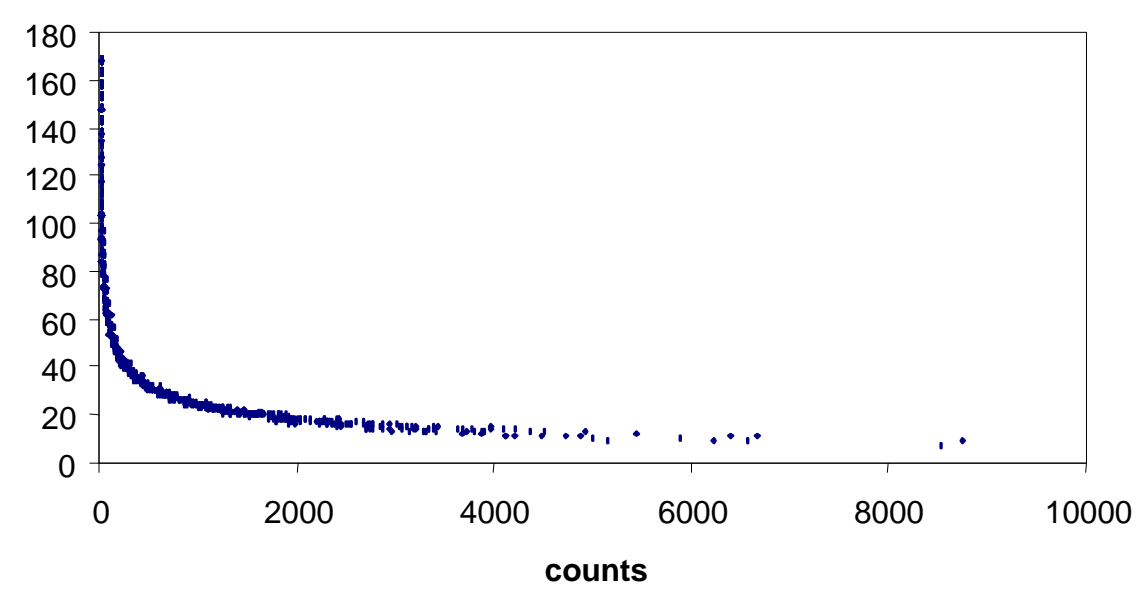

Detector 10

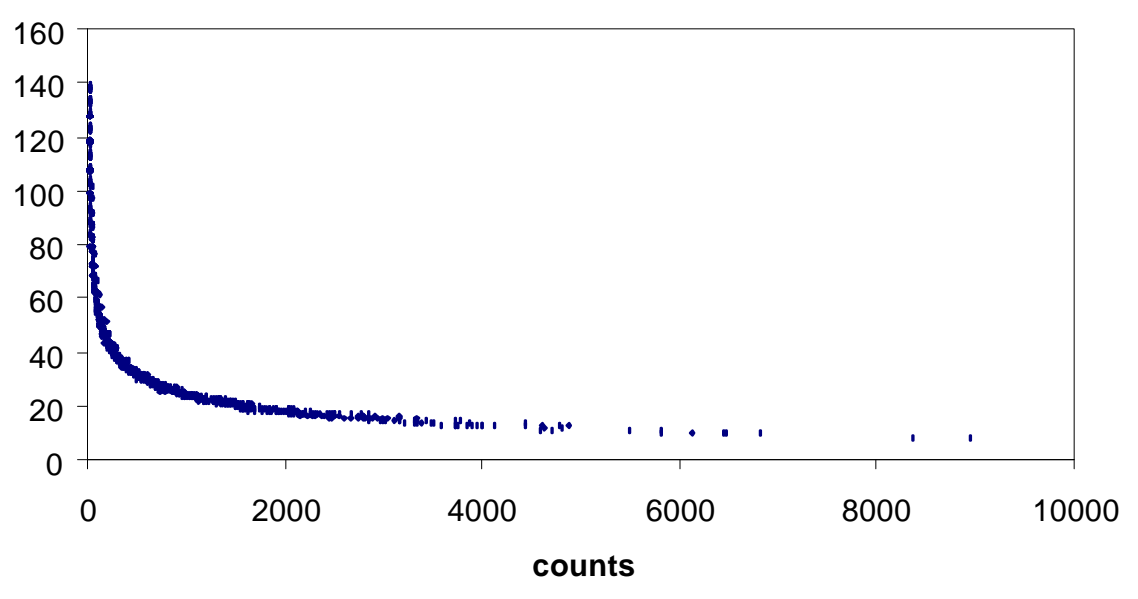

Detector 19

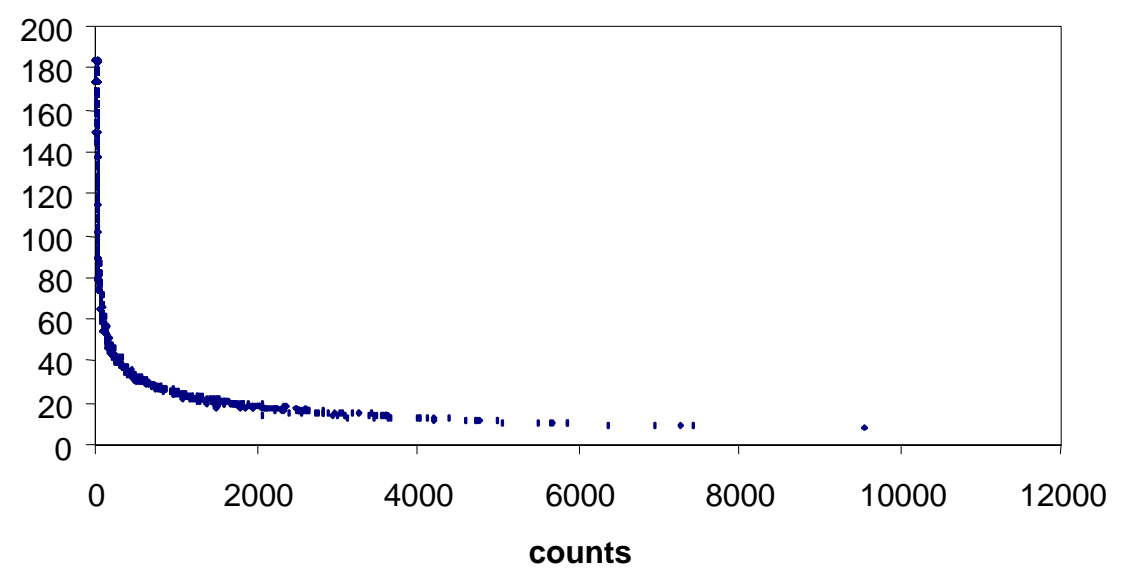

Figure 4.3: Calibration Curves of Different Detectors 
These calibration curves allow us to determine the position $(\mathrm{X}, \mathrm{Y}, \mathrm{Z})$ of the radioactive particle as a function of time, as shown in Figure 4.4:

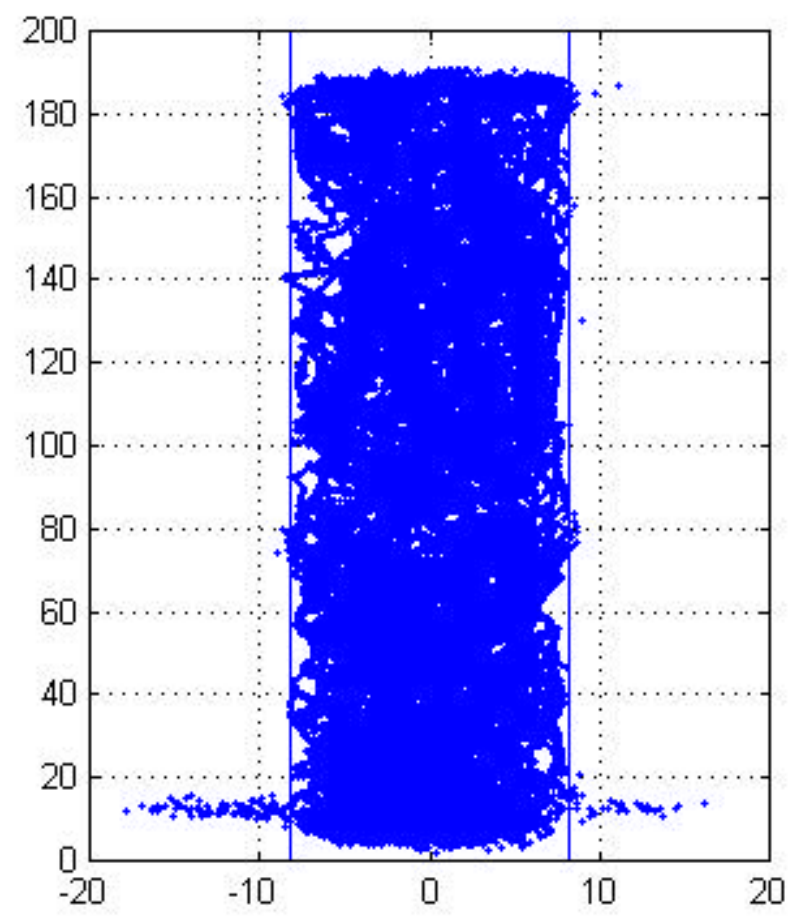

Figure 4.4: $\quad$ Tracer Particle Locations $(\mathrm{X}(\mathrm{t}), \mathrm{Z}(\mathrm{t}))$ During 20 Minutes of the Run

In the lower part of the column there are several computed positions of the tracer which are situated outside the column. This problem is due to the presence of the flange and ports in that region of the column.

During processing such data is handled appropriately as described below. Figure 4.5 shows that the accuracy near the sparger increases when the number of detectors increases in the horizontal plane situated at 6 inches above the flange.

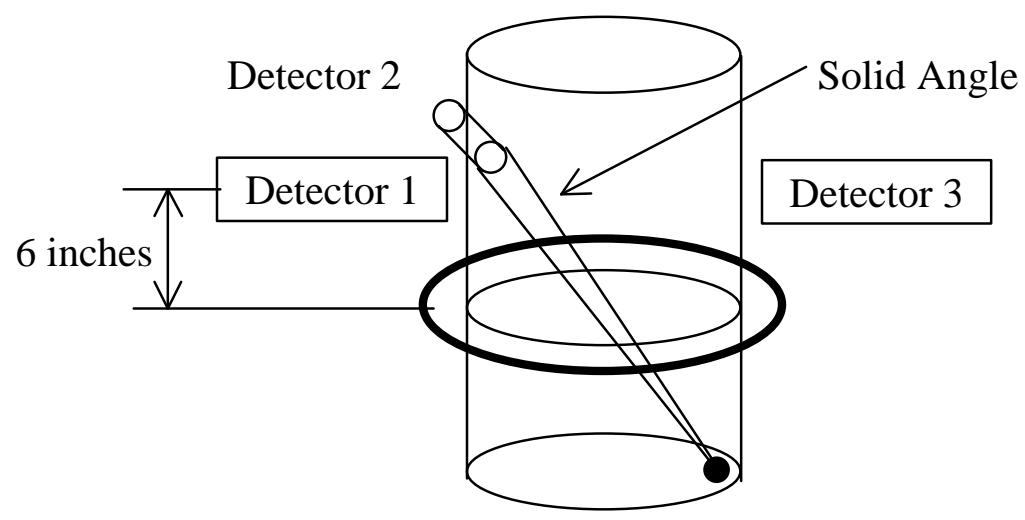

Figure 4.5: Increased Accuracy Near the Sparger with Increased Number of Detectors 
The detectors at the opposite side of the radioactive particle (detector 1 and 2) receive high counts compared to the ones close to the particle (detector 3) due to the presence of the flange. The particle position is properly computed only from the detectors situated on the left side of Figure 2.5 and the erroneous counts of the detectors such as detector 3 can be excluded from the computation of tracer position.

\subsection{Results and Discussion}

The CARPT run at $5 \mathrm{~cm} / \mathrm{s}$ superficial gas velocity at $0.3 \mathrm{MPa}$ is discussed here. The ensemble averaged axial liquid velocity profile is displayed in Figure 4.6 at two elevations of $\mathrm{z}=30 \mathrm{~cm}$ and $\mathrm{z}=90 \mathrm{~cm}$ above the distributor. Clearly the velocities are much higher in the distributor zone and the distributor effect is still noticeable at $\mathrm{z}=30$ $\mathrm{cm}$ which is the distance equal almost to two diameters. It should be noted that the gas velocity at the hole of the perforated plate is almost $100 \mathrm{~m} / \mathrm{s}$. Evidently small bubbles are injected at high speed, and are gradually slowed down by the liquid. CT scans confirm bubbly flow at these conditions and relatively flat holdup profiles. At $90 \mathrm{~cm}$ above the distributor one can safely assume that the fully developed velocity region is reached. The center line velocity is only about $5 \mathrm{~cm} / \mathrm{s}$ and reaches values of about $-7 \mathrm{~cm} / \mathrm{s}$ close to the wall, with zero averaged axial velocity at $r=5.6 \mathrm{~cm}$. In contract, Degaleesan (1997) reported (Figure 4.7a) a center line velocity of about $30 \mathrm{~cm} / \mathrm{s}$ at atmospheric pressure and gas superficial velocity of $4.8 \mathrm{~cm} / \mathrm{s}$. Her column was equipped with the same perforated plate distributor. Several phenomena contribute to this. It is well know that flow regime transition occurs earlier at lower pressure, and that velocities in the churn turbulent regime are larger. Increased pressure also decreases bubble size, leading to smaller bubble rise velocity, and increased gas density diminishes the driving force for liquid recirculation.

The fact that the axial liquid velocities obtained at atmospheric pressure at almost the same superficial gas velocity are 6 times higher than the liquid velocities obtained at 3 atm seems to indicate that the atmospheric column was in churn turbulent flow while the high pressure column was still in bubbly flow. CT scans seem to confirm this.

The behavior of liquid axial velocity profile and turbulent kinetic energy at atmospheric pressure as a function of superficial gas velocity is displayed in Figure 4.7 taken from Degaleesan (1997). Studies to determine the dependence on superficial gas velocity at elevated pressure are being planned.

The kinetic turbulent energy profile obtained from the CARPT run at $3 \mathrm{~atm}$ is shown in Figure 4.8. Specific turbulent kinetic energy decays seven fold from the high values of 350 dynes $/ \mathrm{cm}^{2}$ at $\mathrm{z}=30 \mathrm{~cm}$ to 50 dynes $/ \mathrm{cm}^{2}$ at $\mathrm{z}=90 \mathrm{~cm}$. The kinetic energy in the fully developed region is 4 to 5 times lower than found at the same superficial gas velocity at atmospheric conditions (Figure 4.7b). This also indicates that the flow at elevated pressure remains in the bubbly flow regime.

Based on these limited results we reach two important conclusions. First, CARPT can be used successfully to measure velocity and turbulence parameters in high pressure

columns. Second the flow at high pressure remains in the bubbly flow regime until 
higher superficial gas velocities are reached and this reduces the liquid recirculation rate and reduces liquid turbulence.

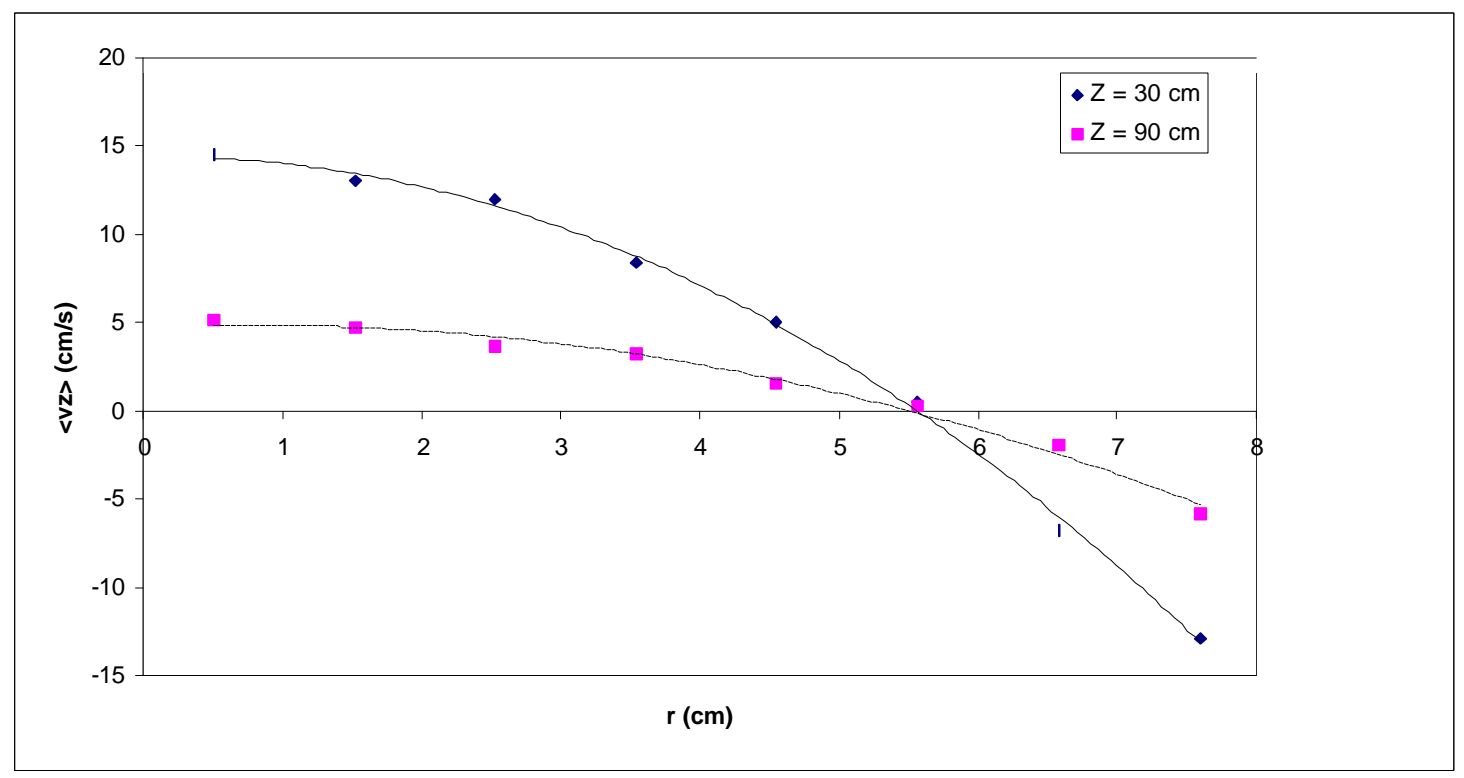

Figure 4.6: Axial Mean Velocities at Different $\mathrm{z}$ Level $(30$ and $90 \mathrm{~cm}$ ) for a Superficial Gas Velocity of $5 \mathrm{~cm} / \mathrm{s}$ at Pressure of $0.3 \mathrm{MPa}$

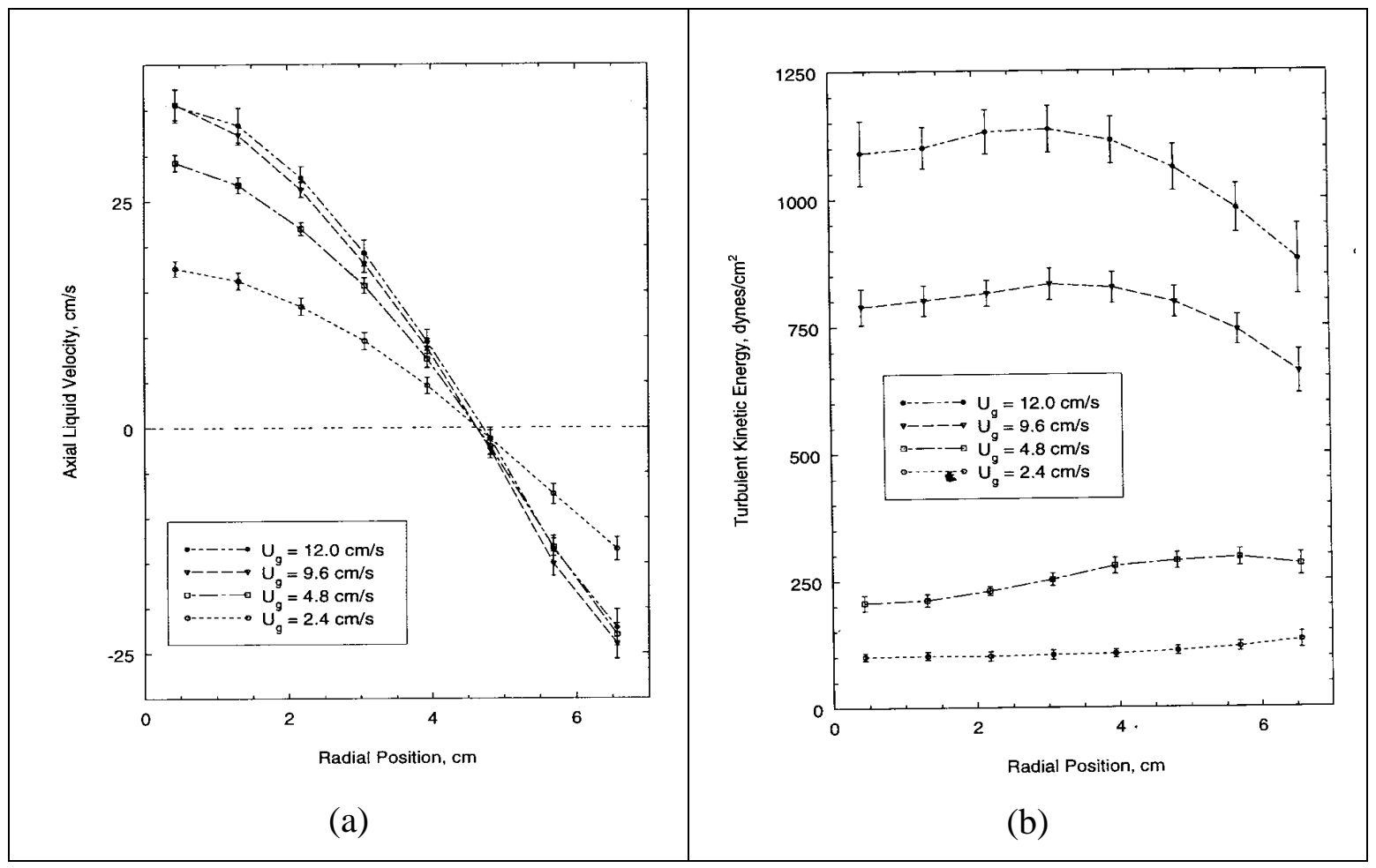

Figure 4.7: Axial Liquid Velocity Profiles (a), Turbulent Kinetic Energy (b) For an Atmospheric Column of 6 Inches Diameter 


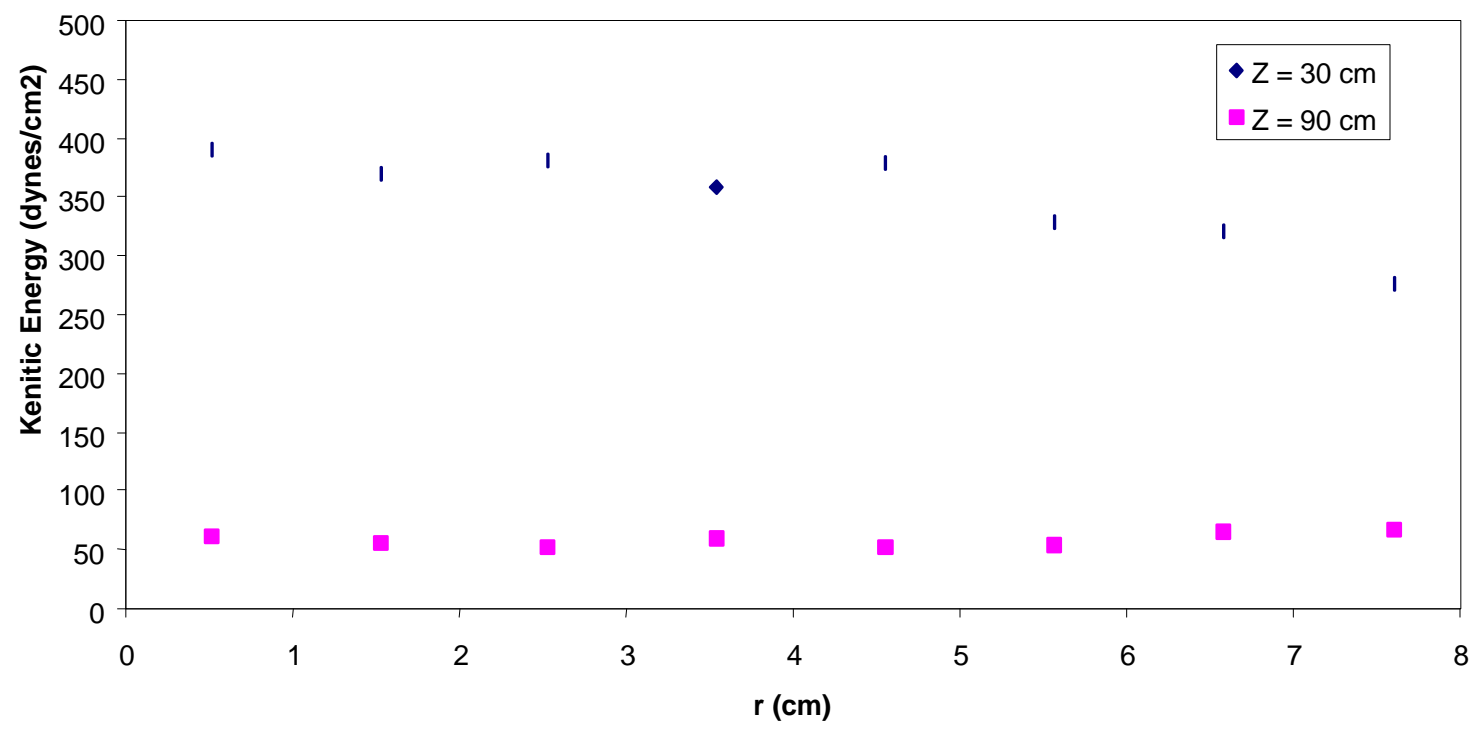

Figure 4.8: Kinetic Energy Profile. Superficial Gas Velocity: 5cm/s. Pressure:0.3 MPa

\subsection{References}

Kemoun, A., Ong, B. C., Gupta, P., Al-Dahhan, M., Dudukovic, M. P., Chang, M., "Gas Holdup in High Pressure Bubble Column by Computed Tomography," To be submitted, 1998.

Degaleesan, S., "Fluid Dynamic Measurements and Modeling of Liquid Mixing in Bubble Columns", D. Sc. Thesis, Washington University, St. Louis, Missouri USA, 1997.

Devanathan, N., Moslemian, D., Dudukovic, M. P., "Flow Mapping in Bubble Columns Using CARPT," Chem. Eng. Sci., 45, 8, 2285 - 2291, 1990.

Devanathan, N., Investigation of Liquid Hydrodyamics in Bubble Columns via Computer Automated Radioactive Particle Tracking, D. Sc. Thesis, Washington University, St. Louis, Missouri, USA, 1991.

Dudukovic, M. P., Degaleesan, S., Gupta, P., Kumar, S. B., "Fluid Dynamics in ChurnTurbulent Bubble Columns - Measurements and Modeling," Proceedings of the ASME Fluids Engineering Division, Summer Meeting, Vancouver, Canada, 244, 3517, 1997.

Limtrakul, S., Hydrodynamics of Liquid Fluidized Beds and Gas-Liquid Fluidized Beds, D. Sc. Thesis, Washington University, St. Louis, Missouri, USA, 1996. 
Lin, J., S., Chen, M., Chao, B. T., "A Novel Radioactive Particle Tracking Facility for Measurement of Solids Motion in Gas Fluidized Beds, AIChE J., 31, 2, 465 - 473, 1985.

Moslemian, D., Devanathan, N., Dudukovic, M. P., "Radioactive Particle Tracking Technique for Investigation of Phase Recirculation and Turbulence in Multiphase Systems," Rev. Sci. Instrum., 63, 10, 4361 - 4372, 1992.

Yang, Y. B., Devanathan, N., Dudukovic, M. P., "Liquid Backmixing in Bubble Columns," Chem. Eng. Sci., 47, (9,11), 2859 - 2864, 1992.

Yang, Y. B., Devanathan, N., Dudukovic, M. P., "Liquid Backmixing in Bubble Columns via Computer Automated Radioactive Particle Tracking (CARPT)," Exp. Fluids, 16, 1-9, 1993. 


\section{MONTE CARLO SIMULATIONS OF NaI(TL) SCINTILLATION \begin{tabular}{llllll} 
DETECTORS & FOR & MULTI-PHASE & FLOW & MAPPING & AND \\
\hline
\end{tabular} VISUALIZATION USING CARPT}

A Monte Carlo based simulation strategy has been developed for determining the total and photopeak efficiencies of cylindrical $\mathrm{NaI}(\mathrm{Tl})$ scintillation detectors for an arbitrarily located point $\gamma$-ray source in the three-dimensional space. Improved computational efficiency, in evaluating the intrinsic photopeak efficiency of the detectors, has been achieved by using Gaussian quadratures for carrying out multi-dimensional integration, instead of the frequently used uniform sampling in conventional Monte Carlo methods. It is observed that the photopeak efficiency is not a constant, and varies with the position of the $\gamma$-ray point source. A generalized reduced gradient optimization scheme has been applied to optimize for the detector gains and dead-times, which are needed for the construction of a detailed 3-D calibration map to dynamically locate the position of the radioactive particle. An efficient computational scheme has also been developed to compute the particle position from the dynamic count data. This scheme uses a chi-square minimization in conjunction with 3-D interpolation to provide improved accuracy in locating the particle position.

\subsection{Introduction}

The Computer Automated Radioactive Particle Tracking (CARPT) has been proven to be an excellent tool for studying the flow pattern/mixing mechanisms in multiphase reactors (Devanathan, 1991; Devanathan et al., 1990, Dudukovic et al., 1997). Improvements and changes in the CARPT facility are made from time to time to make it suitable to study various reactor systems. One issue that has always been a bottleneck in the use of CARPT, is the need for an in-situ calibration procedure. Traditional implementation of the technique requires a tedious and time-consuming calibration at each operating condition in the reactor geometry under investigation. During calibration for a given operating condition, the current procedure requires the construction of a distance-count map for each detector, by placing a radioactive particle, the flow follower, in a few hundred to a thousand or more known locations over the entire reactor volume. This is currently achieved by mounting the radioactive particle on fishing lines, fixed between two grids at the two ends of the reactor, and manually moving the particle to various locations in the column. All the calibration data are taken at the operating conditions of interest in order for the distance-count maps to properly reflect the hold-up variations. Once the entire calibration map is available for each detector, the dynamic position of this tracer particle can be computed from the instantaneous counts data acquired by the detectors. Timedifferentiation of the instantaneous position data provides the instantaneous velocity of the particle. The application of the ergodic hypothesis to the ensemble-averaged data results in the evaluation of the time-averaged velocity and turbulence-parameter fields over the entire reactor volume.

The Monte Carlo simulation of detector photopeak efficiencies offers an alternative to this tedious in-situ calibration procedure. It is based on an approach where the radioactive- 
count received by a detector is modeled, as opposed to a heuristically based current procedure. When a radioactive particle is used as a phase follower in multiphase flows, the intervening medium between the point source and the detector consists of the reactor media and the reactor wall (as well as insulation if present). Thus, appropriate geometrical arguments have to be used to compute the probability of non-interaction of the $\gamma$-ray photon with the media in between the radioactive source and the detector. Additionally, the technique has the advantage of determining the dynamic location of the radioactive particle from the counts registered by all the detectors, using previously constructed (obtained by using the Monte Carlo simulations) 3-D position-counts map for each detector. This improves the accuracy of locating the particle position from count data received by each detector, since 3-D mapping is being used instead of 1-D mapping from in-situ calibration.

\subsection{Research Objectives}

The literature on the application of Monte Carlo technique for simulating the photo-peak efficiency of a cylindrical $\mathrm{NaI}(\mathrm{Tl})$ detector in response to a single point source has been well studied. It is the aim of this work to extend this firm theoretical basis by applying it to more complex geometries and media configurations, as present in a reactor with multiphase flow. Specifically, the objectives are:

1) Develop Monte Carlo programs to calculate the intrinsic efficiency of a cylindrical $\mathrm{NaI}(\mathrm{Tl})$ detector due to a point source located at any position inside the reactor volume.

2) Validate these programs by comparing the simulation results from this work with the available Monte Carlo simulation as well as experimental data for a point source located on the axis of a cylindrical detector.

3) Verify the simulation results by comparing the simulated counts to those measured at a number of known locations inside a reactor. Optimize detector dead-time constants, detector gains and the linear attenuation coefficient of the reactor media (function of the local media density) to best match the experimental and theoretical data.

4) Develop programs to generate 3-D position-count calibration maps for each detector using the optimized variables obtained above for each detector.

5) Generate source codes to dynamically determine the particle position by inverse mapping the counts from each detector against the position-count map obtained from Monte Carlo simulations above.

6) Verify the accuracy of the inverse-mapping programs by comparing the predicted particle position with its actual position by placing the radioactive particle into several known locations.

7) Integrate all the programs developed above to process data from actual CARPT experiments. 


\subsection{Mathematical Description}

The basic framework for implementation of this technique has been provided by Beam et al. (1978). They discuss the application of Monte Carlo simulation for the calculation of efficiencies of right-circular cylindrical $\mathrm{NaI}$ detectors for arbitrarily located $\gamma$-ray emitting radioactive point sources. They successfully show that Monte Carlo calculations can provide $\mathrm{NaI}$ detector efficiencies at any specified energy, without resorting to tedious experimental measurements. In this work, the framework established by Beam et al. has been modified to include the presence of the reactor walls, and the two or three phase mixture in the reactor. A similar implementation of the Monte Carlo approach has been demonstrated to be successful by Larachi et al. (1994).

The development of Beam et al. (1978) does not include the effects due to the cladding material encasing the scintillation crystal or the photo-multiplier mounting. At this moment, these effects are not included in this work either, but some researchers have shown that these effects may be significant, especially when simulating the entire energy spectrum (Nardi, 1970; Steyn et al., 1973; Saito and Moriuchi, 1981). On the other hand, if one is interested in simulating only the photo-peak portion of the energy spectrum, the results appear to be insensitive to the inclusion or non-inclusion of the effects of the cladding material in the simulations. Therefore, the non-inclusion of these effects could be justified for the moment. However, efforts are underway to modify the existing codes to include the presence of the cladding material in order to quantitatively analyze the effect of the cladding material. Also, Beam's analysis considers only Compton and photoelectric interactions, while the production of secondary electrons is neglected. This implies that the photon energies should be less than $1 \mathrm{MeV}$. This is true of the radioactive particles being used for CARPT $\left(\mathrm{Sc}^{46}\right)$ and therefore, presents no limitation. In what follows, a brief description of the theoretical basis for this work is discussed.

The Monte Carlo treatment consists of following and categorizing a large number of photon histories from emission at the source to absorption within the detector. Random number and probability theory, combined with known transport distributions are used to locate the photon collision site, as well as trajectory, energy and direction through each history. As developed by Beam et al. (1978), three variance reduction steps are employed during each history:

- Each $\gamma$-ray is forced to strike the detector.

- Each $\gamma$-ray is forced to interact within the bounds of the detector; i.e., photons are not allowed to escape from the detector.

- Each interaction is forced to be a Compton Scattering event.

A photon history is terminated when either the weight of a scattering interaction (ratio of scattering to total cross-section) or the energy of the photon falls below a specified minimum (e.g. $10^{-10}$ or $0.01 \mathrm{MeV}$ ). Any possibility of bias due to these variance reduction techniques is eliminated by calculating the appropriate weights for each of the above forced events using well-defined physical and geometrical principles. 


\subsubsection{Determination of Solid Angle}

The determination of the solid angle at an arbitrary point by a cylindrical detector can be accomplished by a Monte Carlo calculation. Figure 5.1 shows the position of the point source relative to the detector, which is a known basic input.

The two cases that have to be considered are:

- A point source located in such a position so that the $\gamma$-ray photons can enter from the top as well as the side (Figure 5.1).

- A point source located in such a position so that the $\gamma$-ray photons can enter only from the top (Figure 5.2).

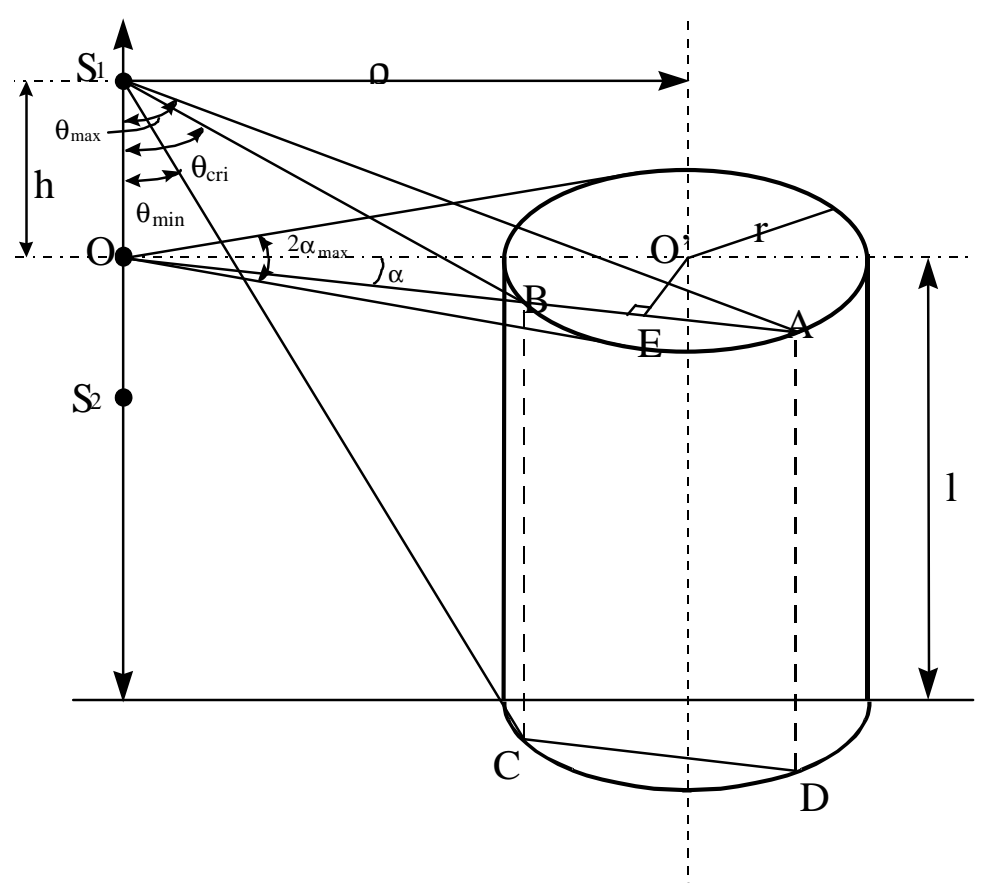

Figure 5.1: Notation Used in the Selection of Angles for Monte Carlo Calculations

From Figure 5.1, we see that if $\rho$ is the distance from the center of the detector to a line parallel to the detector axis, but which contains the point source, and $r$ is the detector radius, then we can define the angle $\alpha_{\max }$ as

$\alpha_{\max }=\sin ^{-1}(r / \rho)$

The actual angle $\alpha$ is derived from

$\mathrm{n}=\int_{-\alpha_{\max }}^{\alpha} \frac{\mathrm{d} \alpha}{2 \pi} / \int_{-\alpha_{\max }}^{\alpha_{\max }} \frac{\mathrm{d} \alpha}{2 \pi}$ 
where, $n$ is a random number selected from a uniform distribution between 0 and 1 . Consequently,

$\alpha=\alpha_{\max }(2 n-1) \quad-\alpha_{\max } \leq \alpha \leq \alpha_{\max }$

The weighting factor associated with this selection of $\alpha, w(\alpha)$, is given by

$\mathrm{w}(\alpha)=\frac{\int_{-\alpha_{\max }}^{\alpha_{\max }} \frac{\mathrm{d} \alpha}{2 \pi}}{\int_{0}^{2 \pi} \frac{\mathrm{d} \alpha}{2 \pi}} \quad \mathrm{w}(\alpha)=\frac{\alpha_{\max }}{\pi}$

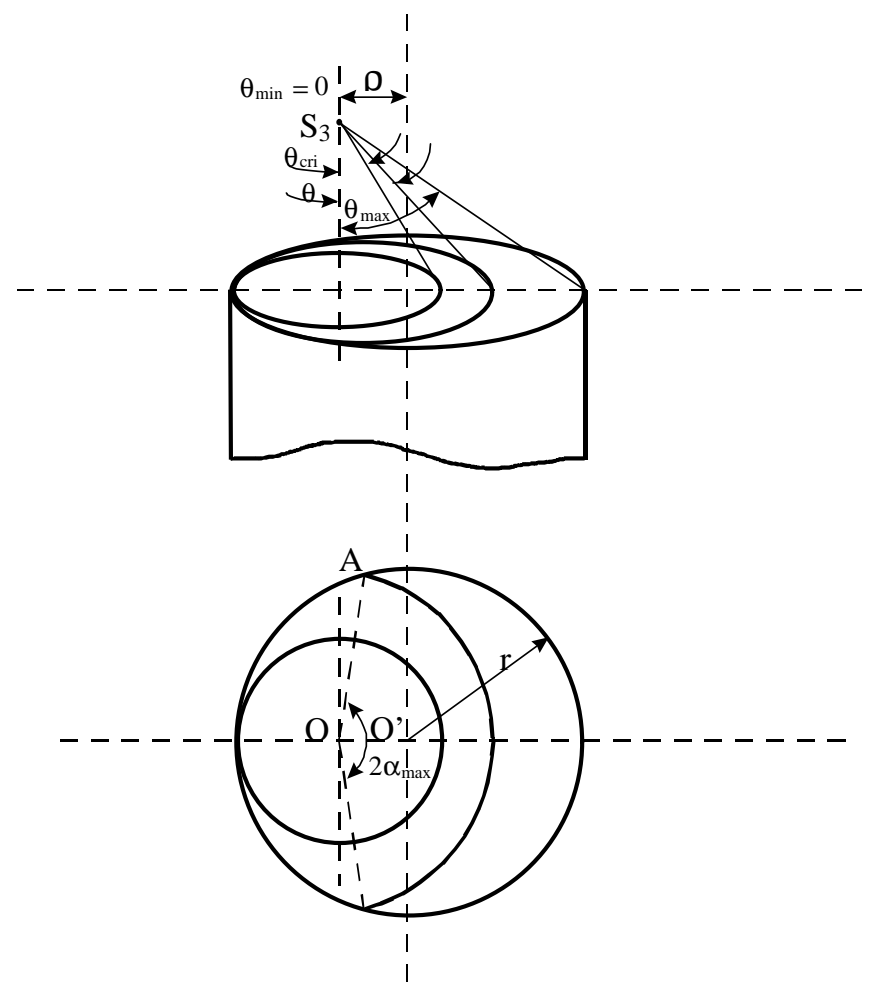

Figure 5.2: $\quad$ Notation for the Case of a Point Source Located Directly Above the Circular Face of the Detector

Once $\alpha$ is randomly selected, the points A, B, C, and D in Figure 5.1 define the plane through which the photon must enter the detector for point sources located at $S_{1}$ and $S_{2}$ (Beam et al., 1978). Next, one has to define the angles $\theta_{\max }, \theta_{c r i}$ and $\theta_{\min }$ to determine the position along the plane through which the photon enters. From Figure 5.1, the line segments $O A$ and $O B$ can be defined as

$\mathrm{OA}=\rho \cos \alpha+\left(r^{2}-\rho^{2} \sin ^{2} \alpha\right)^{1 / 2}$

$\mathrm{OB}=\rho \cos \alpha-\left(r^{2}-\rho^{2} \sin ^{2} \alpha\right)^{1 / 2}$

Several cases need to be considered. 
Case I When the source is located above the top plane of the detector, for example at $S_{1}$ (i.e., when $h \geq 0$ )

$\theta_{\text {max }}=\tan ^{-1}(O A / h), \theta_{c r i}=\tan ^{-1}(O B / h), \theta_{\text {min }}=\tan ^{-1}(O B /(h+l))$

Case II When the source is located on the top plane of the detector, for example at $O$ (i.e., when $h=0$ )

$\theta_{\max }=\pi / 2, \theta_{\text {cri }}=\pi / 2, \theta_{\min }=\tan ^{-1}(\mathrm{OB} / 1)$

Case III When the source is located below the top plane of the detector, for example at $S_{2}$ (i.e., when $h<0$ )

$\theta_{\max }=\pi / 2+\tan ^{-1}(|h| / O B), \theta_{c r i}=\pi / 2+\tan ^{-1}(|h| / O B), \theta_{\text {min }}=\tan ^{-1}(O B /(l-|h|))$

The particular angle $\theta$, which defines the angle along which the photon enters the detector, is chosen using another rectangularly distributed random number $n^{\prime}$.

$$
\mathrm{n}^{\prime}=\frac{\int_{\theta_{\min }}^{\theta} \frac{\sin \theta}{2} \mathrm{~d} \theta}{\int_{\theta_{\min }}^{\theta_{\max }} \frac{\sin \theta}{2} \mathrm{~d} \theta} \quad \text { or } \quad \theta=\cos ^{-1}\left\{\cos \left(\theta_{\min }\right)-\mathrm{n}^{\prime}\left[\cos \left(\theta_{\min }\right)-\cos \left(\theta_{\max }\right)\right]\right\}
$$

$\theta$ has to be tested to see if the photon enters the top or the side of the detector by comparing it to the angle $\theta_{c r i}$ defined above. The appropriate weighting factor, $w(\theta)$, for this selection of $\theta$ is given by

$$
\mathrm{w}(\theta)=\frac{\int_{\theta_{\min }}^{\theta_{\max }} \frac{\sin \theta}{2} \mathrm{~d} \theta}{\int_{0}^{\pi} \frac{\sin \theta}{2} \mathrm{~d} \theta} \quad \text { or } \quad \mathrm{w}(\theta)=\frac{\cos \left(\theta_{\min }\right)-\cos \left(\theta_{\max }\right)}{2}
$$

Case IV When the source is located on top of the detector face, for example at $S_{3}$ as in Figure 2

$\theta_{\max }=\tan ^{-1}[(r+\rho) / h], \theta_{c r i}=\tan ^{-1}[(r-\rho) / h], \theta_{\text {min }}=0$

Here, the critical angle, $\theta_{c r i}$ defines an angle above which the variation of $\alpha$ is limited to $2 \alpha_{\max }$ and below which angle $\alpha$ may vary over $2 \pi$. In the first case, the associated weighting factor, $w(\alpha)$ is the same as defined before, but for the second case, $w(\alpha)$ is 
simply equal to 1 , as $\alpha_{\max }$ is equal to $\pi$. Therefore, in this case, $\theta$ is calculated first and $\alpha$ is determined knowing the value of $\theta$. Stated mathematically, it translates to

$$
\begin{array}{lll}
\text { For } \theta>\theta_{\text {cri }}, & \alpha_{\max }=\cos ^{-1} \frac{\rho^{2}+\mathrm{h}^{2} \tan ^{2} \theta-\mathrm{r}^{2}}{2 \mathrm{~h} \rho \tan \theta} \\
\text { For } \theta \leq \theta_{\text {cri }}, & \alpha_{\text {max }}=\pi
\end{array}
$$

Finally, the total weighting factor for any selection of $\alpha$ and $\theta$ in Figures 5.1 and 5.2 is given by

$W_{i}=w(\theta) w(\alpha)$

Where, $W_{i}$ represents the solid angle subtended for this particular selection of $\alpha$ and $\theta$. The estimate of the solid angle, $\Omega$, is given by the mean value

$$
\Omega=\frac{4 \pi}{\mathrm{N}} \sum_{\mathrm{i}=1}^{\mathrm{N}} \mathrm{W}_{\mathrm{i}}
$$

where, $N$ is the total number of histories.

In our implementation of the Monte Carlo method, instead of choosing the photon histories along uniformly distributed (rectangular) random directions as in Beam et al. (1978), the angles $\alpha$ and $\theta$ are chosen from the Gaussian distributed pseudo-random directions. This reduces the computational demands by around an order of magnitude. If $i$ corresponds to the $\alpha$-coordinate and $j$ to the $\theta$-coordinate, then

$$
\mathrm{x}_{\mathrm{g}}(\mathrm{i})=2 \mathrm{n}-1, \quad \mathrm{x}_{\mathrm{g}}(\mathrm{j})=2 \mathrm{n}^{\prime}-1, \quad-1 \leq \mathrm{x}_{\mathrm{g}}(\mathrm{i}) \leq 1,-1 \leq \mathrm{x}_{\mathrm{g}}(\mathrm{j}) \leq 1
$$

where, the two random numbers, $n$ and n', are replaced with Gaussian points, $\mathrm{x}_{\mathrm{g}}(\mathrm{i})$ and $\mathrm{x}_{\mathrm{g}}(\mathrm{j})$ respectively. It has been shown in this work that the results for the photo-peak efficiency are within $1 \%$ accuracy with 30 Gaussian points when compared to standard base results computed using 200 Gaussian points.

\subsubsection{Photon Interaction with Reactor Media and Detector Crystal}

Once the solid angle computation has been accomplished, the next thing to consider is the calculation of the probability of certain type of interaction. We must assess:

a) The probability that $\gamma$-rays emitted within $\Omega$ would not interact with the reactor media (gas-liquid, gas-liquid-solid mixture) and the reactor wall, $\mathrm{f}_{\mathrm{a}}$

$f_{a}(\alpha, \theta)=\exp \left[\sum_{i=1}^{n} \mu_{i} d_{i}(\alpha, \theta)\right]$

where, 
$\mu_{\mathrm{i}} \equiv$ total linear attenuation coefficient of the material $\mathrm{i}$ in the $\gamma$-ray path.

$\mathrm{d}_{\mathrm{i}} \equiv$ distance traveled by the gamma-ray in the direction $(\alpha, \theta)$ through reactor media/wall.

$\alpha \equiv$ angle with the line normal to detector axis.

$\theta \equiv$ angle with the detector axis.

b) The probability of interaction (Compton +Photo-Peak) of gamma-rays, emitted within the solid angle, with the detector crystal, $\mathrm{f}_{\mathrm{d}}$

$f_{a}(\alpha, \theta)=1-\exp \left[-\mu_{d} d(\alpha, \theta)\right]$

where,

$\mu_{\mathrm{d}} \equiv$ total linear attenuation coefficient of the detector crystal.

$\mathrm{d} \equiv$ distance traveled through the detector by an undisturbed gamma-ray in the direction $(\alpha, \theta)$

c) The probability of photo-peak interaction of gamma-rays, emitted within the solid angle, with the detector crystal, $\mathrm{f}_{\mathrm{p}}$

$$
\begin{aligned}
& \mathrm{f}_{\mathrm{p}}=\mathrm{w}_{1} \frac{\tau_{1}}{\mu_{1}}+\mathrm{w}_{v} \frac{\tau_{v}}{\mu_{v}} \prod_{\mathrm{j}=2}^{v} \mathrm{w}_{\mathrm{j}-1} \frac{\sigma_{\mathrm{j}-1}}{\mu_{\mathrm{j}-1}} \\
& \mathrm{w}_{\mathrm{j}}=1-\exp \left[-\mu_{\mathrm{j}} \mathrm{d}_{\text {eff }}\right]
\end{aligned}
$$

where,

$\mathrm{w}_{1} \equiv$ equal to $\mathrm{f}_{\mathrm{d}}$.

$\mathrm{d}_{\text {eff }} \equiv$ effective distance traveled by gamma-ray inside the detector.

$\sigma_{\mathrm{j}} \equiv$ attenuation coefficient due to Compton interaction.

$\tau_{\mathrm{j}} \equiv$ attenuation coefficient due to Photo-peak interaction.

$\mu_{j}=\sigma_{j}+\tau_{j} \equiv$ total attenuation coefficient.

$\sigma_{j} . \tau_{j} \cdot u_{j} \equiv$ these are all functions of gamma-rav energv. and have to be recomputed after each Compton interaction which changes the gamma-ray (photon)

Having defined $f_{a}, f_{d}$ and $f_{p}$, the total and photo-peak efficiencies can be calculated by evaluating the following 3_D integrals

$$
\begin{aligned}
& \mathrm{T}_{\varepsilon}=\iint_{\Omega} \frac{\overrightarrow{\mathrm{r}} \cdot \overrightarrow{\mathrm{n}}}{\mathrm{r}^{3}} \mathrm{f}_{\mathrm{a}}(\alpha, \theta) \mathrm{f}_{\mathrm{d}}(\alpha, \theta) \mathrm{ds} \\
& \mathrm{P}_{\varepsilon}=\iint_{\Omega} \frac{\overrightarrow{\mathrm{r}} \cdot \overrightarrow{\mathrm{n}}}{\mathrm{r}^{3}} \mathrm{f}_{\mathrm{a}}(\alpha, \theta) \mathrm{f}_{\mathrm{p}}(\alpha, \theta) \mathrm{ds}
\end{aligned}
$$

where, 
$\vec{r} \equiv$ vector from the point source to a variable point $\mathrm{p}$ on the exposed detector surface.

$\mathrm{n} \equiv$ external unit vector locally normal to the surface at point $\mathrm{p}$.

ds $\equiv$ differential area element around point $\mathrm{p}$.

The angles $\alpha$ and $\theta$, as described in Figures 5.1 and 5.2 need to be related to the direction cosines of the $\gamma$-ray path from the tracer position to the entry point on the detector surface. This is necessary for determining the distance a $\gamma$-ray travels inside the reactor and through the reactor wall. Since the detector axis for all the detectors are perpendicular to the reactor axis, axes rotations and transformations are implemented to make these calculations tractable.

The origin of the initial coordinate system is the center of the reactor bottom plane, z-axis is along the length of the column, and the $\mathrm{x}-\mathrm{y}$ plane forms the horizontal cross-section of the column. For any particle position $\left(\mathrm{x}_{\mathrm{p}}, \mathrm{y}_{\mathrm{p}}, \mathrm{z}_{\mathrm{p}}\right.$ inside the reactor) and detector location $\left(\mathrm{x}_{\mathrm{c}}, \mathrm{y}_{\mathrm{c}}, \mathrm{z}_{\mathrm{c}}\right.$ outside the reactor), the following axis rotations and transformations are performed:

1) Rotation on $x-y$ plane by an angle $\omega$ ' to make the detector axis parallel to the new $x^{\prime}-$ axis:

$$
\begin{array}{ll}
\omega^{\prime}=\tan ^{-1}\left(\frac{y_{c}}{x_{c}}\right) & x_{c}>0 \\
\omega^{\prime}=\frac{\pi}{2} & x_{c}=0 \\
\omega^{\prime}=\pi+\tan ^{-1}\left(\frac{y_{c}}{x_{c}}\right) & x_{c}<0
\end{array}
$$

The particle and detector positions in the new coordinate system are $x_{p}^{\prime}, y_{p}^{\prime}$ and $x_{c}^{\prime}, y_{c}^{\prime}$, respectively (z position is not changed):

$$
\begin{aligned}
& \mathrm{x}_{\mathrm{p}}^{\prime}=\mathrm{x}_{\mathrm{p}} \cos \omega^{\prime}+\mathrm{y}_{\mathrm{p}} \sin \omega^{\prime} \\
& \mathrm{y}_{\mathrm{p}}^{\prime}=-\mathrm{x}_{\mathrm{p}} \sin \omega^{\prime}+\mathrm{y}_{\mathrm{p}} \cos \omega^{\prime} \\
& x_{c}^{\prime}=x_{c} \cos \omega^{\prime}+y_{c} \sin \omega^{\prime} \\
& y_{c}^{\prime}=0
\end{aligned}
$$

The distance $\mathrm{h}$ between the center of the detector face to the tracer location and the radius $\rho$, indicating the distance of the tracer from the detector axis, are given by:

$$
\mathrm{h}=\left|\mathrm{x}_{\mathrm{c}}^{\prime}-\mathrm{x}_{\mathrm{p}}^{\prime}\right|, \quad \rho=\sqrt{\left(\mathrm{y}_{\mathrm{c}}^{\prime}-\mathrm{y}_{\mathrm{p}}^{\prime}\right)^{2}+\left(\mathrm{z}_{\mathrm{c}}^{\prime}-\mathrm{z}_{\mathrm{p}}^{\prime}\right)^{2}}
$$


The equation of the circle for the reactor perimeter in the horizontal cross section remains the same, i.e.,

$$
x^{2}+y^{2}=R_{i}^{2}\left(\text { or } R_{0}^{2}\right)
$$

where, $R_{i}$ and $R_{o}$ are the reactor inner and outer radii, respectively.

2) Rotation in the $y^{\prime}-z^{\prime}$ plane by an angle $\omega^{\prime \prime}$ to for the projection of the 3-D line $\left(\mathrm{x}_{\mathrm{p}}^{\prime}, \mathrm{y}_{\mathrm{p}}^{\prime}, \mathrm{z}_{\mathrm{p}}^{\prime}\right)$ to $\left(\mathrm{x}_{\mathrm{c}}^{\prime}, \mathrm{y}_{\mathrm{c}}^{\prime}, \mathrm{z}_{\mathrm{c}}^{\prime}\right)$ on the $\mathrm{y}^{\prime}-\mathrm{z}^{\prime}$ plane parallel to the new $\mathrm{z}^{\prime \prime}$ axis:

$$
\begin{array}{ll}
\omega^{\prime \prime}=\tan ^{-1}\left(\frac{\mathrm{y}_{\mathrm{c}}^{\prime}-\mathrm{y}_{\mathrm{p}}^{\prime}}{\mathrm{z}_{\mathrm{c}}^{\prime}-\mathrm{z}_{\mathrm{p}}^{\prime}}\right) & \text { if } \mathrm{z}_{\mathrm{c}}^{\prime} \neq \mathrm{z}_{\mathrm{p}}^{\prime} \\
\omega^{\prime \prime}=\frac{\pi}{2} & \text { if } \quad \mathrm{z}_{\mathrm{c}}^{\prime}=\mathrm{z}_{\mathrm{p}}^{\prime}
\end{array}
$$

The tracer particle (subscript p) and the detector (subscript c) positions in the new coordinate system are:

$$
\begin{aligned}
& \mathrm{x}_{\mathrm{p}}^{\prime \prime}=\mathrm{x}_{\mathrm{p}}^{\prime} \\
& \mathrm{y}_{\mathrm{p}}^{\prime \prime}=-\mathrm{z}_{\mathrm{p}}^{\prime} \sin \omega^{\prime \prime}+\mathrm{y}_{\mathrm{p}}^{\prime} \cos \omega^{\prime \prime} \\
& \mathrm{z}_{\mathrm{p}}^{\prime \prime}=\mathrm{z}_{\mathrm{p}}^{\prime} \cos \omega^{\prime \prime}+\mathrm{y}_{\mathrm{p}}^{\prime} \sin \omega^{\prime \prime} \\
& x_{c}^{\prime \prime}=x_{c}^{\prime} \\
& y_{c}^{\prime \prime}=-z_{c}^{\prime} \sin \omega^{\prime \prime}+y_{c}^{\prime} \cos \omega^{\prime \prime} \\
& z_{c}^{\prime \prime}=z_{c}^{\prime} \cos \omega^{\prime \prime}+y_{c}^{\prime} \sin \omega^{\prime \prime}
\end{aligned}
$$

The equation of the circle for the reactor perimeter in the horizontal cross section in the new coordinate system becomes:

$$
x^{2}+\left(-z \sin \omega^{\prime \prime}+y \cos \omega^{\prime \prime}\right)^{2}=R_{i}^{2}\left(\text { or } R_{0}^{2}\right)
$$

The direction cosines $\left(\cos \alpha^{\prime \prime}, \cos \beta^{\prime \prime}, \cos \gamma^{\prime \prime}\right)$ of the $\gamma$-ray path from the tracer location to the entry point on the detector can now be related to the angles $\alpha$ and $\theta$ (from the detector point of view) by:

$\cos \alpha^{\prime \prime}=\cos \theta_{1}, \quad \cos \beta^{\prime \prime}=\sin \theta_{1} \sin \alpha, \quad \cos \gamma^{\prime \prime}=\sin \theta_{1} \cos \alpha$

where, $\theta_{1}=\theta$ if $\mathrm{z}_{\mathrm{p}}^{\prime \prime} \leq \mathrm{z}_{\mathrm{c}}^{\prime \prime}, \quad$ otherwise, $\quad \theta_{1}=\pi-\theta$. 
After knowing the direction cosines of the path (particle to $\gamma$-ray entry point), the path equations can be written as:

$$
\begin{aligned}
& x=x_{p}^{\prime \prime}+t \cos \alpha^{\prime \prime} \\
& y=y_{p}^{\prime \prime}+t \cos \beta^{\prime \prime} \\
& z=z_{p}^{\prime \prime}+t \cos \gamma^{\prime \prime}
\end{aligned}
$$

where, $\mathrm{t}$ is the parameter defining the line in a 3-D space.

These linear equations can be solved along with the reactor circle equation to obtain the intersection point of the $\gamma$-ray path with the reactor inner diameter, ID, and the reactor outer diameter, OD. Once these parametric equations are substituted into the circle equation, one gets a quadratic equation in $t$ (parameter defining a line in 3-D) which has an analytical solution. Once $t$ is known, the equations above, defining the 3-D line, are used to compute the intersection points with the reactor inner and outer walls. There are two intersection points for each circle equation. The one closer to the detector is the true solution, whereas the other is discarded. The distance traveled by a $\gamma$-ray inside the reactor media, $d_{r}$, and through the reactor wall, $d_{w}$, can then be determined by the particle position and intersection points, i.e.

$$
\begin{aligned}
& \mathrm{d}_{\mathrm{r}}=\sqrt{\left(\mathrm{x}_{\mathrm{p}}^{\prime \prime}-\mathrm{x}_{\mathrm{id}}^{\prime \prime}\right)^{2}+\left(\mathrm{y}_{\mathrm{p}}^{\prime \prime}-\mathrm{y}_{\mathrm{id}}^{\prime \prime}\right)^{2}+\left(\mathrm{z}_{\mathrm{p}}^{\prime \prime}-\mathrm{z}_{\mathrm{id}}^{\prime \prime}\right)^{2}} \\
& \mathrm{~d}_{\mathrm{w}}=\sqrt{\left(\mathrm{x}_{\mathrm{p}}^{\prime \prime}-\mathrm{x}_{\mathrm{od}}^{\prime \prime}\right)^{2}+\left(\mathrm{y}_{\mathrm{p}}^{\prime \prime}-\mathrm{y}_{\mathrm{od}}^{\prime \prime}\right)^{2}+\left(\mathrm{z}_{\mathrm{p}}^{\prime \prime}-\mathrm{z}_{\mathrm{od}}^{\prime \prime}\right)^{2}}-\mathrm{d}_{\mathrm{r}}
\end{aligned}
$$

where, $\left(x_{i d}, y_{i d}, z_{i d}\right)$ is the intersection point with the reactor inner wall, ID, and $\left(x_{\text {od }}, y_{\text {od }}\right.$, $\left.\mathrm{Z}_{\mathrm{od}}\right)$ is the intersection with the reactor outer wall, OD. 

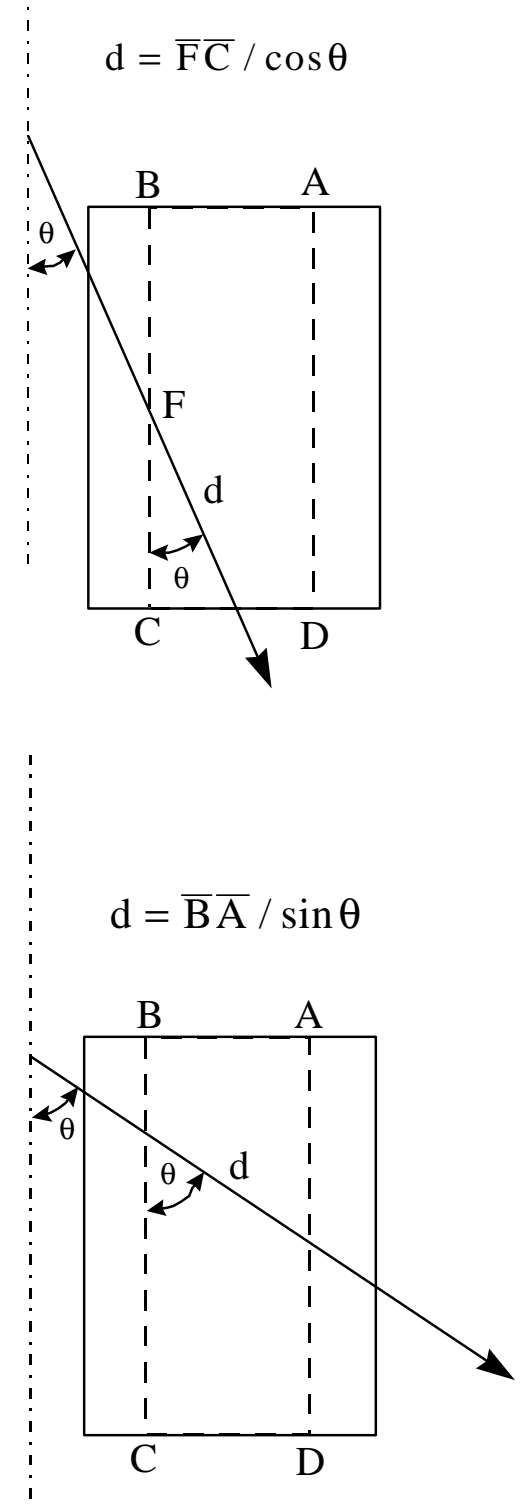
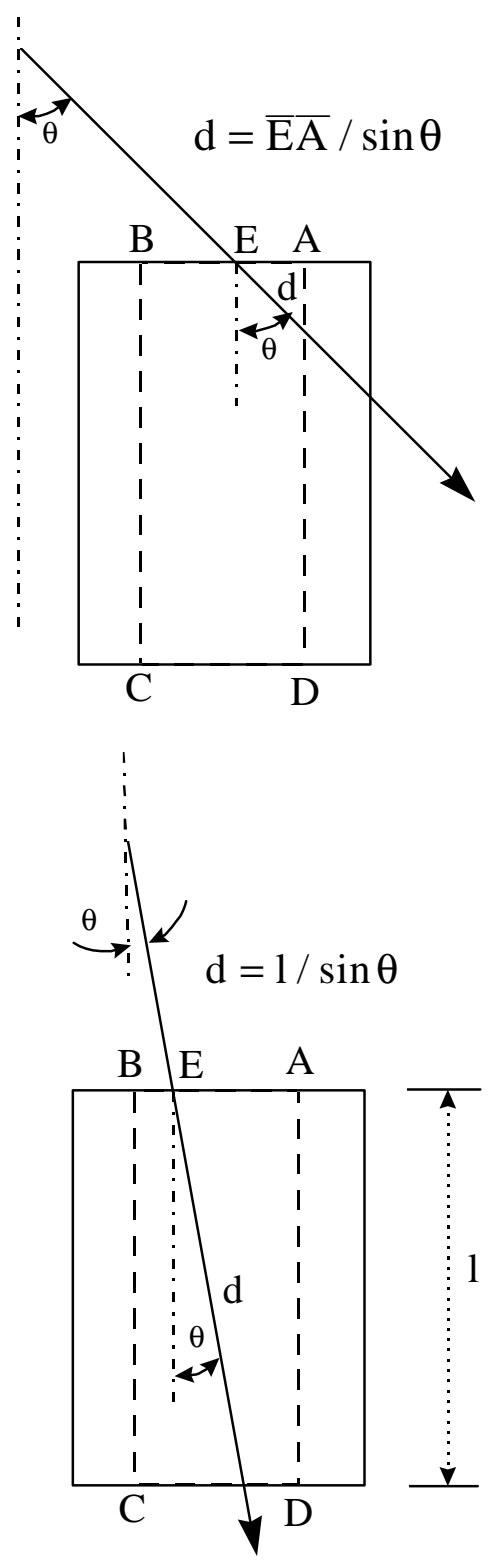

Figure 5.3: The Four Possible Cases of Travel of Photons Through the Detector

Having calculated the distances a photon travels through the reactor media and through the reactor wall, one has to determine next the location on the detector surface where the photon is going to enter the crystal. Subsequently, one needs to determine where the undisturbed $\gamma$-ray is going to exit the detector. Depending on h, $\alpha, \rho$ and $\theta$, there could be four possible combinations of where a photon enters and where it exits the detector crystal (Figure 5.3). Through firm mathematical arguments (not presented here), one determines the effective distance, $\mathrm{d}$, which a photon travels through the detector undisturbed, as well as the initial direction cosines and the first interaction site of the photon with the crystal (which is the already known position where the photon enters the crystal). 
With the initial direction cosines and the site of the first interaction known, the locations of the subsequent interaction sites are obtained from

$\mathrm{X}_{\mathrm{N}+1}=\mathrm{l}^{\prime} \cos \alpha+\mathrm{X}_{\mathrm{N}}, \quad \mathrm{Y}_{\mathrm{N}+1}=\mathrm{l}^{\prime} \cos \beta+\mathrm{Y}_{\mathrm{N}}, \quad \mathrm{Z}_{\mathrm{N}+1}=\mathrm{l}^{\prime} \cos \gamma+\mathrm{Z}_{\mathrm{N}}$

In the above equations, $\cos \alpha, \cos \beta$ and $\cos \gamma$ are the direction $\operatorname{cosines}$ after the $\mathrm{N}^{\text {th }}$ interaction, $l^{\prime}$ is the photon path length between interaction sites, which is determined from another rectangularly distributed random number between 0 and $1, n "$

$$
\mathrm{n}^{\prime \prime}=\frac{\int_{0}^{1^{\prime}} \mathrm{e}^{-\mu \mathrm{x}} \mathrm{dx}}{\int_{0}^{\mathrm{d}} \mathrm{e}^{-\mu \mathrm{x}} \mathrm{dx}} \quad \text { or } \quad \mathrm{l}^{\prime}=\frac{-\ln \left\{1-\mathrm{n}^{\prime \prime}\left(1-\mathrm{e}^{-\mu \mathrm{d}}\right)\right\}}{\mu}
$$

The energy of a photon is reduced in accordance with the Klein-Nishina differential scattering cross-section (not discussed here). The scattered angle $\theta$ is given by the Compton scattering law

$\cos \theta=1+0.511 / \mathrm{E}_{0}-0.511 / \mathrm{E}$

where, $E_{0}$ is the initial photon energy before scattering, and $E$ is the photon energy afterwards. The azimuthal angle of the scattered path relative to the incident path is found at random from 0 to $2 \pi$ by using another rectangularly distributed random number n"'

$\phi=2 \pi n " '$

The new direction cosines after scattering are

$\cos \alpha=\cos \alpha \cos \theta+(\cos \alpha \cos \gamma \sin \theta \cos \phi-\cos \beta \sin \theta \sin \phi) /\left(1-\cos ^{2} \gamma\right)^{1 / 2}$

$\cos \beta^{\prime}=\cos \beta \cos \theta+(\cos \beta \cos \gamma \sin \theta \cos \phi+\cos \alpha \sin \theta \sin \phi) /\left(1-\cos ^{2} \gamma\right)^{1 / 2}$

$\cos \gamma=\cos \gamma \cos \theta-\sin \theta \cos \phi\left(1-\cos ^{2} \gamma\right)^{1 / 2}$

However, when $\left(1-\cos ^{2} \gamma\right)$ approaches zero, the following degenerate form is used:

$\cos \alpha=\sin \theta \cos \phi$

$\cos \beta^{\prime}=\sin \theta \sin \phi$

$\cos \gamma=\cos \gamma \cos \phi$

With the new direction cosines, and the coordinates of the new interaction site, the new distance a photon can travel from the interaction site to the wall of the detector along the scattering path is required. This is done in the same way as while calculating the distance $\mathrm{d}$, which a photon travels through the detector undisturbed.

This process of computing new direction cosines, and new interaction sites is continued until either the photon energy, $\mathrm{E}$ drops to $0.01 \mathrm{MeV}$, or the ratio of scattering to total cross-section drops to $10^{-10}$. This process is repeated for all the Gaussian points in both 
the directions, and the integrals evaluated for the total and photo-peak efficiencies. Table 5.1 lists the differences in our formulation from that of Beam et al. (1978).

Table 5.1: Comparison of Beam's Formulation to the Formulation Used in This Work

\begin{tabular}{|c|c|}
\hline Method of Beam et al (1978) & Method used in this work \\
\hline$\Omega=\frac{4 \pi}{\mathrm{N}} \sum_{\mathrm{i}=1}^{\mathrm{N}} \mathrm{w}\left(\alpha_{\mathrm{i}}, \theta_{\mathrm{i}}\right)$ & $\Omega=\pi \sum_{\mathrm{i}=1}^{\mathrm{n}} \sum_{\mathrm{j}=1}^{\mathrm{n}} \mathrm{w}_{\mathrm{g}}(\mathrm{i}) \mathrm{w}_{\mathrm{g}}(\mathrm{j}) \mathrm{w}\left(\alpha_{\mathrm{i}}, \theta_{\mathrm{j}}\right)$ \\
\hline $\mathrm{T}_{\varepsilon}=\frac{1}{N} \sum_{i=1}^{\mathrm{N}} \mathrm{w}\left(\alpha_{\mathrm{i}}, \theta_{\mathrm{i}}\right) \mathrm{f}_{\mathrm{a}}\left(\alpha_{\mathrm{i}}, \theta_{\mathrm{i}}\right) \mathrm{f}_{\mathrm{d}}\left(\alpha_{\mathrm{i}}, \theta_{\mathrm{i}}\right)$ & $\begin{array}{l}\mathrm{T}_{\varepsilon}=\frac{1}{4} \sum_{\mathrm{i}=1}^{\mathrm{n}} \sum_{\mathrm{j}=1}^{\mathrm{n}} \mathrm{w}_{\mathrm{g}}(\mathrm{i}) \mathrm{w}_{\mathrm{g}}(\mathrm{i}) \mathrm{w}\left(\alpha_{\mathrm{i}}, \theta_{\mathrm{j}}\right) \mathrm{f}_{\mathrm{a}}\left(\alpha_{\mathrm{i}}, \theta_{\mathrm{j}}\right) \mathrm{f}_{\mathrm{d}}\left(\alpha_{\mathrm{i}}, \theta_{\mathrm{j}}\right) \\
\mathrm{P}_{\varepsilon}=\frac{1}{4} \sum^{\mathrm{n}} \sum_{\mathrm{i}}^{\mathrm{n}} \mathrm{w}_{\mathrm{g}}(\mathrm{i}) \mathrm{wg}_{\mathrm{g}}(\mathrm{i}) \mathrm{w}\left(\alpha_{\mathrm{i}}, \theta_{\mathrm{j}}\right) \mathrm{f}_{\mathrm{a}}\left(\alpha_{\mathrm{i}}, \theta_{\mathrm{j}}\right) \mathrm{f}_{\mathrm{p}}\left(\alpha_{\mathrm{i}}, \theta_{\mathrm{j}}\right)\end{array}$ \\
\hline $\mathrm{P}_{\varepsilon}=\frac{1}{N} \sum_{i=1}^{N} w\left(\alpha_{i}, \theta_{i}\right) f_{a}\left(\alpha_{i}, \theta_{i}\right) f_{p}\left(\alpha_{i}, \theta_{i}\right)$ & $\begin{aligned} w_{g}(i) \equiv & \text { weight corresponding to the Gaussian } \\
& \text { Xg }(i), \text { which corresponds. }\end{aligned}$ \\
\hline $\begin{aligned} \alpha_{\mathrm{i}}, \theta_{\mathrm{i}} \equiv & \text { determined by the tracer } \\
& \text { and the point where the gamma- } \\
& \text { ray enters the }\end{aligned}$ & $\begin{aligned} \mathrm{w}_{\mathrm{g}}(\mathrm{j}) \equiv & \text { weight corresponding to the Gaussian } \\
& \mathrm{Xg}(\mathrm{j}) \text {, which corresponds } \\
\mathrm{n}= & \text { number of Gaussian noints in each }\end{aligned}$ \\
\hline $\begin{aligned} \mathrm{N} \equiv & \text { number of randomly } \\
& \text { gamma-ray histories, } \\
& 10000 \text { for accurate }\end{aligned}$ & $\begin{array}{l}\sim 30 \text { are enough to give accurate value } \\
\text { the multi-dimensional }\end{array}$ \\
\hline
\end{tabular}

\subsubsection{Computation of Simulated Counts}

Once the detector photo-peak efficiency has been simulated, the radioactive counts registered by each detector are evaluated. The number of $\gamma$-ray peaks received by $\mathrm{NaI}$ detector obeys the nonparalyzable model, and the detector count is mathematically expressed as:

$\mathrm{C}=\frac{\mathrm{TV}^{*} \mathrm{GRP}_{\varepsilon}}{1+\tau v^{*} \mathrm{GRP}_{\varepsilon}}$

$\mathrm{T} \equiv$ sampling time.

$v^{*} \equiv$ number of $\gamma$-rays emitted per disintegration ( 2 for $\mathrm{Sc}^{46)}$.

$\mathrm{G} \equiv$ detector gain factor.

$\mathrm{R} \equiv$ source strength (activity), disintegrations/second.

$\mathrm{P}_{\varepsilon} \equiv$ photo-peak efficiency or full-energy peak efficiency.

$\tau \equiv$ dead time of the detector. 


\subsubsection{Optimization}

In calculating the simulated counts, $\mathrm{C}_{\mathrm{ij}}$, the gain factor, $\mathrm{G}$ and the dead-time, $\tau$, are known only approximately. Also, the effective attenuation coefficient through the reactor medium depends on the local gas hold-up profile (representative of distribution of the reactor media density, which is unknown). Thus, one has to resort to an optimization technique to get the optimal values of $\mathrm{G}, \tau$ and the three parameters in the universal gas hold-up profile

$$
\begin{aligned}
& \varepsilon_{g}(\zeta)=\widetilde{\varepsilon}_{g}\left(\frac{m+2}{m}\right)\left(1-c \zeta^{m}\right) \\
& \bar{\varepsilon}_{g}=\widetilde{\varepsilon}_{g}\left(\frac{m+2-2 c}{m}\right)
\end{aligned}
$$

Although the holdup profile in two-phase bubble columns is known to deviate from the form proposed above (generally, true only in the well developed section of the column), the variations with the reactor axis are not known to be significant except near the distributor and disengagement zones. As the variations in these zones are not well quantified and modeled, for this work at the moment, the variation of the profile with the column axis has been neglected.

For each detector, the objective function, to be minimized, is defined as:

$$
O B J_{i}=\sum_{j=1}^{N_{c a l i}} W_{i j}\left(\frac{\left(C_{i j}-M_{i j}\right)}{\left(C_{i j}+M_{i j}\right) / 2}\right)^{2}
$$

$\mathrm{M}_{\mathrm{ij}} \equiv$ measured counts.

$\mathrm{C}_{\mathrm{ij}} \equiv$ simulated counts.

$\mathrm{W}_{\mathrm{ij}}=\frac{\sqrt{\mathrm{W}_{\mathrm{ii}}}}{\sum_{\mathrm{i}=1}^{\mathrm{N}_{\text {cali }}} \sqrt{\mathrm{W}_{\mathrm{ii}}}} \equiv$ weighting factor for detector $\mathrm{i}$ and calibration point $\mathrm{j}$.

The following approximation in the evaluation of the photo-peak efficiency has been used for computational efficiency. When the results are compared to the case when no approximation is used, the errors rarely exceed $1 \%$.

$$
P_{\varepsilon}=\iint_{\Omega} \frac{\vec{r} \cdot \vec{n}}{r^{3}} f_{a}(\alpha, \theta) f_{p}(\alpha, \theta) d s \approx \iint_{\Omega} \frac{\vec{r} \cdot \vec{n}}{r^{3}} f_{p}(\alpha, \theta) d s \iint_{\Omega} \frac{\vec{r} \cdot \vec{n}}{r^{3}} f_{a}(\alpha, \theta) d s
$$

The discretized form of the above integral becomes

$$
\mathrm{P}_{\varepsilon} \approx \frac{1}{4} \sum_{i=1}^{\mathrm{n}} \sum_{j=1}^{\mathrm{n}} \mathrm{w}_{\mathrm{g}}(\mathrm{i}) \mathrm{w}_{\mathrm{g}}(\mathrm{j}) \mathrm{w}\left(\alpha_{\mathrm{i}}, \theta_{\mathrm{j}}\right) \times \frac{1}{4} \sum_{\mathrm{i}=1}^{\mathrm{n}} \sum_{\mathrm{j}=1}^{\mathrm{n}} \mathrm{w}_{\mathrm{g}}(\mathrm{i}) \mathrm{w}_{\mathrm{g}}(\mathrm{j}) \mathrm{w}\left(\alpha_{\mathrm{i}}, \theta_{\mathrm{j}}\right) \mathrm{f}_{\mathrm{p}}\left(\alpha_{\mathrm{i}}, \theta_{\mathrm{j}}\right) \mathrm{f}_{\mathrm{a}}\left(\alpha_{\mathrm{i}}, \theta_{\mathrm{j}}\right)
$$

The above optimization for our case is being implemented through a generalized reduced gradient method using the code GRG2. Once the optimization routines successfully 
converge to provide the optimal values of the optimized variables, a 3-D distance-count map is generated for each detector. The map could be created for as fine a resolution as desired, limited only by the finite size of the neutrally-buoyant radioactive flow follower, the statistical nature of the radiation, and constraints of computer memory and storage costs.

With the calibration map already available, an actual experiment is carried out in which a neutrally-buoyant tracer is let free in the flow, and the counts emitted by it are registered by each detector at finite time intervals ( $20 \mathrm{~ms}$ for a usual CARPT experiment). Programs have been developed to compute the chi-squared values of the measured counts against those from the calibration map. The location from the calibration map, which provides the minimum chi-squared value, is taken as a coarse estimation of the particle position at that instant of time. To get the exact particle position, a 3-D interpolation and minimization using Powell's routine are implemented on the chi-squared values of the 26 closest neighbors of the above point (the mathematical details are not being provided here). Thus, the particle location is accurately estimated by this procedure at every instant of time.

\subsection{Theoretical Validation}

The computer codes required for optimization, calculation of photo-peak efficiencies, and particle position reconstruction from dynamic counts data have been validated against theoretically simulated data. The details of the validation results are presented elsewhere (Gupta, 1997). The two important results from the simulations are

- The peak to total efficiency ratio is not a constant and is dependent on the location of the source with respect to the detector.

- Thirty point Gaussian quadrature in each direction during a surface integration, are sufficient to accurately simulate the photo-peak of each detector.

\subsection{Experimental Validation}

The ultimate test of the reliability of any numerical technique based on physical models is achieved when one validates the programs with real experimental data. As the Monte Carlo procedure systematically models the photo-peak fraction (or the counts associated with the photo-peak), it is necessary that while acquiring the counts during an experimental run, the thresholds and sampling windows are correctly set so as to sample just the photo-peak counts. This is best achieved by measuring the emitted energy spectrum from a point source using a Multi Channel Analyzer (MCA) so that the start and end of the photo-peak could easily be identified. Since $\mathrm{Sc}^{46}$ does not emit photons having energy above $1.2 \mathrm{MeV}$, the possibility of pair production is rare and therefore, one does not need to worry too much about the end of the photo-peak. The only thing one has to control though, is the threshold, or the start of the photo-peak, and adjust the hardware settings correctly so as to properly sample the requisite counts. Experimental data for verification of the Monte Carlo approach presented here was acquired in a cylindrical Plexiglas column with an i.d. of 7.47" and o.d. of 8.0". The schematic of the experimental setup and data acquisition is shown in Figure 5.4. 


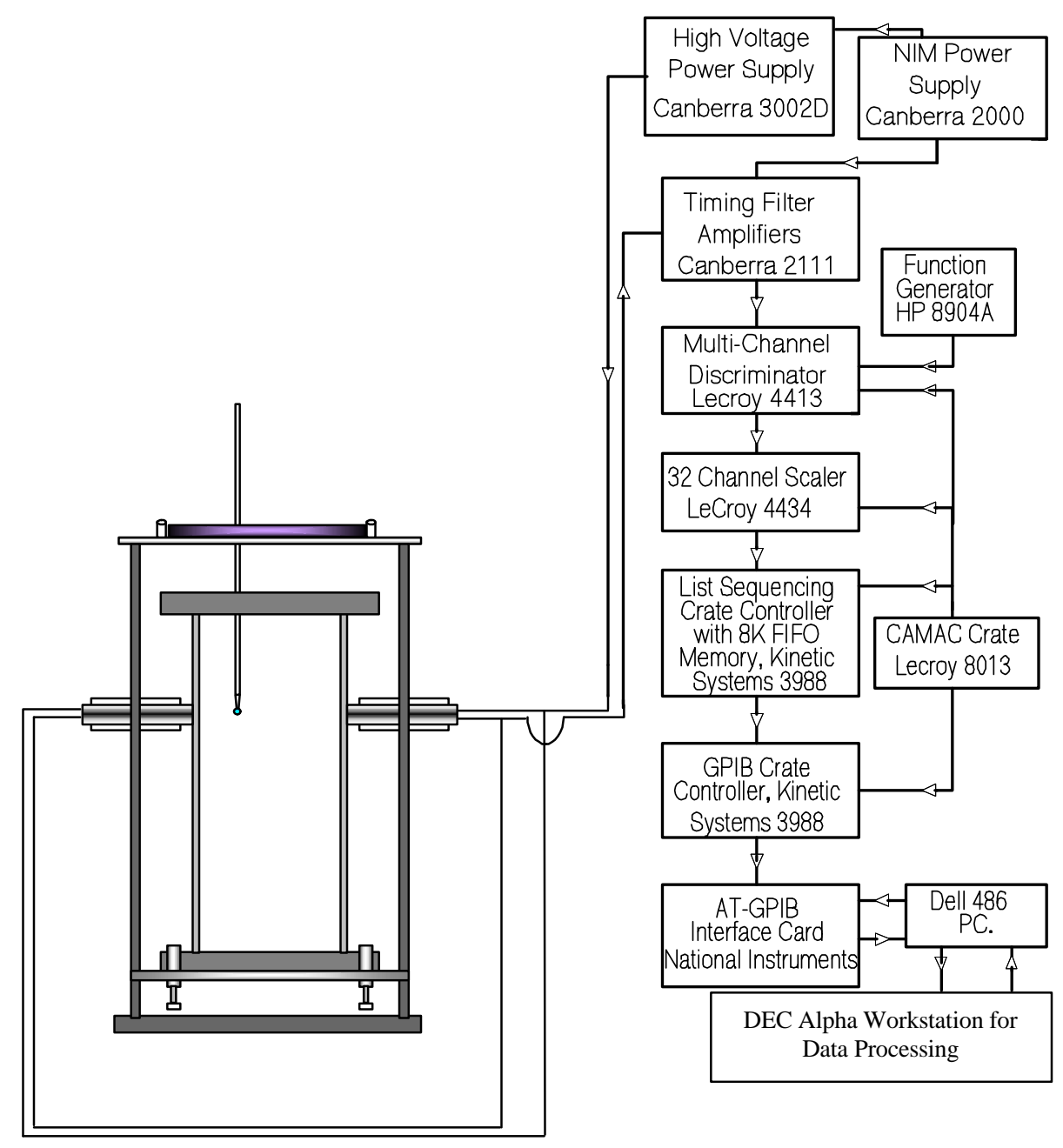

Figure 5.4: Schematic of the Experimental Setup to Verify the Monte Carlo Simulations

A set of four 2"x2" $\mathrm{NaI}(\mathrm{Tl})$ detectors was used for the data acquisition. The detectors were mounted flush to the column at an axial level of $35.4 \mathrm{~cm}$ from the bottom of the column. The detectors were positioned at $90^{\circ}$ degrees to each other (in the plane of the detectors). The total column height used for the reported experiments was $48 \mathrm{~cm}$. experiments were conducted with an empty column, the column filled with water, and with air being sparged into the column filled with water. The objectives of the experiments were twofold. Since the modeled counts belong only to the photopeak portion of the spectrum, the first objective was to determine the correct threshold for the data acquisition system. The second objective was to acquire data at this critical threshold to verify the optimization routines, and to evaluate the particle-position-reconstruction programs against such experimental data. 
Figure 5.5 displays the results of the spectrum analysis for the four detectors used in this study. The radiation counts were acquired by placing the radioactive particle in the center of the column, in the plane of the detectors. It is clear from the figure that a threshold of $300 \mathrm{mV}$ is appropriate as the one signifying the start of the photopeak portion of the spectrum. The $\mathrm{Sc}^{46}$ isotope has two photopeaks at $0.889 \mathrm{MeV}$ and $1.12 \mathrm{MeV}$. Depending on the amplifier gain settings, different threshold scales $(\mathrm{mV})$ map to the same scale of $\gamma$ ray photon energies.

The two photopeaks of the $\mathrm{Sc}^{46}$ isotope are evident from Figure 5.5. Similar analysis was carried out for other locations of the radioactive source. Though the Compton portion of the spectrum showed dependence on the location of the radioactive source, the photopeak portion of the spectrum was almost independent of the location of the source in terms of the start of the photopeaks. With an identified critical threshold of $300 \mathrm{mV}$, the data was acquired with the radioactive particle placed in 27 different locations, at a sampling frequency of $50 \mathrm{~Hz}$ for a total sampling time of 3.84 seconds. These 27 positions were located on 3 planes, one of the planes being the plane of the detectors, with another plane at above and one below of this plane. In each plane, the data was acquired with the particle placed at the center of the column, and at eight other positions located on a circle of radius $5 \mathrm{~cm} 45^{\circ}$ apart. The source strength of the particle used in this study was approximately $95 \mu \mathrm{Ci}$.

Figure 5.6 shows the comparison of the experimentally observed particle position to the one reconstructed by the procedure outlined above. The experiments were conducted with water as the intervening medium. A resolution of $1 \mathrm{~cm}$ was used for generating the 3-D grid over which the particle position is reconstructed. The position reconstruction in the $\mathrm{x}-$ $\mathrm{y}$ plane is satisfactory given the grid that was used. However, the resolution in the zdirection is far from satisfactory. Similar trends were observed in an empty column, as well as in the one with a gas-liquid dispersion. The reason for this observation is straightforward. A set of four detectors is used to resolve the particle position in the $\mathrm{x}-\mathrm{y}$ plane, whereas only one level of detectors are used to resolve the particle position in the zdirection -- hence, the observed inaccuracy is inherent in this experimental design which was utilized only as a quick test of the proposed method. A full-scale data acquisition, with multiple detector-levels has to be accomplished to fully resolve the $\mathrm{z}$-coordinate of the particle position. Nevertheless, these results validate the numerical technique, and further experimentation, along with modifications in the numerical schemes for particle position reconstruction are being evaluated to refine the procedure. 

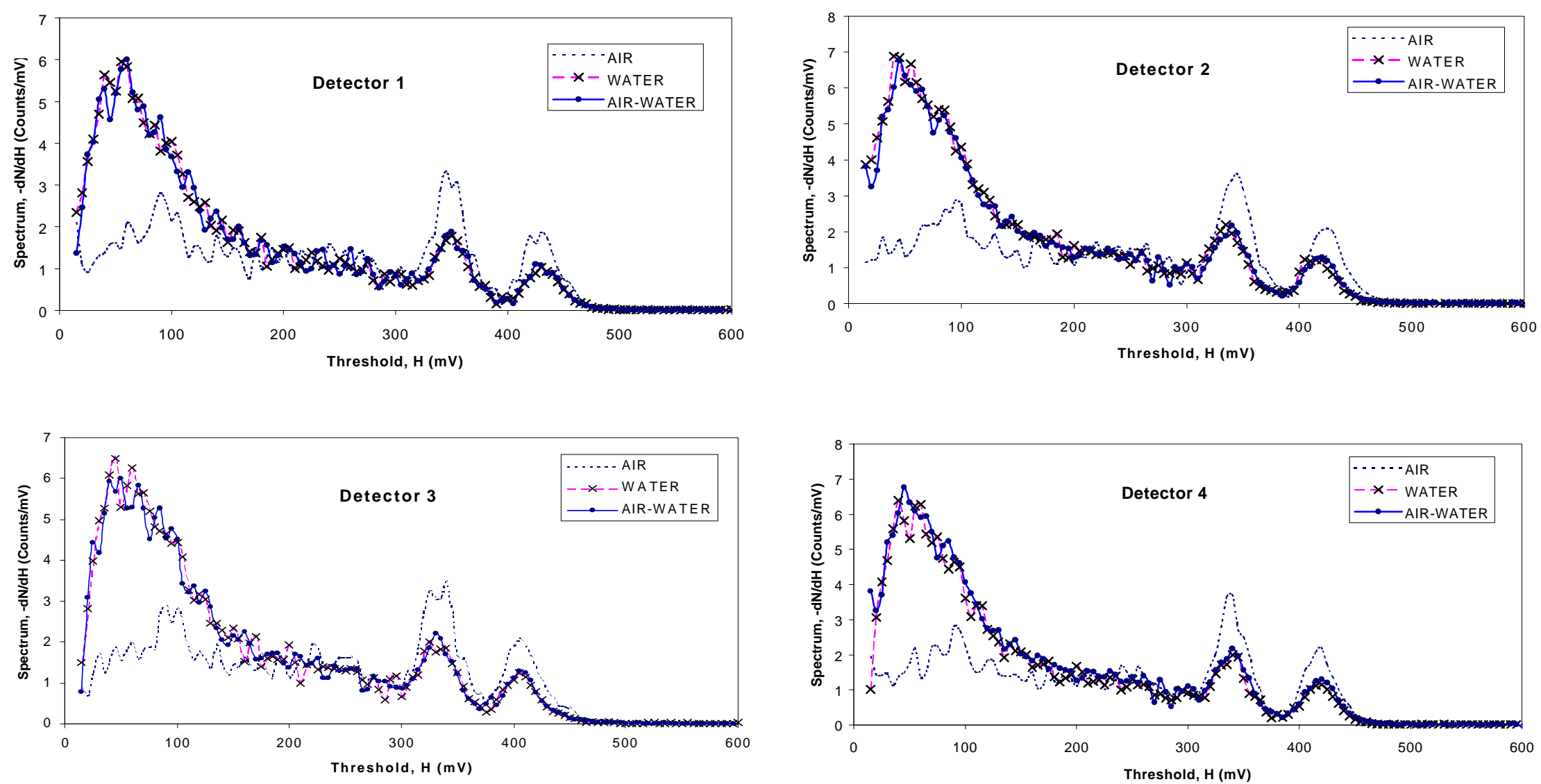

Figure 5.5: $\quad$ Spectrum Analysis of the Four Detectors used in the Experiments for Identification of the Threshold Signifying Beginning of the Photopeak 

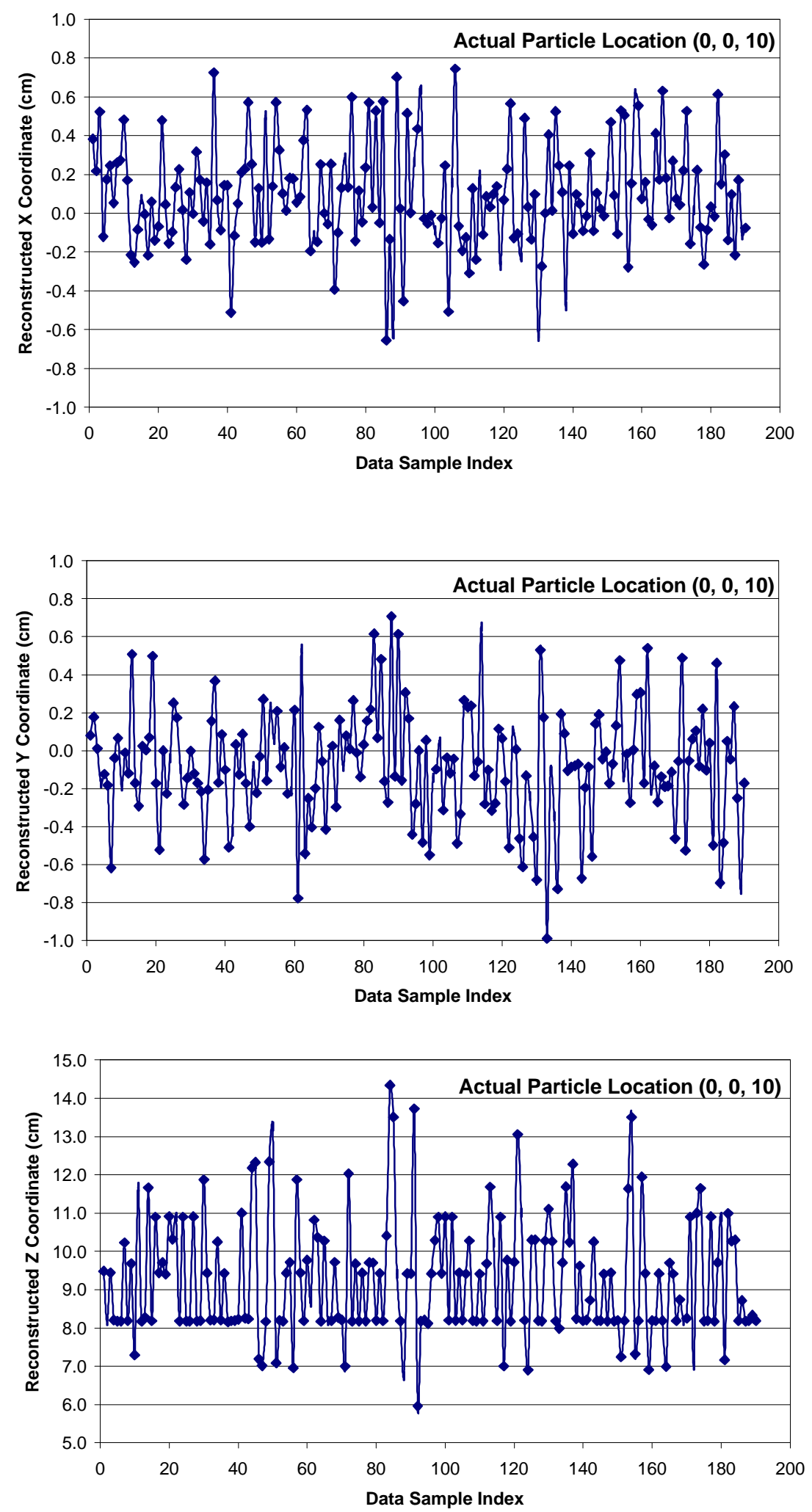

Figure 5.6: Reconstructed Particle Position Over 190 Data Points Acquired Every 20 ms 


\subsection{Conclusions}

The Monte Carlo technique has been validated against experimental data with improved computational efficiency resulting in orders of magnitude reduction in computation times. Further experimentation with more detectors as well as with different data acquisition settings is being carried out to determine the optimal implementation procedure for application to pilot plant scale columns, which are inherently operating with high dispersed phase volume fraction. It should be noted that CARPT is the only technique that can provide information on the velocity fields in large dense opaque systems, where other nonintrusive techniques are entirely inadequate. Hence, our developed Monte Carlo procedure is expected to be used extensively.

\subsection{References}

Beam, G. B., Wielopolski, L., Gardner, R. P. and Verghese, K., 1978, Monte Carlo calculation of efficiencies of right-circular cylindrical NaI detectors for arbitrarily located point sources, Nuclear Instruments and Methods, 154(3), 501-508

Devanathan, N., 1991, Investigation of Liquid Hydrodynamics in Bubble Columns via a Computer Automated Radioactive Particle Tracking (CARPT) Facility, D.Sc. Thesis, Washington University, St. Louis, Missouri

Devanathan, N., Moslemian, D. and Dudukovic, M. P., 1990, Flow Mapping in Bubble Columns Using CARPT, Chem. Engng. Sci., 45, 2285-2291

Dudukovic', M. P., Degaleesan, S., Gupta, P. and Kumar S. B., 1997, Fluid Dynamics in Churn-turbulent bubble columns: Measurements and modeling, American Society of Mechanical Engineers, Fluids Engineering Division (Publication) FED Gas-Liquid Two Phase Flows Proceedings of the 1997 ASME Fluids Engineering Division Summer Meeting, FEDSM'97. Part 16 (of 24) Jun 22-26 1997 v 16, Vancouver, Canada

Gupta, P., 1997, Monte Carlo Applications For Multi-Phase Flow Mapping and Visualization Using CARPT, CREL Annual Report-1997, 111-126

Larachi, F., Kennedy, G. and Chaouki, J., 1994, A $\gamma$-ray detection system for 3-D particle tracking in multiphase reactors, Nuclear Instruments and Methods in Physics Research A., 338, 568-576

Nardi, E., 1970, A note on Monte Carlo calculations in NaI crystals, Nuclear Instruments and Methods, 83, 331-332

Saito, K. and Moriuchi, S., 1981, Monte Carlo calculation of accurate response functions for a $\mathrm{NaI}(\mathrm{Tl})$ detector for gamma rays, Nuclear Instruments and Methods, 185, 299-308

Steyn, J. J., Huang, R. and Harris, D. W., 1973, Monte Carlo calculation of clad NaI(Tl) scintillation crystal response to gamma, Nuclear Instruments and Methods, 107, 465-475 


\section{FLUID DYNAMICS IN HIGH PRESSURE SLURRY BUBBLE COLUMNS}

\subsection{Introduction}

In this work at OSU a study of hydrodynamic characteristics of high-pressure and hightemperature bubble columns were undertaken. The PIV technique was utilized to quantify the rise velocity and interactive dynamics of bubbles. In this report, the flow visualization technique using the PIV's unique particle tracking algorithm and blob size analysis is used to investigate the single bubble rise velocity and the bubble size distribution in a highpressure bubble column. Furthermore, a computational model for gas-liquid-solid fluidization systems and a two-dimensional code based on it are developed in this study. In this model, the volume-averaged method, the dispersed particle method and the volumeof-fluid method are used, respectively, to account for the flow of liquid, solid, and gas phases. The gas-liquid interfacial mass, momentum, and energy transfer is described by a continuum surface force model. A collision model, which includes the particle-fluid and particle-particle interactions during the entire process of collision, is considered in the simulation. The particle-bubble interaction is formulated by incorporating the surface tension force in the equation of motion of the particles. The simulation of a single bubble rising in a liquid-solid fluidized bed and the particle entrainment with an emerging bubble is conducted and the results are in good agreement with the experimental findings.

\subsection{Experimental Set-up}

The high-pressure and high-temperature bubble column system used in this study consists of a vertical column, a liquid supply tank, a liquid exhaust reservoir, a piston pump, and a pulsation damper. The vertical column comprises three sections: the plenum, the test, and the disengagement sections. The pipes and column are insulated. Two sizes of stainless steel columns are used: one is $50.8 \mathrm{~mm}$ in diameter and $0.80 \mathrm{~m}$ in height and the other is $101.6 \mathrm{~mm}$ in diameter and $1.58 \mathrm{~m}$ in height. The latter is specifically used for the bubble rise velocity measurement. In both columns, there are three pairs of planar quartz windows with dimensions of $12.7 \mathrm{~mm}$ in width and $92 \mathrm{~mm}$ in height. These windows allow viewing through the entire test section of the column. The plane shape circumvents optical distortion in photography.

Nitrogen is used as the gas phase and as the pressurizing source. The inlet pressure is regulated by a two-stage regulator, and the flow rate is controlled by a flow control valve. To maintain a constant system temperature, nitrogen is preheated by a gas heater to the desired temperature before being introduced into the column. Nitrogen enters the bubble column through a multi-orifice sparger in a ring arrangement with an orifice diameter of 3 $\mathrm{mm}$. After exiting the bubble column, the gas passes through a back pressure regulator, which is used to control the pressure of the column. It is then ventilated into the atmosphere through the demister and the cooling tank. The gas flow rate is measured with either an electromagnetic gas flow meter or a rotameter at the end of the gas exhaust line. Paratherm NF heat transfer fluid, a stable organic liquid $\left(T_{b}=327^{\circ} \mathrm{C}\right.$ at $\left.0.1 \mathrm{MPa}\right)$, is used as the liquid phase. The liquid is operated in a batch mode. The liquid level in the column is maintained near the top of the column so that foaming will not affect the global-scale measurements. 
A high-speed PIV system developed in-house is used to analyze the characteristics of bubbles. The CCD image picked up on the high-speed camera features 765 pixels across by 246 lines. The framing rate of the camera can be selected up to 480 fields/sec. A frame grabber, which is equipped with $40 \mathrm{MHz}$ maximum pixel clock to support the highframing rate, simultaneously digitizes the CCD image from the camera. Further, the highspeed camera is connected to the high-speed video recorder to store the images for further studies. The PIV technique used here relies on a particle-tracking algorithm (Chen and Fan, 1992) to determine the velocity fields of the particles. The particle-tracking algorithm involves matching the same objects in three or more consecutive fields.

\subsection{Single Bubble Rise Velocity}

The rise velocity, $U_{b}$, of single bubbles of known sizes is measured at various pressures ranging from 0.1 to $19.4 \mathrm{MPa}$ for three temperatures, 27,47 and $78^{\circ} \mathrm{C}$. The bubble size is represented by the equivalent spherical diameter, $d_{e}$. The results are shown in Figure 6.1 for $27^{\circ} \mathrm{C}$ and $78^{\circ} \mathrm{C}$. As shown in the figure, for a given bubble size, $U_{b}$ tends to decrease with increasing pressure at both temperatures. To elucidate the effects of pressure and temperature, or more directly, the effects of physical properties of the gas and liquid phases on the variation of $U_{b}$ with $d_{e}$, the following three predictive equations are considered for comparisons.

1) The Mendelson (1967) equation recently modified by Maneri (1995),

$U_{b}=\left[\frac{2 c \sigma}{\rho_{l} d_{e}}+\left(\frac{\Delta \rho}{\rho_{l}}\right) \frac{g d_{e}}{2}\right]^{1 / 2}$

is applicable for both single gas bubbles and liquid drops rising/falling in a continuous phase of purified, low-viscosity liquids (immiscible with the drop liquid).

2) The Fan-Tsuchiya (1990) correlation, generalized for high-pressure systems, has a dimensionless form

$U_{b}^{\prime}=U_{b}\left(\frac{\rho_{I}}{\sigma g}\right)^{1 / 4}=\left\{\left[\frac{M 0^{-1 / 4}}{K_{b}}\left(\frac{\Delta \rho}{\rho_{l}}\right)^{5 / 4} d_{e}^{\prime 2}\right]^{-n}+\left[\frac{2 c}{d_{e}^{\prime}}+\left(\frac{\Delta \rho}{\rho_{l}}\right) \frac{d_{e}^{\prime}}{2}\right]^{-n / 2}\right\}^{-1 / n}$

where the dimensionless bubble diameter is given by

$d_{e}^{\prime}=d_{e}\left(\rho_{1} g / \sigma\right)^{1 / 2}$

Three empirical parameters, $n, c$, and $K_{b}$, in Eq. (2) reflect three specific factors governing the rate of bubble rise. They are the contamination level of the liquid phase, variation due to dynamic effects of the surface tension with mono- or multicomponent liquids, and the viscous nature of the surrounding medium. The suggested values of these parameters are

$n= \begin{cases}0.8 & \text { for contaminated liquids } \\ 1.6 & \text { for purified liquids }\end{cases}$ 
$c= \begin{cases}1.2 & \text { for monocomponent liquids } \\ 1.4 & \text { for multicomponent liquids }\end{cases}$

$\mathrm{K}_{\mathrm{b}}=\max \left(\mathrm{K}_{\mathrm{b} 0} \mathrm{M} \mathrm{O}^{-0.038}, 12\right)$

where $K_{b 0}= \begin{cases}14.7 & \text { for aqueous solutions } \\ 10.2 & \text { for organic solvents/mixtures }\end{cases}$

3) The Tomiyama et al. (1995) correlation, which is given in terms of drag coefficient $C_{D}=\frac{4}{3} g \Delta \rho d_{e} /\left(\rho_{l} U_{b}^{2}\right)$, consists of three equations, depending on the system purity:

$C_{D}=\max \left\{\min \left[\frac{16}{R e}\left(1+0.15 R e^{0.687}\right), \frac{48}{R e}\right], \frac{8}{3} \frac{E_{0}}{E 0+4}\right\}$

for purified systems;

$C_{D}=\max \left\{\min \left[\frac{24}{R e}\left(1+0.15 R e^{0.687}\right), \frac{72}{R e}\right], \frac{8}{3} \frac{E_{0}}{E 0+4}\right\}$

for partially contaminated systems; and

$$
C_{D}=\max \left[\frac{24}{R e}\left(1+0.15 R e^{0.687}\right), \frac{8}{3} \frac{E o}{E o+4}\right]
$$

for sufficiently contaminated systems.

In the above equations, i.e., Eqs. (1), (2), and (5), the dimensionless groups are defined as

$M o=\frac{g \Delta \rho \mu_{l}^{4}}{\rho_{l}^{2} \sigma^{3}}$,

$\operatorname{Re}=\frac{d_{e} U_{b} \rho_{l}}{\mu_{l}}$, and

$E o=\frac{g \Delta \rho d_{e}^{2}}{\sigma}$

where $\Delta \rho=\rho_{1}-\rho_{g}$. It is noted that $U_{b}$ can be obtained explicitly from Eq. (1) or (2) for a given $d_{e}$ as well as gas and liquid physical properties, while it can only be obtained implicitly from Eq. (5).

Figure 6.1 shows the predictions based on Eqs. (1), (2), and (5). For predictions, directly measured values of physical properties under various operating pressures and temperatures are used. Since the Mendelson equation, Eq. (1), is valid only under inviscid conditions, the limited agreement between the measured and calculated results at the low 
temperature (Figure 6.1a) suggests that viscous forces predominate in the bubble rise process. On the other hand, at the high temperature (Figure 6.1b), there is a strong agreement over the bubble size range $d_{e}>2 \mathrm{~mm}$ including the sharp breakpoint/peak. This indicates that at high temperature the liquid used in this study tends to behave as a pure inviscid liquid. Note that over the pressure range from 0.1 to $19.4 \mathrm{MPa}$, the liquid viscosity varies from 29 to $48 \mathrm{mPa}$ s at $27^{\circ} \mathrm{C}$, whereas it is almost constant within a range from 4.7 to $5.2 \mathrm{mPa}$ s at $78^{\circ} \mathrm{C}$.

The Fan-Tsuchiya (1990) correlation applied for the present liquid (Eq. (2) with $n=1.6, c$ $=1.4, K_{b 0}=10.2$ ) demonstrates good overall predictive capability except for the high temperature case, which exhibits a sharp peak near $d_{e}=2 \mathrm{~mm}$ (see Figure 6.1b). The Tomiyama et al. (1995) equation, Eq. (5a), also has good general applicability and predicts the high temperature $d_{e}=2 \mathrm{~mm}$ peak; however, it tends to underestimate the $U_{b}$ values over the rest of $d_{e}$ range.

The consistent difference in $U_{b}$ prevailing between 0.1 and $19.4 \mathrm{MPa}$ for $d_{e}>2 \mathrm{~mm}$ is due to the significant increase in gas density (as large as 200-fold increase with pressure from 0.1 to $19.4 \mathrm{MPa}$ ). The density effect is accounted for in Eqs. (1) and (2) in terms of $\Delta \rho / \rho_{1}$ or in Eq. (5) in terms of both $\Delta \rho / \rho_{l}$ and Eo. As can be seen from the equations and figure, the density difference between the continuous liquid phase and the dispersed gas phase plays an important role in determining $U_{b}$, especially for large bubbles.

Figure 6.2 shows the $R e-E o$ relationship often utilized in representing the general rise characteristics of single bubbles in liquids (Clift et al., 1978; Bhaga and Weber, 1981). The thin background lines signify the general, quantitative trend for the rise velocity of single bubbles in purified Newtonian liquids under ambient conditions, plotted with constant intervals of $\log M o$. The figure shows the general agreement in prediction between the Fan-Tsuchiya (1990) correlation (Eq. (2); solid lines) and the Tomiyama et al. (1995) equation (Eq. (5a); dot-dash lines). The present results under four conditions are plotted in the figure, along with the corresponding prediction based on Eq. (2) given by the thick solid lines. By employing accurate values for physical properties of the liquid phase and the gas density at given pressures and temperatures for Eq. (2), the experimental results can be successfully represented over the entire Eo range (i.e., bubble size range) by Eq. (2) as shown in Figure 6.2. 

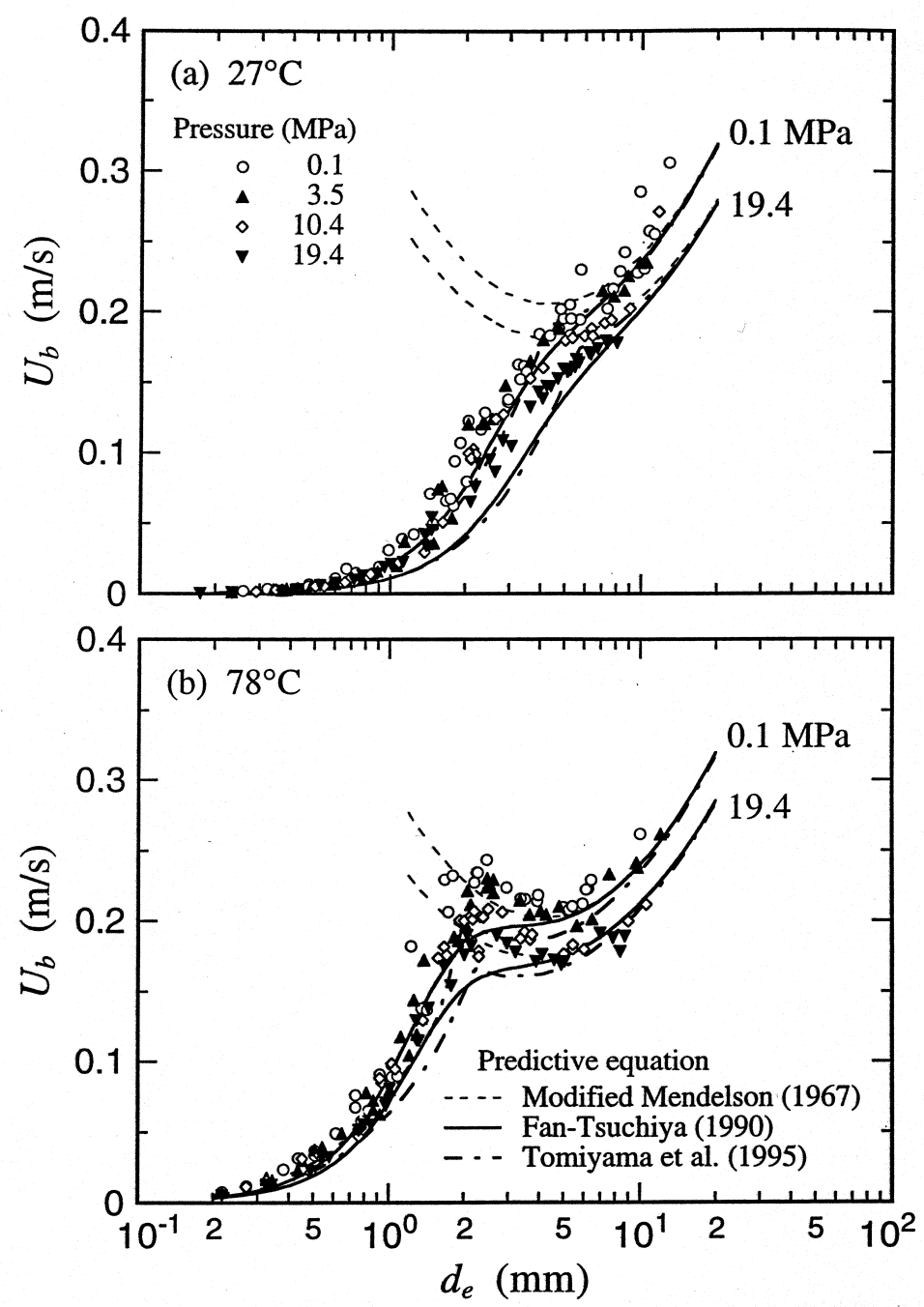

Figure 6.1. Effect of Pressure on Terminal Rise Velocity of Single Bubbles in Paratherm NF Heat Transfer Fluid and Predicted Values at (a) $27^{\circ} \mathrm{C}$ and (b) $78^{\circ} \mathrm{C}$ 


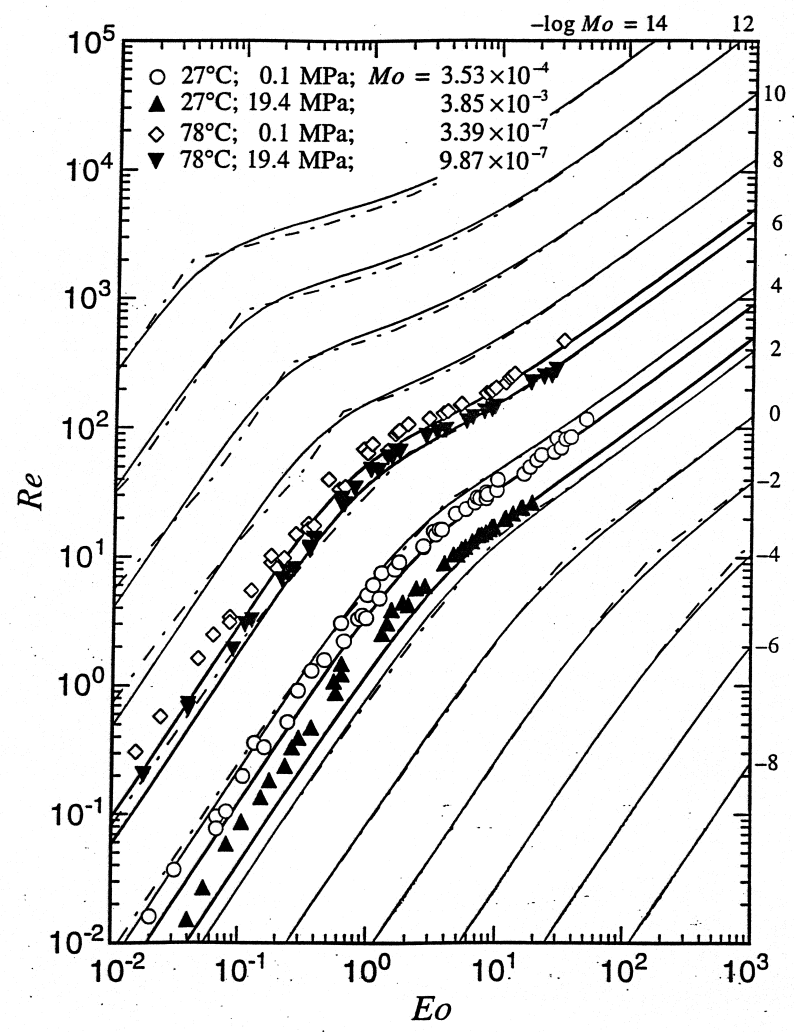

Figure 6.2. Comparisons of Measured and Calculated $R e$ of Single Bubbles in Paratherm NF Heat Transfer Fluid Under Varied Pressure and Temperature Conditions. The Fan-Tsuchiya (1990) and Tomiyama et al. (1995) Relations are Plotted (_ and - - - , respectively) at Regular Intervals of Mo Values. The Fan-Tsuchiya (1990) Correlation at Measured Mo Values for Comparison With Measured Re-Eo data ( ）

\subsection{Bubble Size Distribution}

In a bubble column, bubbles undergo interactions; they can be weak as in hindered rising or strong as in coalescence and breakup. Under the hindered rising condition, the inherent rise characteristics of single bubbles play an essential role, and the initial/detached bubble size during bubble formation as well as the number density of dispersed bubbles determines the rise velocity and residence time of the bubbles in the column. For highly interactive bubbles, the dominant factor controlling their dispersion state is their dynamic size variation due to local bubble coalescence and breakup. The bubble size distribution is evaluated through direct video enhanced visualization of bubbles at a location $0.40 \mathrm{~m}$ above the gas distributor for various pressures under three different temperature and superficial gas velocity conditions: $27^{\circ} \mathrm{C}$ and $8 \mathrm{~cm} / \mathrm{s} ; 27^{\circ} \mathrm{C}$ and $2 \mathrm{~cm} / \mathrm{s}$; and $78^{\circ} \mathrm{C}$ and 5 $\mathrm{cm} / \mathrm{s}$.

As pressure increases from 0.1 to $15.2 \mathrm{MPa}$, large bubbles gradually disappear. The corresponding distributions of bubble size, $d_{b}$, from 0.6 to $4.3 \mathrm{~mm}$, are shown in Figure 6.3. The shift in bubble size distribution demonstrates the effect of pressure on bubble size. 
It can be seen from the figure that for pressures between 0.1 and $3.5 \mathrm{MPa}$, the bubble size is widely distributed with the fraction of bubbles of size larger than $2.5 \mathrm{~mm}$ decreasing noticeably with increasing pressure. For pressures greater than $7.0 \mathrm{MPa}$, the size distribution is appreciably narrower. As the pressure increases from 0.1 to $15.2 \mathrm{MPa}$, the dominant bubble size shifts from 2.5 to $0.6 \mathrm{~mm}$, and the fraction of bubble sizes between 2 and $4 \mathrm{~mm}$ decreases by three quarters.
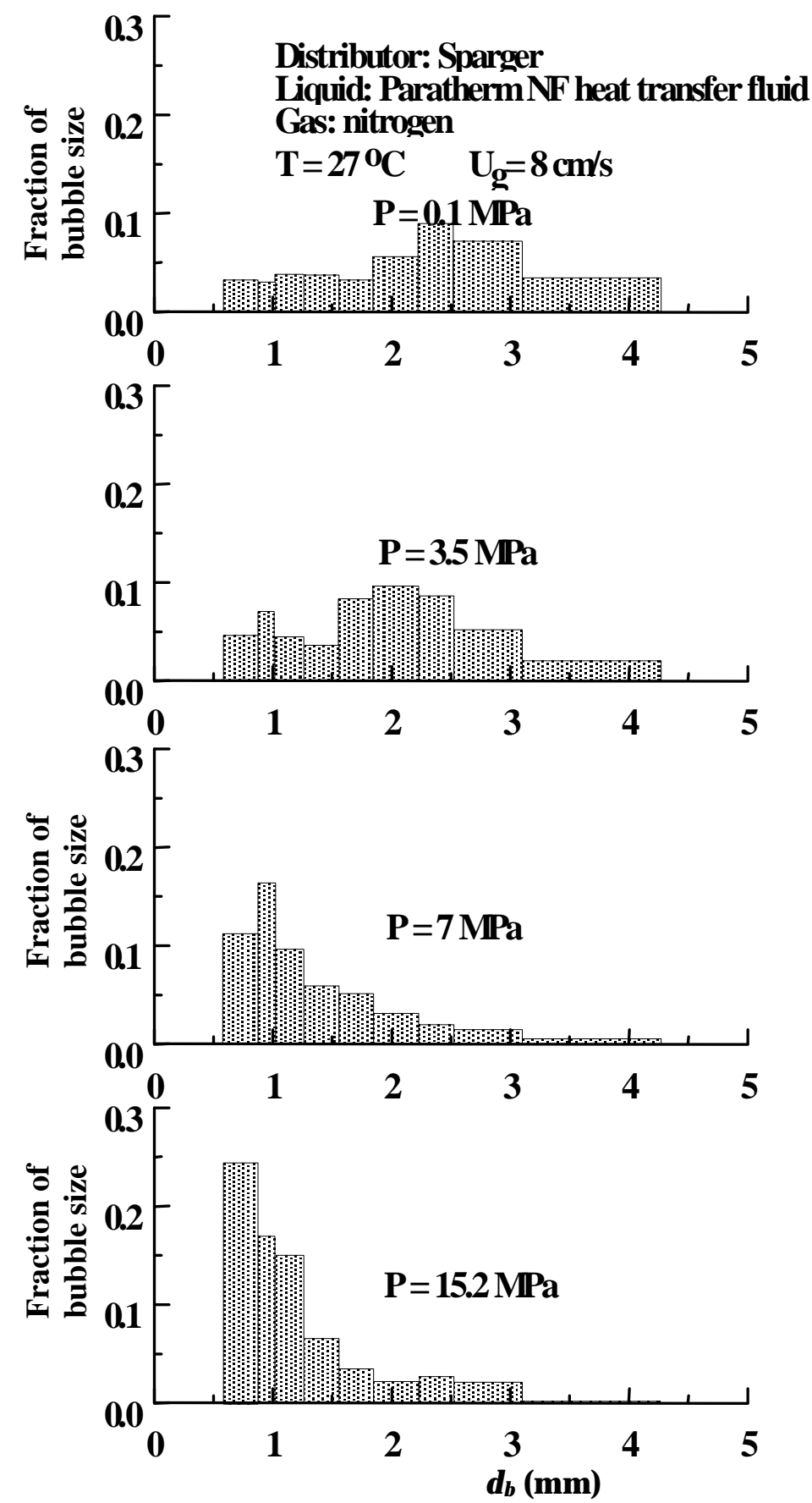

Figure 6.3. Bubble Size Distributions Under Various Pressures at $T=27^{\circ} \mathrm{C}$ and $U_{g}=8$ $\mathrm{cm} / \mathrm{s}$ 
Figure 6.4 shows the associated bubble size distributions for different pressures at $27^{\circ} \mathrm{C}$ and $U_{g}=2 \mathrm{~cm} / \mathrm{s}$. Again, the general trend is clearly shown that large bubbles disappear progressively with increasing pressure under both conditions. Further, the fraction of large bubbles is less at the gas superficial velocity of $2-\mathrm{cm} / \mathrm{s}$ case relative to the fraction of large bubbles at the gas superficial velocity of $8-\mathrm{cm} / \mathrm{s}$, especially for lower pressures.
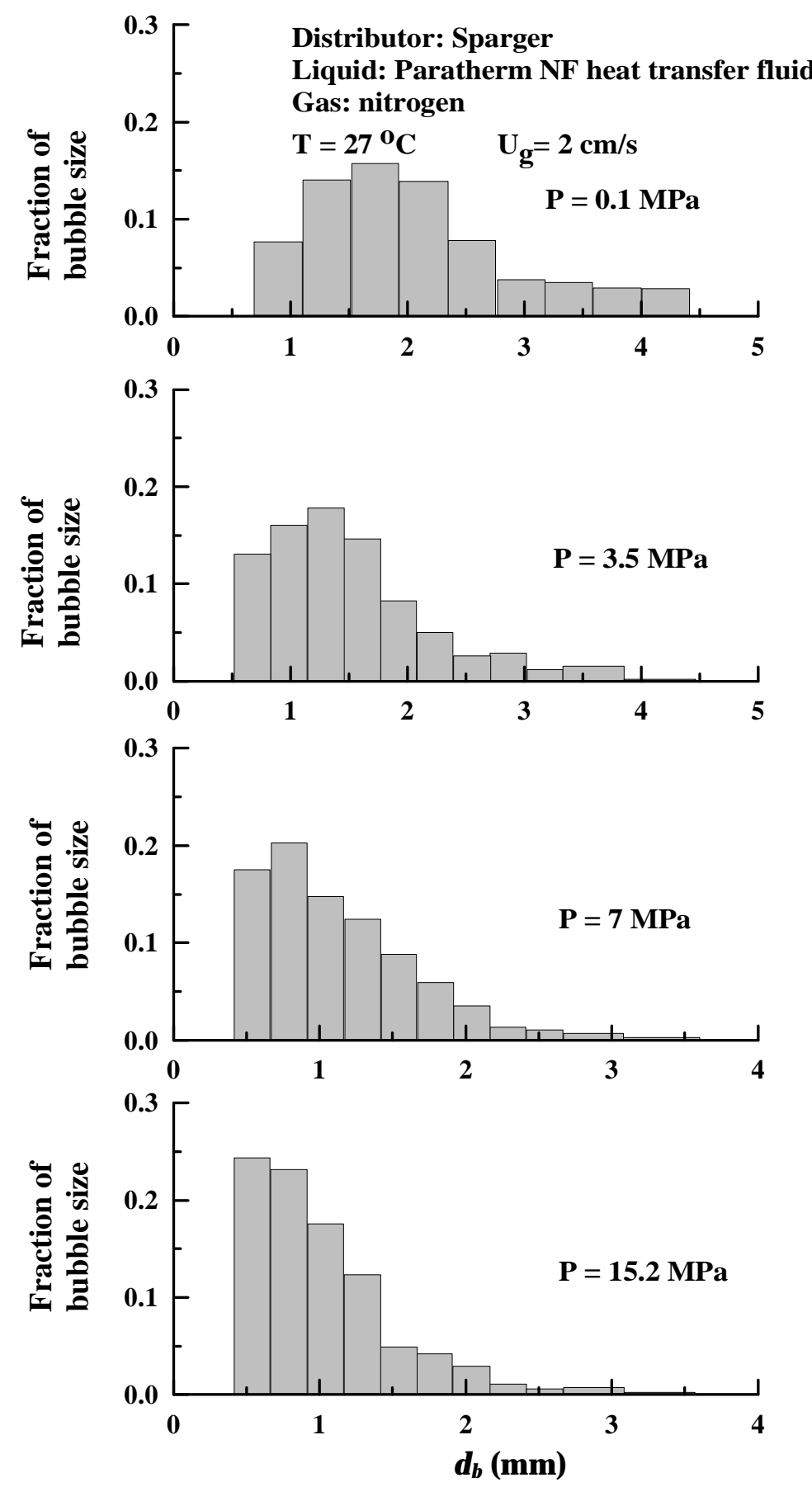

Figure 6.4. Bubble Size Distributions Under Various Pressures at $T=27^{\circ} \mathrm{C}$ and $U_{g}=2$ $\mathrm{cm} / \mathrm{s}$ 
Figure 6.5 shows the corresponding bubble size distributions at $78^{\circ} \mathrm{C}$ and $U_{g}=5 \mathrm{~cm} / \mathrm{s}$. As can be seen from the figure, although the most prevalent bubble size $(0.5-0.6 \mathrm{~mm})$ is the same for all pressures, the fraction of bubbles of size larger than $2 \mathrm{~mm}$ is reduced significantly as the pressure increases from 0.1 to $3.5 \mathrm{MPa}$. A comparison between Figures 6.3 and 6.5 clearly shows that, at a pressure of $3.5 \mathrm{MPa}$, the dominant bubble size is reduced from $2 \mathrm{~mm}$ at $27^{\circ} \mathrm{C}$ to $0.5 \mathrm{~mm}$ at $78^{\circ} \mathrm{C}$.
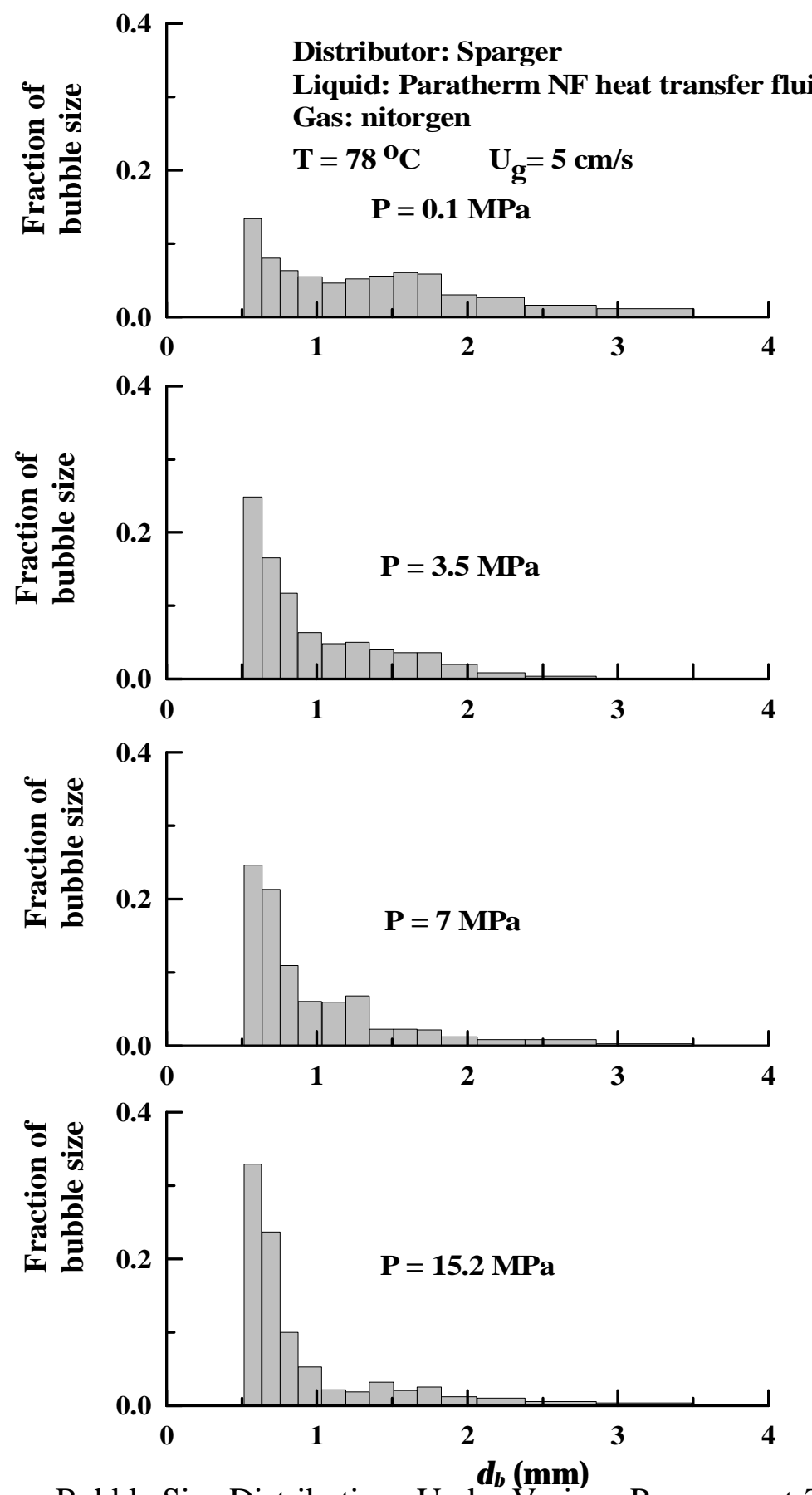

Figure 6.5. Bubble Size Distributions Under Various Pressures at $T=78^{\circ} \mathrm{C}$ and $U_{g}=5$ $\mathrm{cm} / \mathrm{s}$ 


\subsection{Theoretical Model and Numerical Method}

\subsubsection{Liquid-Phase Model}

For fluidization systems, Anderson and Jackson (1967) developed the volume-averaged continuity and momentum equations. The original equations apply to both discrete and continuous phases. For the gas-liquid-solid systems, an extension of their model can lead to the volume-averaged equations for the three individual phases. In this study, the motion of particles is determined by the discrete particle method and the motion of gas bubbles is computed by a front tracking method. Thus, the averaged equations are applied only to the liquid phase.

The continuity equation can be given as

$$
\frac{\partial \varepsilon_{1}}{\partial \mathrm{t}}+\nabla \cdot\left(\varepsilon_{1} \mathrm{v}\right)=0
$$

and the momentum equation is

$$
\rho_{\mid} \frac{\partial\left(\varepsilon_{\mid} v\right)}{\partial t}+\rho_{\mid} \nabla \cdot\left(\varepsilon_{\mid} \mathrm{vv}\right)=-\varepsilon_{\mid} \nabla p+\varepsilon_{\mid} \nabla \cdot \tau+\varepsilon_{\mid} \rho_{\mid} g+f_{b}
$$

where $v$ is liquid velocity vector, $\varepsilon_{\mid}$is liquid holdup, $\rho_{\mid}$is liquid density, $p$ is scalar pressure, $\tau$ is viscous stress tensor, $g$ is gravity acceleration, and $f_{b}$ is total volumetric body force acting on the liquid phase other than gravity force.

The Newtonian viscous stress tensor is used which is given as

$\tau=2 \mu S=\mu\left[(\nabla v)+(\nabla v)^{\top}\right]$

where $S$ is the rate-of strain tensor and $\mu$ is the kinematic viscosity coefficient.

\subsubsection{Gas Phase Model}

The gas phase is present in the form of bubbles. The flow inside the gas bubble is governed by single-phase Navier-Stokes equations. Due to the significant density difference between the gas and the liquid-solid suspension, the momentum transfer from the gas inside the bubble to the suspension is negligible except at the gas-liquid interface where the surface tension force across the interface acts on the liquid from the gas phase. Therefore, a gas bubble can be treated as a void with the motion and the topological change of its surface governed by the liquid-solid flow and the interface dynamics (Hirt and Nichols, 1981). The free surface of the gas void is reconstructed by a scalar field $\alpha(x, t)$, where $\alpha(x, t)=1$ in the liquid or liquid-solid mixture, $0<\alpha(x, t)<1$ at the free surface, and $\alpha(x, t)=0$ in the void. The advection equation for $\alpha(x, t)$ is

$$
\frac{\partial \alpha}{\partial \mathrm{t}}+(\mathrm{v} \cdot \nabla) \alpha=0
$$


In the gas-liquid free surfaces, the stress boundary condition follows Laplace's equation as

$$
p_{s}=p-p_{v}=\sigma \kappa
$$

where the surface pressure $p_{\mathrm{s}}$ is the surface tension-induced pressure jump across a fluid interface. The continuum surface force (CSF) model (Brackbill et al., 1992) converts the surface force into a volume force within free surfaces. The volume force at the free surfaces is given by the CSF model as

$$
\mathrm{f}_{\mathrm{sv}}(\mathrm{x}, t)=\sigma \kappa(\mathrm{x}, t) \nabla \alpha(\mathrm{x}, t) .
$$

This volume force is added to the volumetric body force term, $f_{b}$ in the momentum equation at the free surfaces.

\subsubsection{Dispersed Particle Model}

The motion of a particle in a flow field can be described in the Lagrangian coordinates with its origin attached to the center of a moving particle. The motion of a single particle can be described by its acceleration and rotation in a non-uniform flow field. The particle accelerating in the liquid is governed by Newton's second law of motion as

$$
\mathrm{m}_{\mathrm{p}} \frac{\mathrm{dv}{ }_{\boldsymbol{p}}}{\mathrm{dt}}=\mathrm{F}_{\text {total }} \text {. }
$$

The forces acting on a particle include interface forces between the fluid and the particle, and forces imposed by external fields. The total force acting on a particle is composed of all applicable forces, including drag, pressure gradient, added mass, gravity/buoyancy, Magnus force, Basset force, and others,

$$
F_{\text {total }}=F_{D}+F_{P}+F_{A M}+F_{G / B}+\sum_{i} F_{i}
$$

The drag force acting on a suspended particle is proportional to the relative velocity between the phases and has the following form:

$$
F_{D}=\frac{1}{2} C_{D} \rho A\left|v-v_{p}\right|\left(v-v_{p}\right)
$$

where $A$ is the cross-sectional area of the particle to the direction of the incoming flow, $C_{D}$ is the drag coefficient, which is a function of the particle Reynolds number, $R_{p}$. For rigid spherical particles, the drag coefficient $C_{D}$ can be estimated by the following equations (Rowe and Henwood, 1961):

$$
C_{D}=\left\{\begin{array}{l}
\frac{24}{R e_{p}}\left(1+0.15 R e_{p}^{0.687}\right), R e_{p}<1000 \\
0.44, R e_{p} \geq 1000
\end{array}\right.
$$


In the liquid-solid suspension, the drag force depends strongly on the local liquid holdup in the vicinity of the particle under consideration. The effective drag coefficient can be obtained by the product of the drag coefficient for an isolated particle and a correction factor as given by (Wen and $\mathrm{Yu}, 1966$ )

$C_{D}^{*}=C_{D} \varepsilon_{1}^{-4.7}$

The added mass force accounts for the resistance of the fluid mass that is moving at the same acceleration as the particle. For a spherical particle, the volume of the added mass is equal to one-half of the particle volume, $V_{p}$, so that

$$
F_{\mathrm{AM}}=\frac{1}{2} \rho_{\mathrm{p}} \mathrm{V}_{\mathrm{p}} \frac{\mathrm{d}}{\mathrm{dt}}\left(\mathrm{v}-\mathrm{v}_{\mathrm{p}}\right)
$$

The sum of the gravity/buoyancy force and the pressure gradient force is given as

$$
\mathrm{F}_{\mathrm{G} / \mathrm{B}}+\mathrm{F}_{\mathrm{P}}=\left(\rho_{\mathrm{p}}-\rho_{\mathrm{I}}\right) \mathrm{V}_{\mathrm{p}} \mathrm{g}-V_{p} \nabla \mathrm{p}
$$

The general scheme of a stepwise molecular dynamic (MD) simulation (Allen and Tildesley, 1987), based on a predictor-corrector algorithm, is used to compute the particle motion. The hard sphere approach is used for the collision dynamics. The normal velocity and momentum changes of colliding particles are determined by a collinear collision model developed by Zhang et al. (1998), which includes the detailed particle-fluid and particleparticle interactions during the entire process of particle collision. In this study, the tangential velocity and momentum changes are formulated and calculated based on a sticking/sliding model.

\subsubsection{Coupling Among Individual Phases}

When particles move into the gas-liquid interface, i.e., $0.5<\alpha(x, t)<1$, the surface tension force is also acting on the particle. This force equals the volumetric surface tension force $f_{s v}$ of Eq. (12) multiplied by the particle volume. If the total force of the particle is larger than the surface tension force, the particle would penetrate the bubble surface. The penetrating particle breaks the bubble surface momentarily upon contact. If the penetrating particle is small, the bubble may recover its original shape upon particle penetration (Chen and Fan, 1989). However, if there are several particles colliding with the bubble surface simultaneously, the resulting force may cause bubble breakage.

Based on Newton's third law of motion, the total forces acting on particles yield a reaction force on the liquid. Therefore, the momentum transfer from particles to liquid is taken into account by adding the volumetric liquid-particle interaction force to the body force term, $f_{b}$, in Eq. (8).

The liquid holdup, $\varepsilon_{\mid}$, is obtained by subtracting the volume fraction of the particles in the computational cell. A pseudo-three dimensional approach, which assumes a thickness equal to the diameter of a spherical particle for the computational cell, is used in calculating the liquid-phase holdup. The liquid properties on the particle surface are obtained by an area-weighted averaging based on the properties at the four grid points of 
the computational cell containing the particle. It is found in this study that the cell averaged liquid holdup can not represent well the phase holdup surrounding a particle when the particle is within an area that has significant solids concentration variations. Therefore, the cell averaged liquid holdup is only used for solving the volume-averaged equations of the liquid phase. When using Eq. (17) to calculate the particle drag coefficient in the liquid-solid medium, the liquid holdup is obtained on the basis of a particle-centered area averaging method.

\subsection{Results From Numerical Study}

\subsubsection{Bubble Rising in Liquid-Solid Fluidized Medium}

A comparison of the simulation and the experimental results obtained for a single bubble rising in a liquid-solid fluidized bed is shown in Figure 6.6. The simulation domain is $3 \times 8$ $\mathrm{cm}^{2}$. One thousand particles with a density of $2,500 \mathrm{~kg} / \mathrm{m}^{3}$ and a radius of $0.5 \mathrm{~mm}$ are used as the solid phase. An aqueous glycerin solution (80 wt\%) with a density of $1,206 \mathrm{~kg} / \mathrm{m}^{3}$, a viscosity of $5.29 \times 10^{-2} \mathrm{~kg} / \mathrm{m} \cdot \mathrm{s}$ and a surface tension coefficient of $6.29 \times 10^{-2} \mathrm{~N} / \mathrm{m}$ is used as the liquid phase. A spherical bubble with a diameter of $1.0 \mathrm{~cm}$ is initially imposed in the computational domain with its center $1.5 \mathrm{~cm}$ above the bottom. Initially, the particles are randomly positioned in a $3 \times 24 \mathrm{~cm}^{2}$ area. Then, the simulation is performed for the particle settling at a liquid velocity of $0.5 \mathrm{~cm} / \mathrm{s}$. At this stage, the bubble is treated as an obstacle and fixed in the original place. An equilibrium bed height is reached at $8 \mathrm{~cm}$, which gives the three-dimensional equivalent solid holdups of 0.44 . With the particles in the equilibrium position, the simulation is restarted with bubble tracking and particle movement. The time step of simulation for liquid and solid phases is $5 \times 10^{-6}$ second. Experiments are performed in a two-dimensional column with a thickness of $7.0 \mathrm{~cm}$. The solids holdup, liquid velocity, and the liquid and solid properties are same as the simulation conditions. The time difference between two frames in Figure 6.6 is 0.1 second. As shown in the figure, the simulation and experimental results of the bubble rising velocity and the bubble shape generally agree well.

\subsubsection{Particle Entrainment}

When bubbles disengage from the bed surface, particle entrainment takes place. By closely following the evolution of the particle flow around a single bubble, the mechanisms of the particle entrainment in the three-phase fluidized bed are studied experimentally by Miyahara et al. (1989), Fan and Tsuchiya (1990) and Tsuchiya et al. (1992). Their studies indicate that particles are drawn from the upper surface of the fluidized bed into the freeboard in the wake behind the bubble, and vortices containing particles are shed from the wake in the freeboard. In this study, a bubble emerging from the liquid-solid fluidized bed is simulated. The solid particles used in the simulation are glass beads with a density of $2,500 \mathrm{~kg} / \mathrm{cm}^{3}$ and a radius of $0.35 \mathrm{~mm}$. The computational domain is $6 \times 12 \mathrm{~cm}^{2}$. Water is used as the liquid phase. The liquid velocity is set at $7.5 \mathrm{~cm} / \mathrm{s}$. The equilibrium bed height is $6.5 \mathrm{~cm}$ and the resulting solid holdup of the fluidized bed equals 0.13 . A spherical bubble with a diameter of $0.8 \mathrm{~cm}$ is initially imposed at $1.5 \mathrm{~cm}$ above the bottom. Figure 6.7 shows the bubble emerging from the bed surface in four frames of the simulation with a time difference of 0.03 second. Frame 1 of Figure 6.7 shows the bubble emerging from 
the upper free surface of the fluidized bed. A group of particles are dragged by the bubble wake in the subsequent frames. An agreement in spatial and temporal variations of the solid particle in the entrainment process with the rising bubble is found between the simulation and the experimental results in pictures by Miyahara et al. (1989) and in sketches by Tsuchiya et al. (1992).
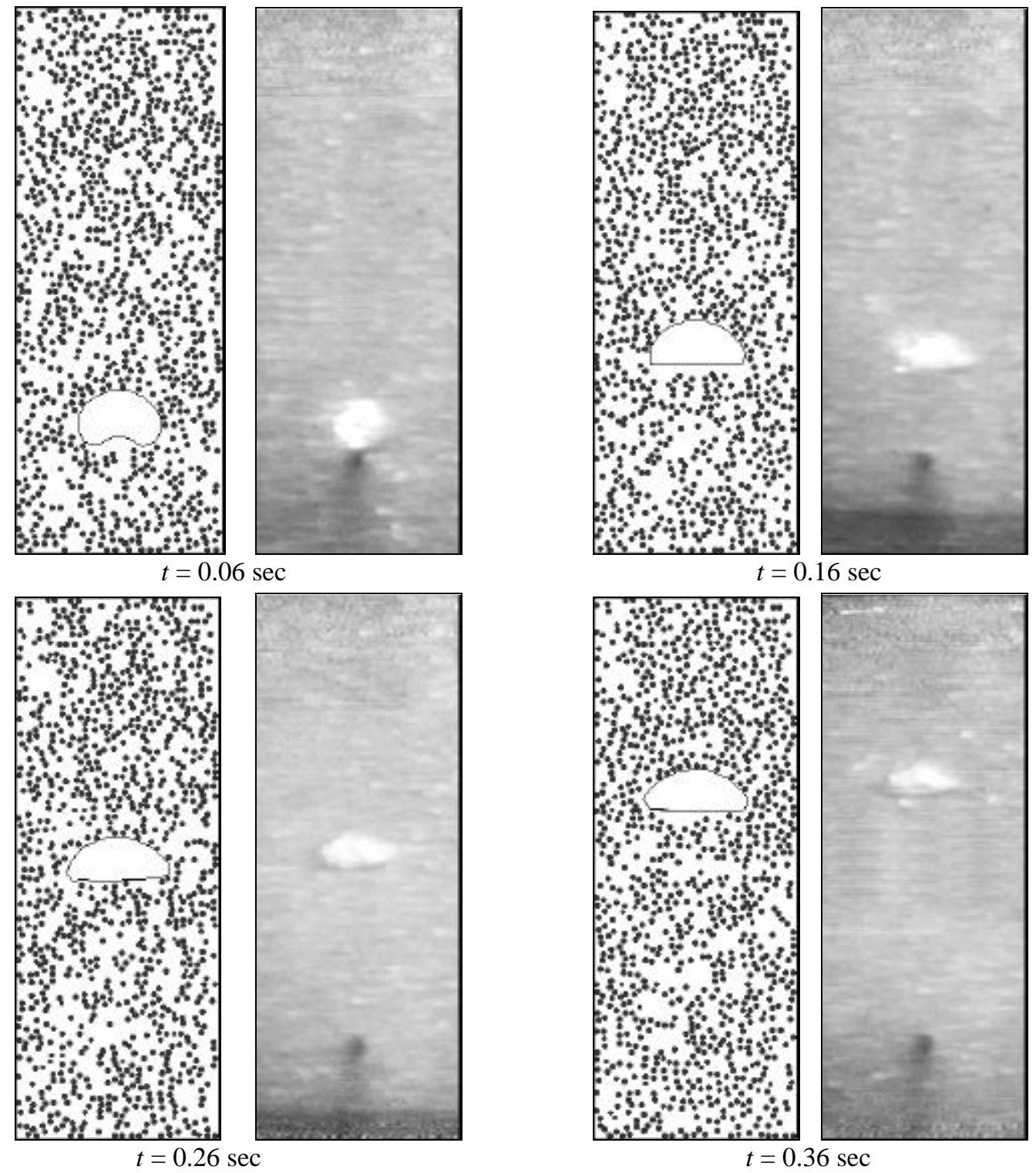

Figure 6.6. $\quad$ Simulation and Experimental Results of a Bubble Rising in a Liquid-Solid Fluidized Bed 

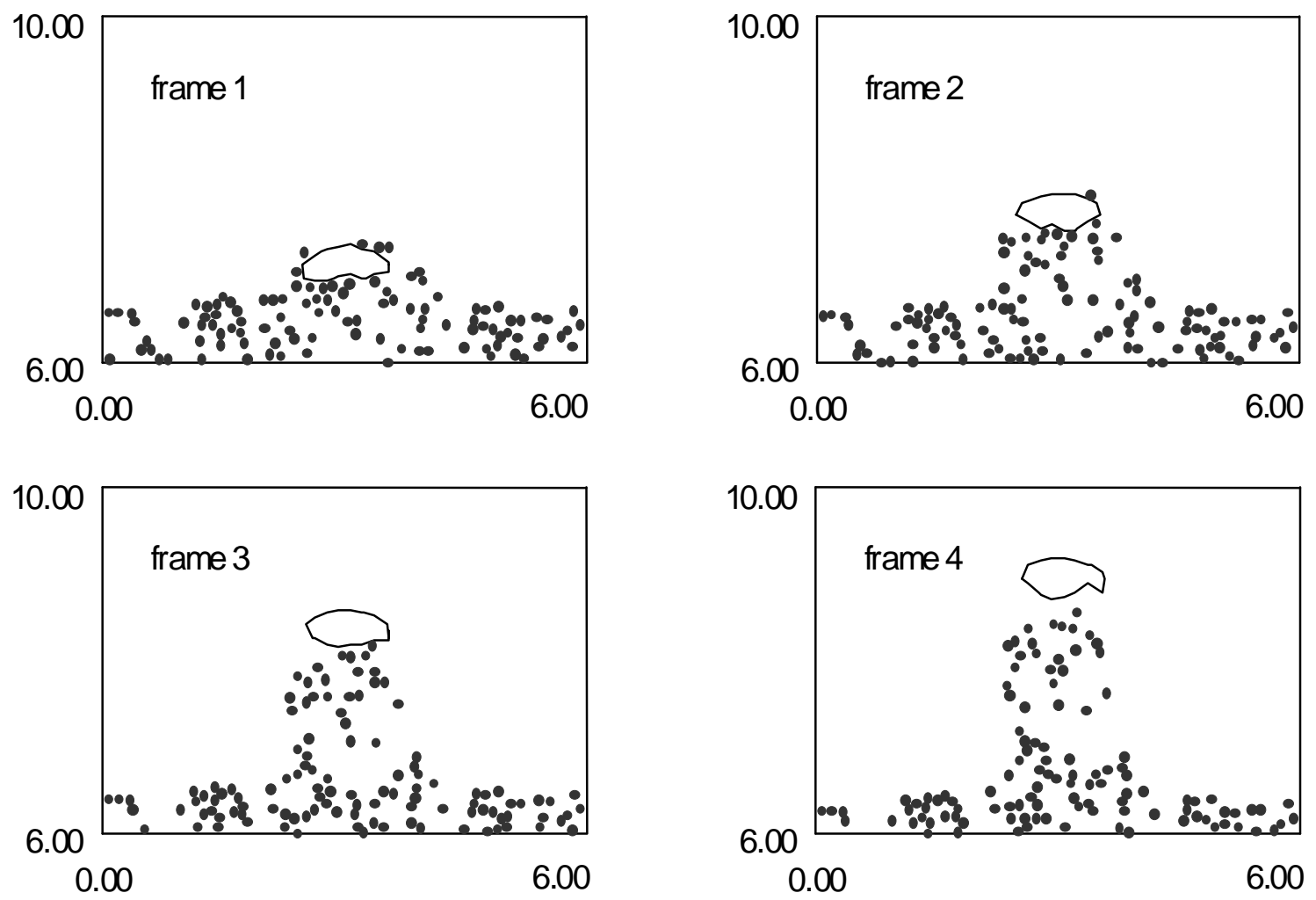

Figure 6.7. Simulation of a Bubble Emerging From a Liquid-Solid Fluidized Bed

\subsection{References}

Allen, M.P. and D.J. Tildesley, 1987. Computer Simulation of Liquids. Clarendon Press, Oxford.

Anderson, T.B. and R. Jackson, 1967. A fluid mechanical description of fluidized beds. I\&EC Fundam. 6, 527-539.

Bhaga, D. and M.E. Weber, 1981. Bubbles in viscous liquids: shapes, wakes, and velocities. J. Fluid Mech., 105, 61.

Brackbill, J.U., D.B. Kothe, and C. Zemach, 1992. A continuum method for modeling surface tension. J. of Comp. Phys. 100, 335-354.

Chen, R.C. and Fan, L.-S., 1992. Particle Image Velocimetry for characterizing the flow structure in three-dimensional gas-liquid-solid fluidized beds. Chem. Eng. Sci., 47, 3615.

Chen, Y.-M. and L.-S. Fan, 1989. Bubble breakage due to particle collision in a liquid medium. Chem. Eng. Sci., 44, 2762-2767. 
Clift, R., J.R. Grace, and M.E. Weber, 1978. Bubbles, Drops, and Particles. Academic Press, New York.

Fan, L.-S., and K. Tsuchiya, 1990. Bubble Wake Dynamics in Liquids and Liquid-Solid Suspensions, Butterworth-Heinemann, Stoneham, MA.

Hirt, C.W. and B.D. Nichols, 1981. Volume of fluid (VOF) method for the dynamics of free boundaries. J. Comp. Phys., 39, 201-225.

Maneri, C.C., 1995. New look at wave analogy for prediction of bubble terminal velocities. AIChE J., 41, 481.

Mendelson, H.D., 1967. The prediction of bubble terminal velocities from wave theory. AIChE J., 13, 250 .

Miyahara, T.K., K. Tsuchiya, and L.-S. Fan, 1989. Mechanism of particle entrainment in a gas-liquid-solid fluidized bed. AIChE J., 35, 1195-1198.

Rowe, P.N. and G.A. Henwood, 1961. Drag forces in a hydraulic model of a fluidized bed - Part I. Trans. Instn. Chem. Engrs., 39, 43-54.

Tomiyama, A., I. Kataoka, and T. Sakaguchi, 1995. Drag coefficients of bubbles (First report, Drag coefficients of a single bubble in a stagnant liquid). Nippon Kikai Gakkai Ronbunshu B Hen, 61(587), 2357.

Tsuchiya, K., G.-H. Song, W.-T. Tang, and L.-S. Fan, 1992. Particle drift induced by a bubble in a liquid-solid fluidized bed with low-density particles. AIChE J., 38, 1847-1851.

Wen, C.Y. and Y.H. Yu, 1966. A generalized method for predicting the minimum fluidization velocity. AIChE J., 12, 610-612.

Zhang, J., L.-S. Fan, C. Zhu, R. Pfeffer, and D. Qi,, 1998. Analytical modeling of collinear collision of elastic spheres moving at low Reynolds numbers in viscous fluids. PTF Topical Conference at AIChE Annual Meeting, Nov. 15-20, Miami Beach, FL. 


\section{DYNAMIC SIMULATION OF BUBBLY FLOW IN BUBBLE COLUMN BY EULERIAN/EULERIAN METHOD}

\subsection{Introduction}

Bubble column reactors are widely used in chemical industry due to their excellent heat transfer characteristics and simple construction. The fundamental properties of the hydrodynamics in bubble columns, which are essential for scale-up and design, are still not fully understood at the current stage mainly due to the complicated nature of multiphase flow. Based on the experimental evidence, for example, the works by Tzeng et al. (1993) and Devanathan et al. (1995), it is clear that, beside the time-averaged quantities, the transient behavior of flow is required to provide further information on the fluid dynamics and transport parameters in such reactors. For instance, to study the mixing process and develop the axial dispersion models which are commonly adopted in the design and evaluation of bubble column performance, one needs the time-dependent velocity field for calculating the convection and turbulent dispersion of the passive scalar in the liquid and/or gas phase. Currently, the experimental techniques for multiphase flow include the computer-automated radioactive particle tracking (CARPT) and the particle image velocimetry (PIV). The former is a Lagrangian measurement. Devanathan et al. (1990) and Yang et al. (1995) have employed this technique to investigate the liquid velocity field in cylindrical bubble columns. The PIV system, originally developed for the velocity measurement in single-phase flow, has recently been used for multiphase systems at low volume fraction of the dispersed phase. One of the advantages of PIV technique is its capability of providing the instantaneous velocity field in the place of investigation. This type of measurement for the gas-liquid flow in bubble columns has been realized by Chen and Fan (1992) and Chen et al. (1994). With the rapid development of computational fluid dynamics (CFD) and increase in the computing power, the numerical simulation of multiphase flow is being recognized as a potential primary tool for the investigating and thus improving the performance of various multiphase contactors like bubble columns.

Two-phase bubbly flow is commonly defined as a flow pattern in which the gas phase is distributed within a liquid continuum in discretized bubbles much smaller than the characteristic dimension of the container (e.g. column diameter). Numerical simulation of twophase flows either treats the system in an averaged sense as interpenetrating continuum, or explicitly moves bubbles due to the fluid-imposed and body forces---in some cases feeding back the effect of the bubbles on the carrier-fluid motion through various means. The first type of approach is called Eulerian/Eulerian method, while the second is usually referred to as Eulerian/Lagrangian method. In the first the two-fluid model is developed to describe the motion for each of the two phases in Eulerian frame of reference. In the second approach, while the continuous phase is still described in the Eulerian representation, the dispersed phase is instead treated as it is, i.e. the discrete bubbles, and each bubble is tracked by solving its equation of motion through the continuous phase. Both approaches have their own advantages and disadvantages and are commonly employed in fundamental research and engineering applications. A review on the state of art of the modeling of gas-liquid flow in bubble columns and loop reactors can be found in Sokolichin and Eigenberger (1994). 
During the last several years the dynamic simulation of gas-liquid flow in bubble column reactors has drawn a considerable attention of the investigators in chemical reaction engineering community. Webb et al.(1992) and Lapin and Lubbert (1994) studied the gasliquid flows in two-dimensional bubble columns. They used a single-fluid model in which the two-phase flow is regarded as a quasi-single-phase flow with variable density. Bubbles are considered to individually rise in this fluid flow, thus, leading to a dynamical change of its density which finally results in a convective flow of the bubble/liquid two-phase system. The gas phase motion is calculated by either solving the bubble distribution density function or individually tracking the bubbles or bubble clusters in a Lagrangian frame. The liquid circulation pattern in the columns of low aspect ratio has been observed in their simulation. Using an Eulerian/Eulerian model, Sokolichin and Eigenberger (1994) presented a laminar, dynamic two-dimensional simulation of gas-liquid bubble flow in a flat and uniformly aerated bubble column. In their study, no turbulence model was used in solving the velocity filed of the liquid phase and the drag force was calculated with a constant drag coefficient which leads to a mean bubble slip velocity of about $20 \mathrm{~cm} / \mathrm{s}$ for air bubbles of $1-10 \mathrm{~mm}$ mean diameter in water. Their results are further compared with the laboratory observations and data in a flat bubble column and a loop reactor by Becker et al. (1994). The experimental techniques employed were Laser Doppler anemometer (LDA) for liquid velocity, a fiber optical probe for bubble velocity and an electroconductivity probe for gas holdup. The experiments and simulations were conducted in a partially aerated bubble column operating at low superficial gas velocities (about $0.3-0.6 \mathrm{~cm} / \mathrm{s}$ ) and in an airlift loop reactor. They showed good qualitative agreement of the numerical results and data for both the steady state and the transient behavior. By simply increasing the liquid's molecular viscosity by a factor of 100 to account for the influence of turbulence viscosity, they found a good quantitative agreement as well. However the quantitative comparisons presented in the paper are very limited (In fact, only a number of gas holdup profiles in an airlift loop reactor are shown.).

In a series of papers, Delnoij et al. $(1997 ; 1997 \mathrm{a} ; 1997 \mathrm{~b})$ numerically investigated the gasliquid flow in two-dimensional bubble columns by Eulerian/Lagrangian methods. The motion of gas phase was solved by applying either discrete bubble model or volume of fluid (VOF) model. Unlike the work by Lapin and Lubbert (1994) where the coupling between the gas and liquid phase was achieved through the effective density of the mixture and no momentum exchange was incorporated, Delnoij et al. (1997) coupled the two phases by adding a source term, which includes all the forces imposed on the liquid surrounding the bubbles, into the volume-averaged Navier-Stokes equation of the liquid phase. The gas phase was described by the equations of motion for each individual bubble. In order to prevent bubble-bubble overlap during the simulation, a collision model was also developed for direct bubble-bubble interaction. Again, no turbulence model was used in the simulation. The authors compared the results, mostly in a qualitative way, with the experiment on a partially aerated quasi-twodimensional bubble column by Becker et al. (1994) and studied the effect of column aspect ratio on the flow structure.

In the field of numerical study on multiphase hydrodynamics of bubble columns, considerable efforts have been devoted to the Eulerian/Lagrangian type of simulations, mostly twodimensional, in the recent year due to, may be, the tremendous growth in computing power. It is also well understood that the Eulerian/Lagrangian method is more suitable for fundamental 
investigations since it allows for a direct consideration of various effects related to bubblebubble and bubble-liquid interaction. Instead, for practical applications, in which the high gas superficial velocity results in high gas holdup and turbulence or even churn turbulence, Eulerian/Eulerian method is usually preferred. However, several challenging issues regarding to the modeling of averaged equations and closure relations, which have been the active research topic in the field of multiphase flow for many years, still exist. One of these issues is how to model the inter-phase momentum exchange which is related to the problem of calculating the force acting on the bubble and taking into account the effect of multi-bubble (or finite value of gas holdup) on these forces. Another unresolved issue is the modeling of turbulence in two-phase flow.

It is noted that the previous studies compared the numerical predictions with the experimental measurement mostly in a qualitative way while only a few, if any, quantitative comparisons were made. Therefore, although the qualitative comparisons are satisfactory in general, limited conclusion regarding the validation and reliability of the numerical prediction can be drawn from these studies. This is, perhaps, partially due to the difficulties in getting the reliable measurement for multiphase system. Recently Lin et al. (1996) and Mudde et al. (1997) presented their experimental studies on two-dimensional bubble column at low gas volume fraction by using the PIV technique. They provided the detailed measurement of liquid velocity and turbulence intensities for the columns of different sizes and under different operating conditions. They also studied the characteristics of the macroscopic flow structures, i.e. the central meandering plume and the companion vortical regions, by measuring their frequency, wave-length and moving speed. This information provided a better understanding of gas-liquid flow in a 2-D bubble column and generated a database for the further investigations.

In the present study we present an Eulerian/Eulerian dynamic simulation of two dimensional gas-liquid bubble column. The ensemble-averaged equations are used to solve the velocity and volume fraction field for both phases. A model of bubble-induced turbulent viscosity is incorporated into the momentum equations for the liquid phase. The effect of the gas volume fraction on the inter-phase momentum exchange term is also included. The numerical predictions of macroscopic structures and mean properties are compared with the experimental data provided by Mudde et al. (1997) and Lin et al. (1996). By these comparisons, we verify that the dynamic simulation of Eulerian/Eulerian type is able to capture the flow structure in a 2-D bubble column and provide a satisfactory quantitative results for the cases which are within the bubbly flow regime.

\subsection{Ensemble Averaged Equations for Two-Phase Flow}

Many attempts have been made to derive the averaged equations for disperse two-phase flows (see, e.g. Drew 1983; Wallis 1991: Zhang and Prosperetti (1997). Either the volume averaging or the ensemble averaging technique can be used to derive the equations of so-called 'twofluid' form widely used in engineering. In the recent ensemble averaging approach present by Zhang and Prosperetti (1997), the exact Navier-Stokes equations for the continuous phase is averaged by using the phase ensemble averaging method (i.e. averaged over all the configurations such that at time $t$ the position $\mathbf{x}$ is occupied by the continuous phase). For the 
disperse phase a method of 'particle' ensemble average is introduced, in which global particle attributes (e.g. the velocity of the center of mass) are averaged directly. In other word the averaged equations for the dispersed phase are obtained by directly ensemble averaging the equation of motion of particles where each particle is treated as a single identity.

For the incompressible liquid and gas the continuity equations for each phase are written as

$\frac{\partial \varepsilon_{c}}{\partial t}+\nabla \cdot\left(\varepsilon_{c} \mathbf{u}_{c}\right)=0$

and

$\frac{\partial \varepsilon_{d}}{\partial t}+\nabla \cdot\left(\varepsilon_{d} \mathbf{u}_{d}\right)=0$

The momentum equation for the liquid phase is written as

$\rho_{c} \varepsilon_{c}\left(\frac{\partial \mathbf{u}_{c}}{\partial t}+\mathbf{u}_{c} . \nabla \mathbf{u}_{c}\right)=\rho_{c} \varepsilon_{c} \mathbf{g}-\varepsilon_{c} \nabla p-\left(\mathbf{M}_{d}+\mathbf{M}_{v m}\right)+\nabla .\left(\varepsilon_{c} \boldsymbol{\sigma}_{c}\right)+\nabla .\left(\varepsilon_{c} \boldsymbol{\sigma}_{c}^{b}\right)$

where the stress tensor, $\sigma_{c}$, is related with the velocity field by

$\sigma_{c}=\mu_{c}^{*}\left(\nabla \mathbf{u}_{c}+\nabla \mathbf{u}_{c}^{\top}\right)$

where $\mu_{c}^{*}$ is the well-known effective viscosity of a dilute suspension of particles (see, e.g. Batchelor (1967))

$\frac{\mu_{c}^{*}}{\mu_{c}}=1+\frac{5}{2} \varepsilon_{d}+O\left(\varepsilon_{d}\right)$

The inter-phase momentum exchange terms describing the drag force $M_{d}$, and added mass force $M_{v m}$, are defined as

$\mathbf{M}_{d}=\frac{6 \varepsilon_{c} \varepsilon_{d}}{\pi d_{p}^{3}} \mathbf{F}_{d}$

and

$\mathbf{M}_{v m}=\frac{1}{2} \varepsilon_{c} \varepsilon_{d} C_{v m}\left(\frac{D \mathbf{u}_{c}}{D t}-\frac{D \mathbf{u}_{d}}{D t}\right)$

where $\frac{D}{D t}$ represents the substantial derivative. 
As derived by Zhang and Prosperetti (1997), the momentum equation for the gas phase can be obtained by ensemble averaging the equation of motion of a spherical bubble moving through fluid, which gives,

$$
\rho_{d} \varepsilon_{d}\left(\frac{\partial \mathbf{u}_{d}}{\partial t}+\mathbf{u}_{d} \cdot \nabla \mathbf{u}_{d}\right)=\rho_{d} \varepsilon_{d} \mathbf{g}-\varepsilon_{d} \nabla p+\left(\mathbf{M}_{d}+\mathbf{M}_{v m}\right)
$$

Since each bubble moves as a whole object, the rotational and the internal motion of the bubbles are neglected. As a result a term related to the shear stress of the gas phase vanishes. Equation 8 explicitly indicates that the bubbles respond to the continuous-phase pressure, rather than to some dispersed-phase pressure. This feature is in agreement with physical intuition. The dispersed-phase press, i.e. the pressure inside the bubble, cannot affect the motion of the bubbles directly, but only indirectly through its relation with the continuousphase pressure resulting from dynamic boundary conditions at the bubble surface. In the present situation there is no need to solve the momentum equation for the gas, but only to state that the gas pressure is spatially uniform inside each bubble.

For quantitative analysis, it is necessary not only to derive the correct form of these equations but also to obtain reliable estimates of the various averaged quantities, such as the viscous drag and added mass coefficients that appear in the averaged equations. As is well known, the drag force acting on a spherical object moving at $u_{d}$ through a fluid with velocity $u_{c}$ can be expressed as

$$
\mathbf{F}_{d}=\frac{1}{8} \rho_{c} \pi d_{p}^{2} C_{D}\left|\mathbf{u}_{c}-\mathbf{u}_{d}\right|\left(\mathbf{u}_{c}-\mathbf{u}_{d}\right)
$$

The drag coefficient $C_{D}$ depends on the flow regime and the liquid properties. As a classical problem, the drag coefficient of a gas bubble in liquid has been extensively studied through the years. A widely used reference is Clift et al. (1978). Here we used the recent results by Tsuchiya et al. (1997). The drag coefficient of a bubble in a sufficiently contaminated system can be expressed by

$$
C_{D}=\max \left[\frac{24}{\operatorname{Re}}\left(1+0.15 \operatorname{Re}^{0.687}\right), f \frac{8}{3} \frac{E o}{E o+4}\right]
$$

where the Eotvos number, Eo, is defined as

$$
E o \equiv g \rho_{c} d_{p}^{2} / \tau
$$

The bubble Reynolds number, $R e$, is based on the bubble diameter $d_{p}$, liquid viscosity $v$ and the relative velocity between phase, i.e. the slip velocity. The effect of gas content on $C_{D}$ is 
taken into account by modifying the liquid viscosity as $\mu_{m}=\mu_{c} / \varepsilon_{d}$ in the evaluation of the Reynolds number and by multiplying the second part of Equation 10 with

$$
f=\left\{\frac{1+17.67 \varepsilon_{c}^{9 / 7}}{18.67 \varepsilon_{c}^{3 / 2}}\right\}^{2}
$$

Additional resistance due to a bubble is caused by the relative acceleration of the bubble in the liquid. This is the added mass force given by Equation 7. Generally the virtual mass coefficient $C_{v m}$ is a function of the volume fraction of the gas phase $\varepsilon_{d}$ with a leading term of value 1. It may also depend on the mass density ratio of the continuous and dispersed phase, i.e. $\rho_{d} / \rho_{c}$, as recently proposed by Zhang and Prosperetti (1996). Zuber's (1964) well-known results for $C_{v m}$ is

$$
C_{v m}=\frac{1+2 \varepsilon_{d}}{1-\varepsilon_{d}}
$$

Wijingaaden (1976) and Biesheuvel and Spoelstra (1989) calculated $C_{v m}$ in the dilute limit and found respectively,

$$
\begin{aligned}
& C_{v m}=1+2.76 \varepsilon_{d}+O\left(\varepsilon_{d}^{2}\right) \\
& C_{v m}=1+3.32 \varepsilon_{d}+O\left(\varepsilon_{d}^{2}\right)
\end{aligned}
$$

The difference is due to the different velocity distribution assumed in the calculation. For the cases being considered in the present study where the gas holdup does not exceed $10 \%$, we expect the above first-order expressions to provide a give good approximation, and we choose to use the one by Biesheuvel and Spoelstra (1989), given by Equation (14b).

To predict momentum transfer in bubbly flow it is important to elucidate turbulence of the continuous liquid phase, which may result due to the presence of bubbles. As proposed by Sato and Sekoguchi (1975) the turbulent stress in the liquid phase of bubbly flow can be subdivided into two components, one due to the inherent, i.e. shear-induced, turbulence which is independent of the relative motion of bubbles and the other due to the additional turbulence caused by bubble agitation, i.e. bubble-induced turbulence. Experimental evidence (see Lance and Bataille (1991), and Theofanous and Sullivian (1984)) show that for low holdup bubbly flow the two parts are only weakly coupled so that a linear superposition can be applied. As mentioned before we only model the bubble-induced turbulence.

$$
\boldsymbol{\sigma}_{c}^{b}=-\rho_{c} \overline{\mathbf{u}_{c}{ }^{\prime} \mathbf{u}_{c}{ }^{\prime}}
$$

By applying the eddy viscosity model, Sato et al. (1981) suggested, 
$\boldsymbol{\sigma}_{c}^{b}=\rho_{c} \boldsymbol{v}_{b}^{t}\left(\nabla \mathbf{u}_{c}+\nabla \mathbf{u}_{\mathbf{c}}^{\mathbf{\top}}\right)$

and

$\boldsymbol{v}_{b}^{t}=k_{b} \varepsilon_{d} d_{p}\left|\mathbf{u}_{c}-\mathbf{u}_{d}\right|$

where $k_{b}$ is an empirical constant which usually takes a value of 1.2. This model for the bubble-induced enhancement in viscosity is based on the concept of mixing length, where the bubble radius is taken to be the bubble-induced turbulence length scale.

\subsection{Numerical Details}

A package, CFDLIB, developed by the Los Alamos National Laboratory (see Kashiwa and Rauenzahn (1994)), is used for the simulations presented in this work. CFDLIB is a hydrocode library for multiphase flow simulations. It uses a cell-centered finite-volume method applied to the time-dependent equations of conservation. Some parts of the code related to the interphase momentum exchange and turbulence calculations are modified according to the models discussed in the last section.

Figure 7.1 shows the mesh system used for the $15-\mathrm{cm}$ wide column. The grid sizes in horizontal and vertical direction are $0.5 \mathrm{~cm}$ and $0.8 \mathrm{~cm}$, respectively. In order to obtain a better comparison with experimental data, we set the conditions for our simulations as close to those in Mudde et al.'s (1997) experiment as possible. The flow conditions and column sizes for each run are listed in Table 7-1. The gas injectors are numerically realized by setting openings at the bottom of the column which allow only the gas phase passing through at a velocity that, together with gas holdup, matches the value of the gas superficial velocity used in the experiment. Since the size of gas injectors used in the experiments (e.g., $0.16 \mathrm{~cm}$ in diameter) is too small to be resolved in the numerical simulation with the currently employed mesh, the openings are about 2 to 4 times larger than the real injectors. It should be noted that in Figure 7.1 the grid is finer at the locations of the gas injectors.

All simulations start from a static initial condition where the main body of the column is filled with water and the top part only with gas. The simulations are then performed until a quasisteady state is reached. The time-averaged quantities are then calculated as defined in the following expressions,

$$
\begin{aligned}
& \bar{u}(x)=\frac{1}{N_{t}} \sum_{n=1}^{n=N_{t}} u_{n}(x) ; \quad \bar{v}(x)=\frac{1}{N_{t}} \sum_{n=1}^{n=N_{t}} v_{n}(x) \\
& \overline{u^{\prime} u^{\prime}}=\frac{1}{N_{t}} \sum_{n=1}^{n=N_{t}} u_{n}^{2}-\bar{u}^{2} ; \quad \overline{v^{\prime} v^{\prime}}=\frac{1}{N_{t}} \sum_{n=1}^{n=N_{t}} v_{n}^{2}-\bar{v}^{2} ; \quad \overline{u^{\prime} v^{\prime}}=\frac{1}{N_{t}} \sum_{n=1}^{n=N_{t}}\left(u_{n}-\bar{u}\right)\left(v_{n}-\bar{v}\right)
\end{aligned}
$$




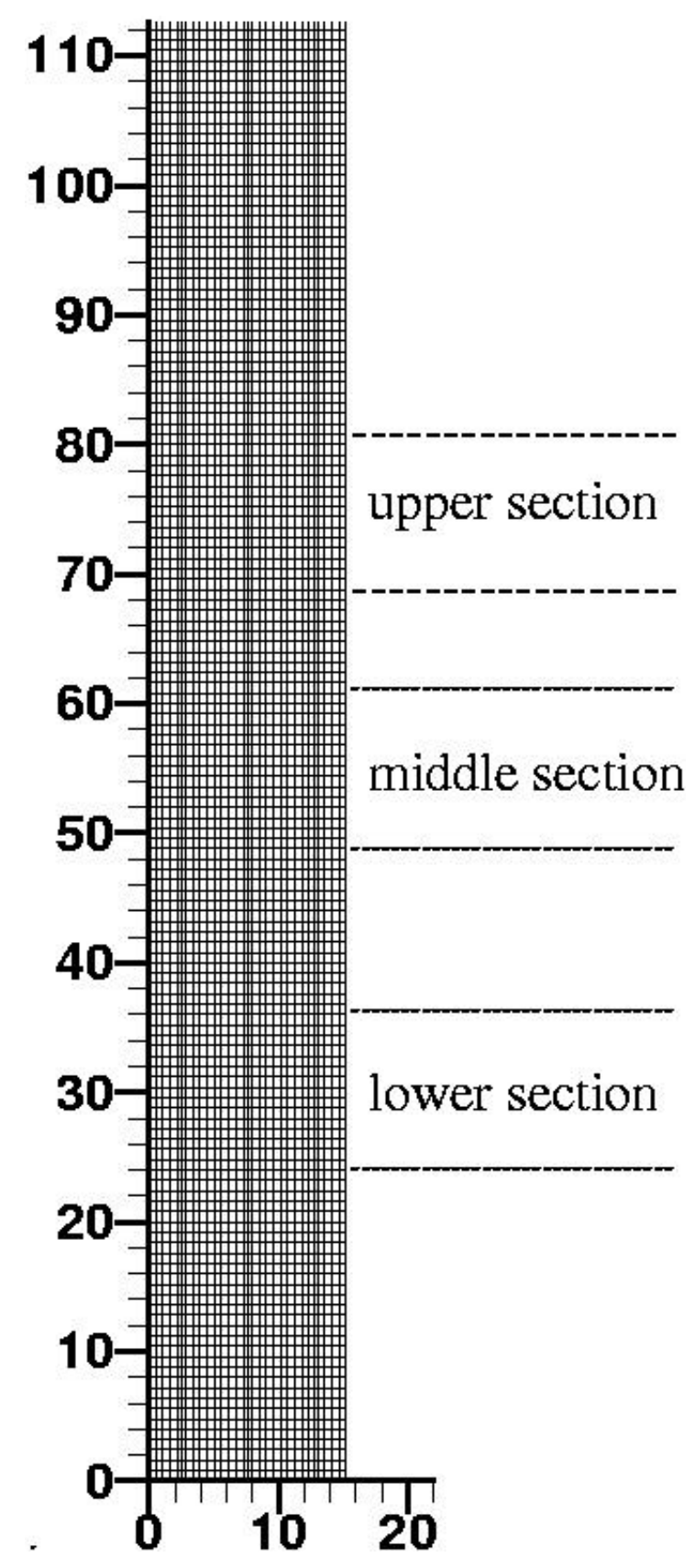

Figure 7.1: Computational Mesh Used For the Simulations of 2-D Column With Discrete Gas Injectors 
where $u_{n}(x)$ and $v_{n}(x)$ are the horizontal and vertical components of the liquid velocity at time step $\mathrm{n}$. In all simulations the velocity and gas holdup fields are sampled every 0.1 second. To ensure the convergence of the averaged quantities the averaging processes are performed for 50 to 100 seconds which corresponds to a period of time during which about 10 to 20 meandering waves pass through a point at the center-line of the column. The spatial averaging is then performed along the vertical direction within the lower, middle and upper sections as shown in Figure 7.1, respectively. The locations and sizes of these three sections are exactly as same as in Mudde et al.'s (1997) experiment.

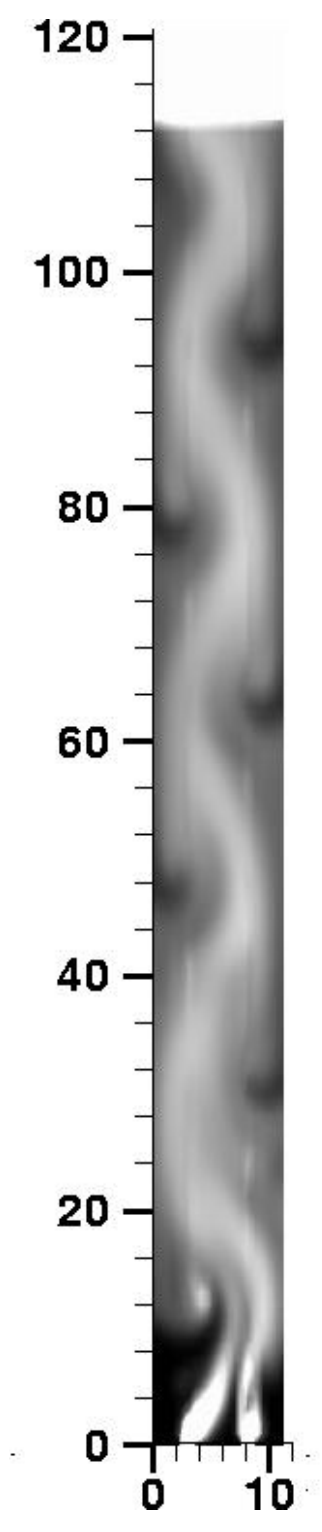

(1)

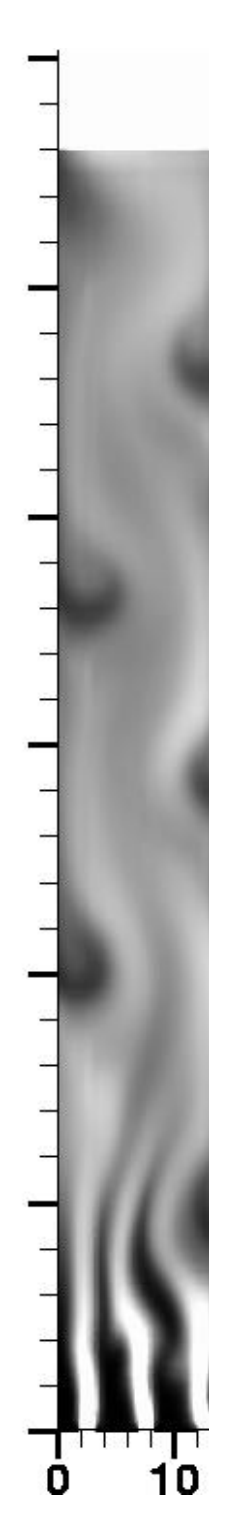

(2)

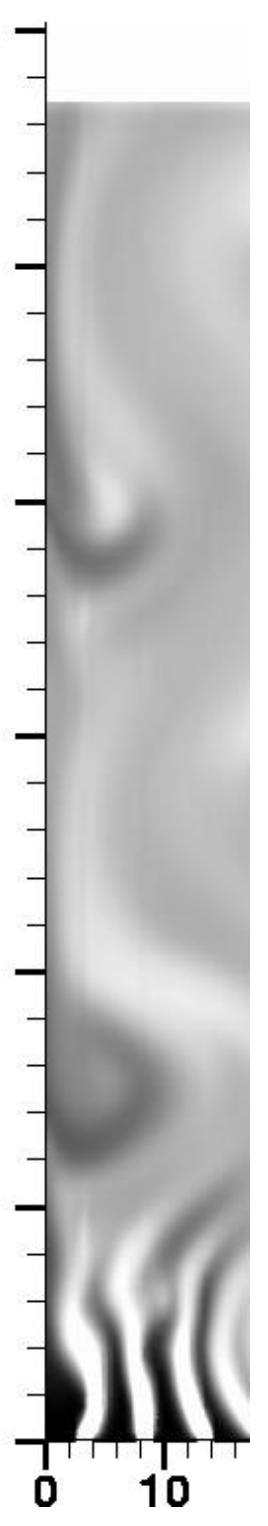

(3)

Figure 7.2: $\quad$ Instantaneous Contour Plots for Gas Holdup in (1) 11.2-cm Wide Column at $\mathrm{U}_{\text {sup }}=1(\mathrm{Cm} / \mathrm{s})$; (2) 15.2-cm Wide Column at $\mathrm{U}_{\text {sup }}=1(\mathrm{Cm} / \mathrm{s})$; and (3) 32-cm Wide Column at $\mathrm{U}_{\text {sup }}=1.2(\mathrm{Cm} / \mathrm{s})$ 


\subsection{Large Structures in Bubble Columns}

Chen et al. (1989) have observed, in their visual study of two-dimensional bubble columns, that when the liquid depth exceeds the column width, i.e. when column's aspect ratio exceeds one, a central wave-like plume accompanied with two staggered rows of vortices which resemble the Karman vortex street appear. Tzeng et al. (1993) and Lin et al. (1996) further classified the large flow structures in 2-D bubble columns into four distinct flow regions, namely central plume region, fast bubble flow region, vortical flow region and descending flow region. Figure 7.2 shows a snapshot of the instantaneous flow patterns in three columns with different width at the same gas superficial velocity. The central plume with relatively high gas holdup and the vortices adjacent to the side walls are clearly visible. To study the dynamic properties of these large structures we have performed the animations of the flow field by using the velocities and gas holdup generated from the simulations. These animations show that the bubbles injected at the bottom cluster together to move upward in a wavy-like manner while the vortex pairs drift down. The multiple vortex cells that are continually generated in the vortical flow region become confined by the wave motion of central plume. The behavior of these vortices is dynamic in nature, and the formation of these vortices at each side wall appears to be independent of each other. The entire vortex region is swinging laterally back and forth corresponding to the wave motion of the central plume. The structures can be further examined by enlarging the view of a portion of the column. Figure 7.3 shows a snapshot of the liquid velocity vector, liquid vorticity contour and gas holdup contour in a 15$\mathrm{cm}$ wide column. The velocity vectors and vorticity contours clearly indicate the counterrotating vortex pairs along the side walls.

The dynamic behavior of the large structures is characterized by the wave length and frequency of the meandering central plume. It is expected that these quantities vary with column size and gas (superficial) velocity. Lin et al. (1996) have conducted an extensive and detailed experimental investigation, in 2-D columns, on this topic. They have found that, in the same column, the frequency increases with gas velocity while the wave length decreases. At the same gas superficial velocity the frequency decreases as the size (width) of the column increases. The wave-length is proportional to the column size. When the wave length and frequency are multiplied to give the vortex descending velocity, they found that it is basically a function of the gas velocity only. They also found that the size of these vortices is independent of gas velocity and varies with column size only. Figure 7.4 shows the flow pattern in a $15-\mathrm{cm}$ wide column under three different gas velocities. As consistent with the experimental observation, wave length decreases and the overall gas holdup increases as the gas velocity increases. If one performs the spatial fourier transform of the liquid velocity, the wave-length should corresponds to the peak of the power spectra. In figure 7.5 the spatial power spectra of the horizontal component of the liquid velocity field for the three cases depicted in Figure 7.4 are shown. They are calculated by conducting the fourier transform of u along the center line of the column. This value is then averaged over time. As the gas velocity increases, the shifting of the peak towards a larger waver number clearly indicates the shortening of the wave-length. The calculated primary wave-length is $50 \mathrm{~cm}, 32 \mathrm{~cm}$ and $25 \mathrm{~cm}$ for $U_{\text {sup }}=0.25,1.0$ and $2.0(\mathrm{~cm} / \mathrm{s})$, respectively. The measured values, as shown in the figure 8 of Lin et al.'s paper, are $25 \mathrm{~cm}$ and $21 \mathrm{~cm}$ for $U_{\text {sup }}=1.0$ and $2.0 \mathrm{~cm}$, respectively. The difference between the calculated and measured values may partially be due to the fact that the wave length is measured visually in the experiment while it is obtained from the power spectra 
in the simulation. Also the smallest variation in wave-length is limited by the spatial resolution used in the simulation. Nonetheless the general trend is certainly captured and consistent with the experimental observations. To study the flow structure in time/frequency domain, we record a time series of liquid velocity at the central point of the column. Figure 7.6 shows such a sample. The meandering motion of the central plume is clearly demonstrated, that is, periods of positive and negative horizontal velocity component alternate. It is interesting to note that while the horizontal motion is dominated by a primary frequency, the vertical motion seems to contain several comparable frequencies. Figure 7.7 shows the temporal power spectra of both $\mathrm{u}$ and $\mathrm{v}$ at the center line of the column. They are calculated by averaging the spectra at all points on the center line within a middle section of a $15-\mathrm{cm}$ wide column. For the horizontal xcomponent of liquid velocity the second peak is two order of magnitude smaller than the first one. On the other hand, two comparable peaks appear in the spectra of the vertical ycomponent of liquid velocity. It should be noted that turbulence is present in both components. Again the calculated primary period is 6.4 seconds while the measured one is 4.4 second as shown in figure 9 of Lin et al. (1996). 


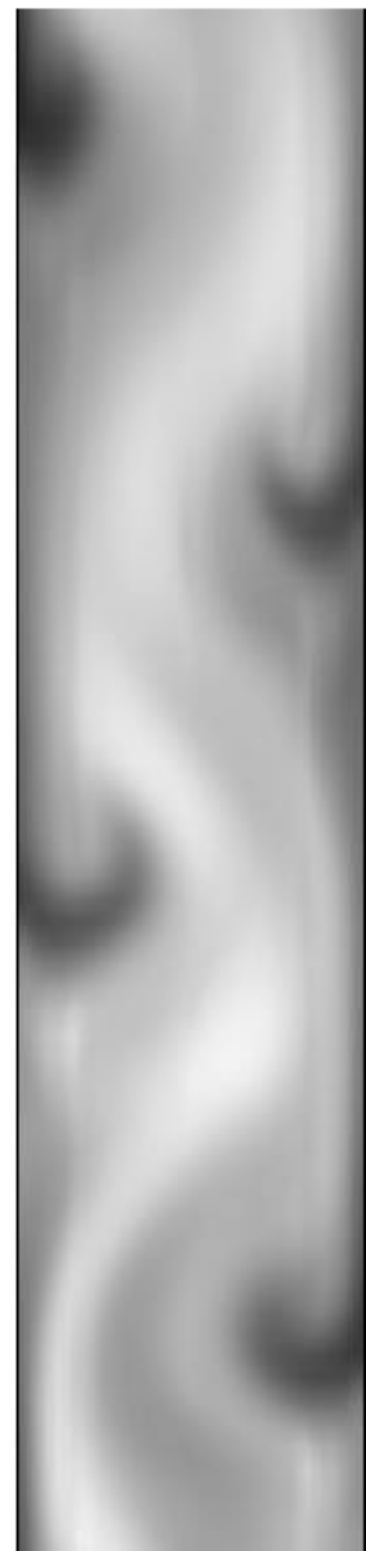

(1)

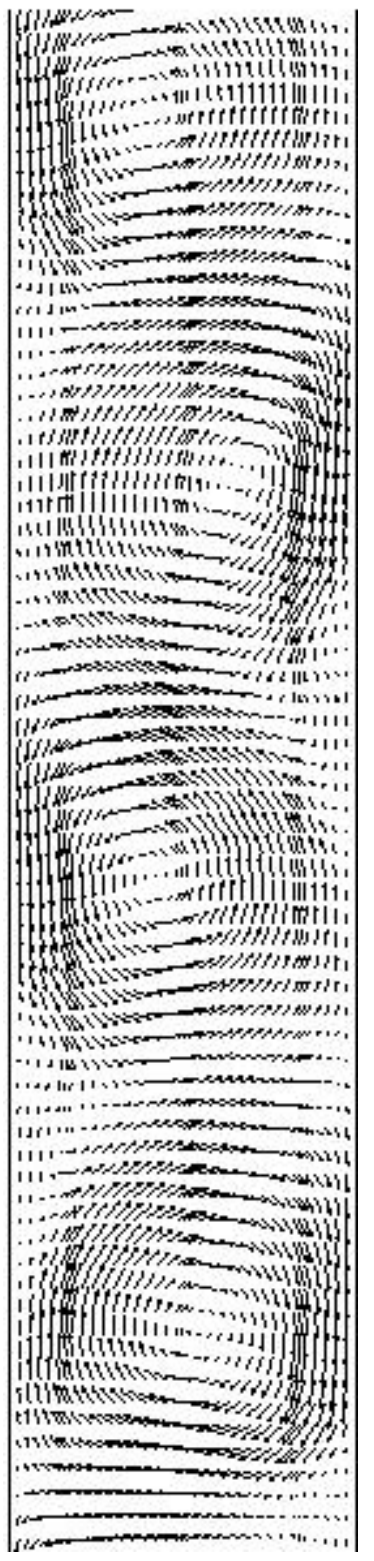

(2)

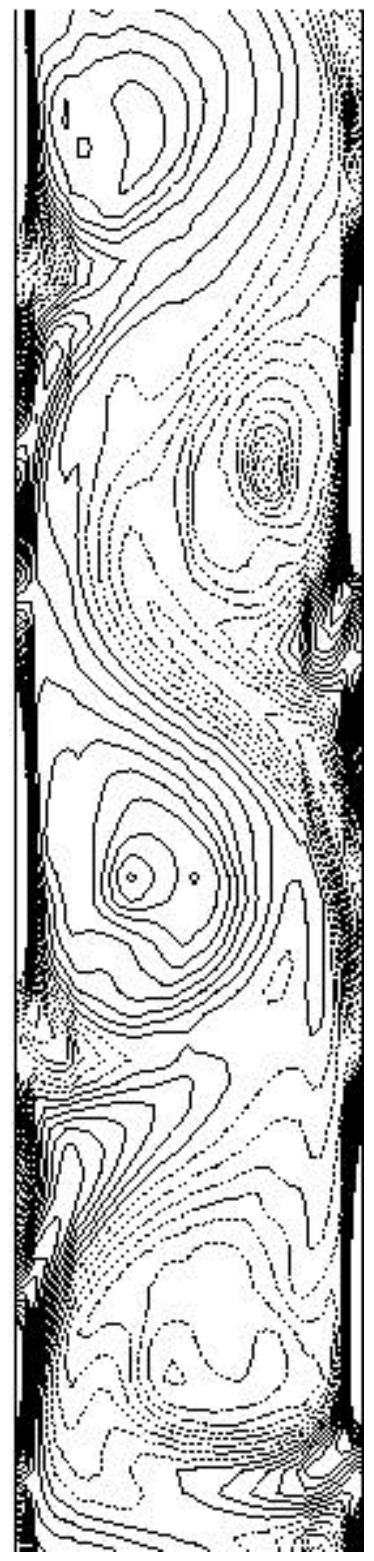

(3)

Figure 7.3: The Instantaneous Flow Field in a 15-cm Wide Column at $U_{\text {sup }}=1(\mathrm{~cm} / \mathrm{s})$. (1) Gas Holdup Contour; (2) Liquid Velocity Vector; (3) Liquid Vorticity Contour 


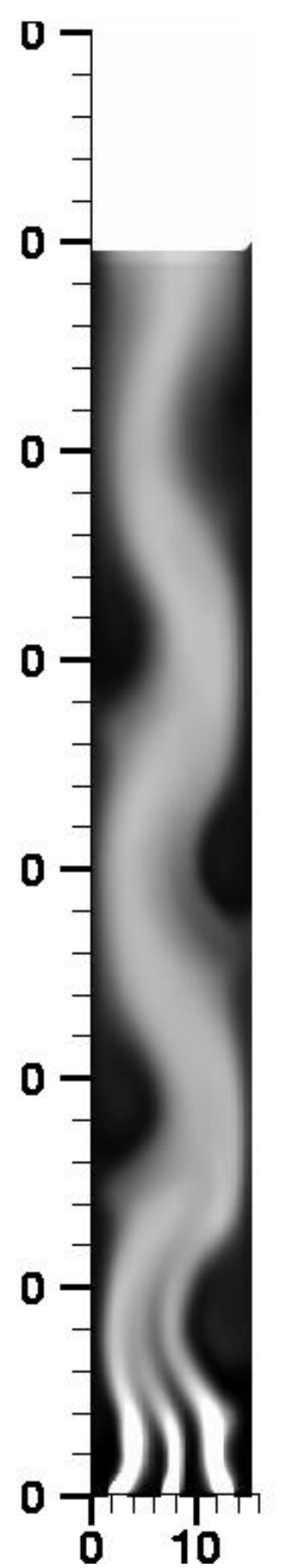

(1)

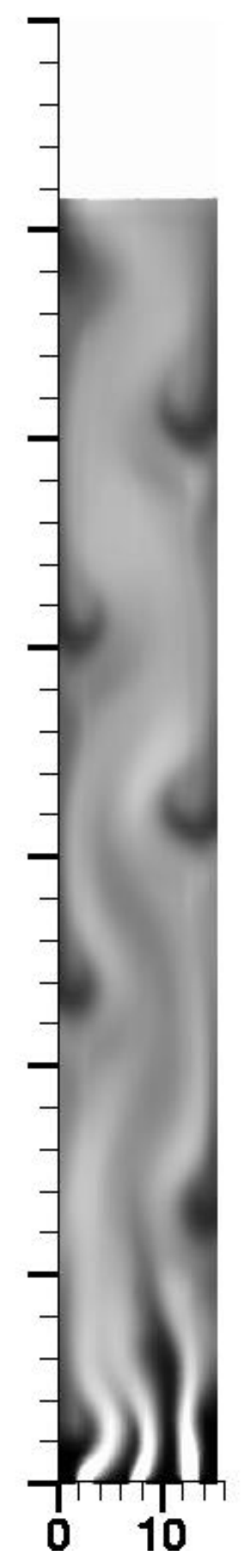

(2)

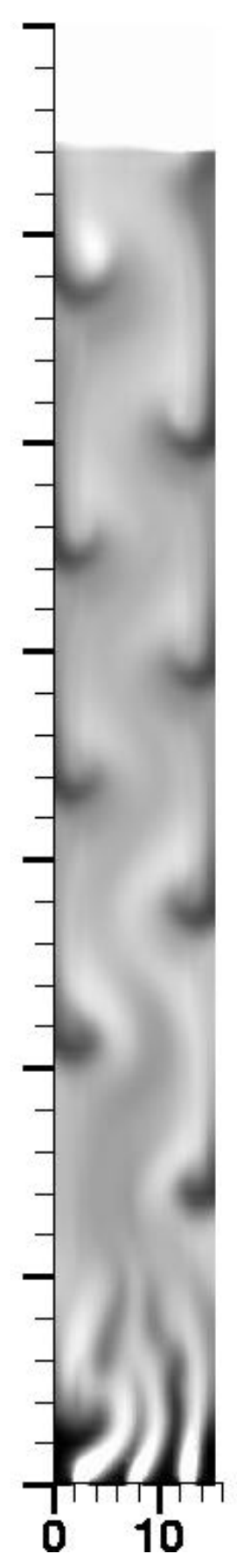

(3)

Figure 7.4: Instantaneous Gas Holdup Contours in a $15.2 \mathrm{~cm}$ Wide Column Operated at (1) $U_{\text {sup }}=0.25 \mathrm{~cm} / \mathrm{s}$; (2) $U_{\text {sup }}=1 \mathrm{~cm} / \mathrm{s}$; (3) $U_{\text {sup }}=2 \mathrm{~cm} / \mathrm{s}$ 


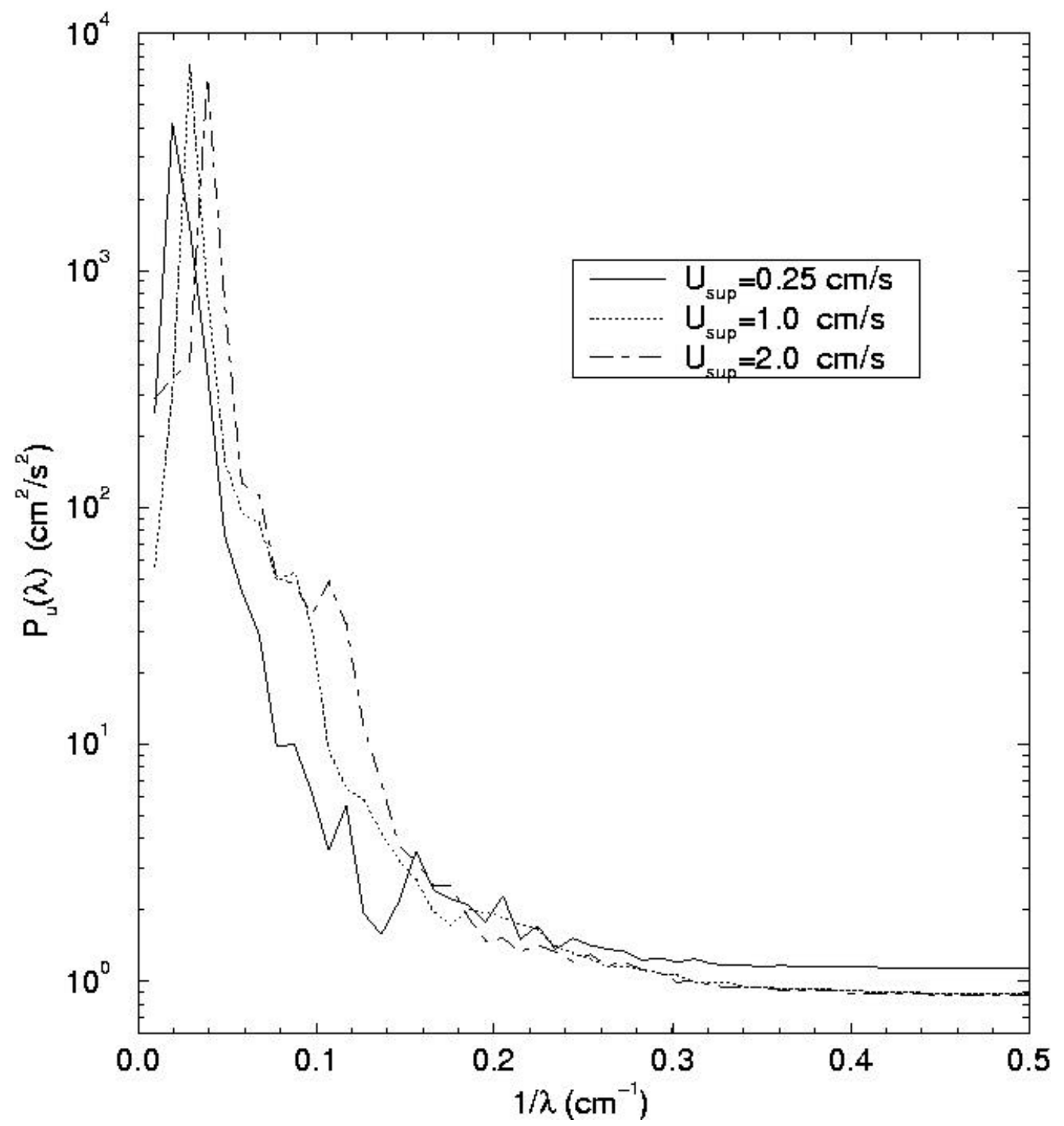

Figure 7.5: Spatial Power Spectra of $\mathrm{u}$ in a 15-cm Wide Column at Three Different Superficial Gas Velocities 

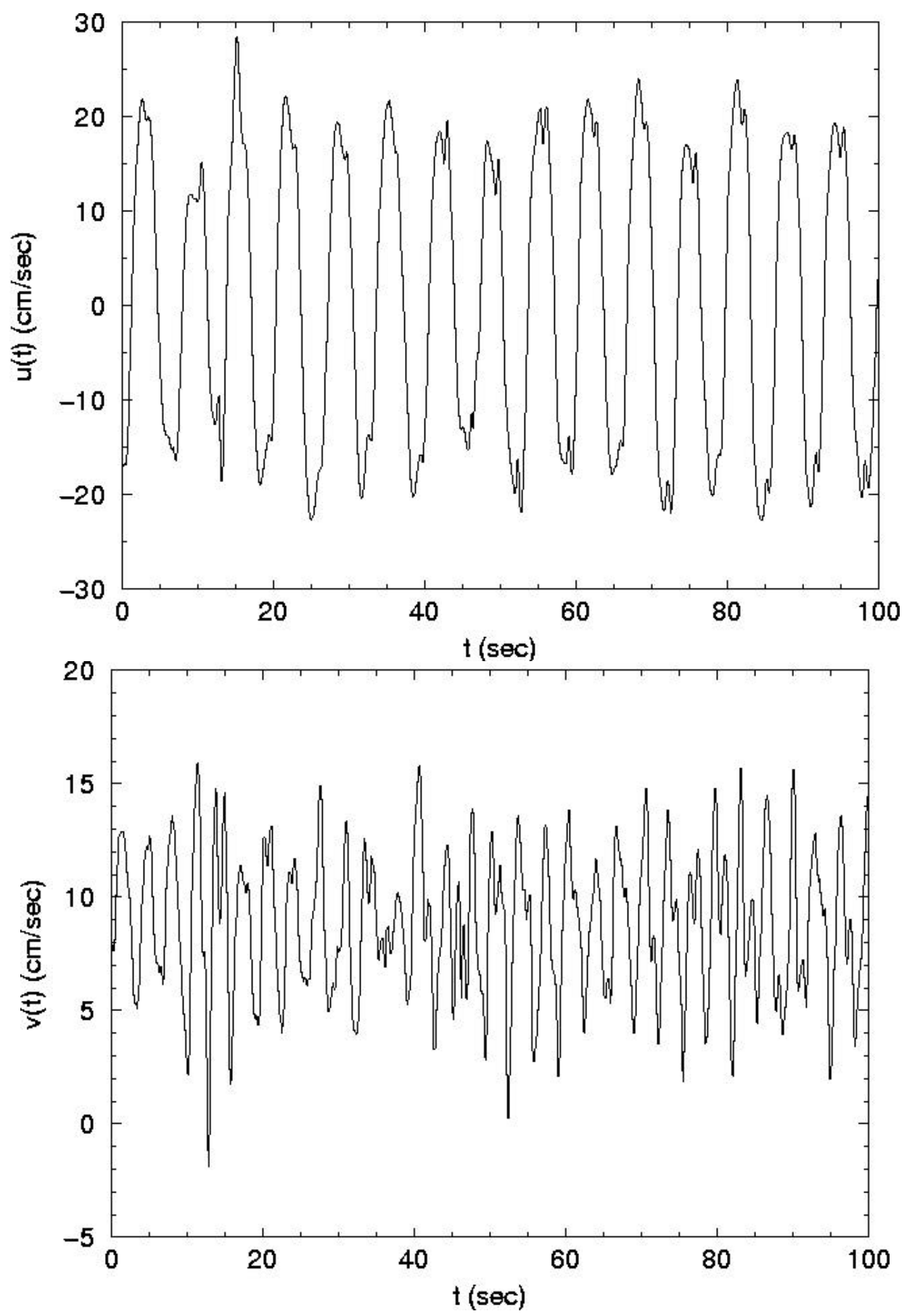

Figure 7.6: Time Series of the Liquid Velocity at the Central Point of 15-cm Wide Column at $\mathrm{U}_{\text {sup }}=1 \mathrm{~cm} / \mathrm{s}$ 

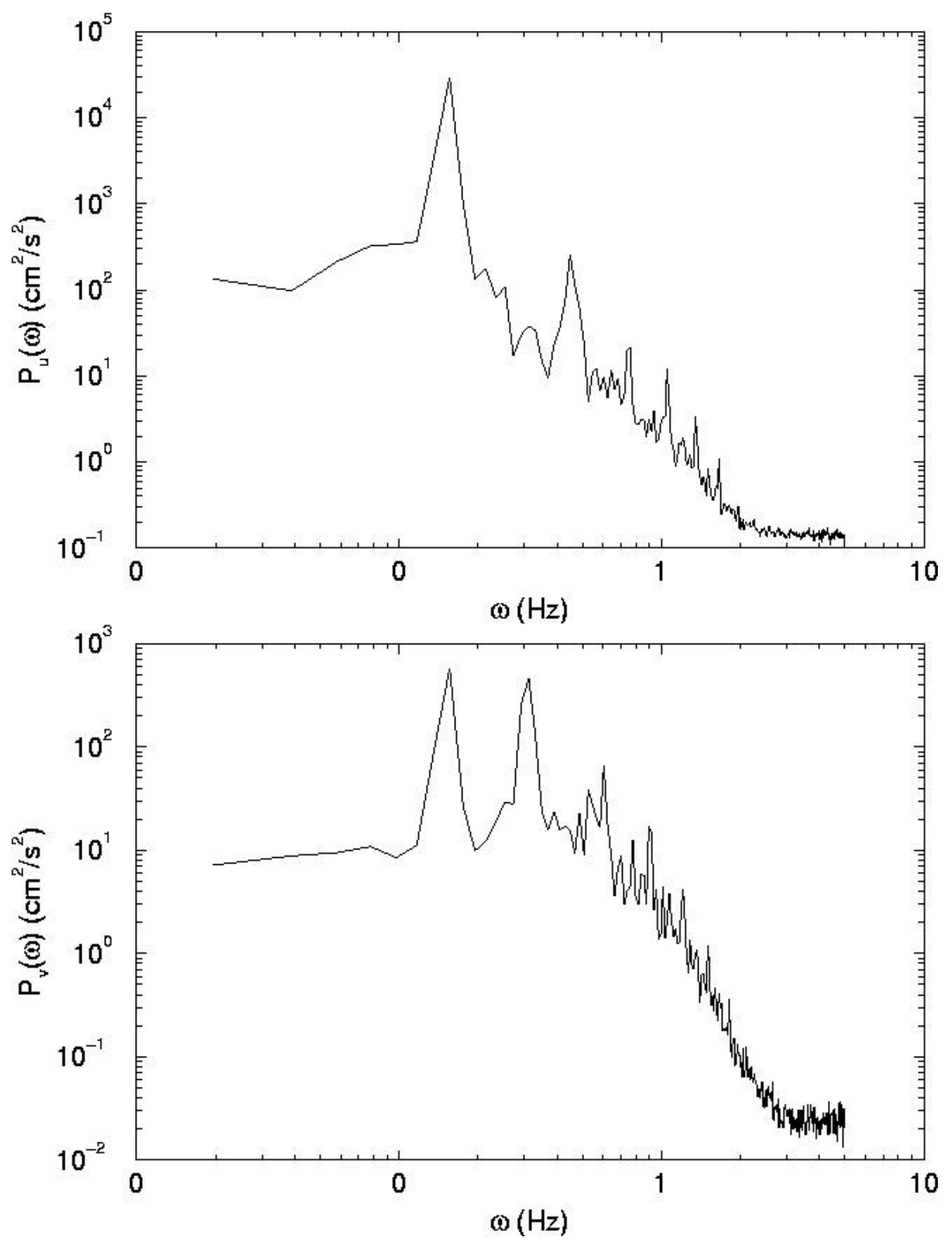

Figure 7.7: Temporal Power Spectra of the Liquid Velocity in a 15-cm Wide Column at $\mathrm{U}_{\text {sup }}=1 \mathrm{~cm} / \mathrm{s}$ 


\subsection{Effects of Gas Distributors}

One of the major topic in studies of bubble column reactors for engineering application is the effect of the different type of gas spargers on the characteristics of the flow field and in turn on the mass/heat transfer properties. Numerical simulation may provide useful information in this regard. We have made a group of simulations of a $15-\mathrm{cm}$ wide column. Each simulation uses a different gas distributor, which are: one, three, and nine injectors and a uniform distributor. The injectors are set symmetric about the center-line of the column. By uniform distributor we mean that the gas velocity at the inlet, i.e. at the bottom of the column, is uniformly set to a constant value. Among these four cases, the one with the uniform distributor is considered as the closest to realty. In most bubble column reactors, the gas is introduced into the column through many tiny holes on a perforated plate. The size of these holes is usually of the order of $0.1 \mathrm{~mm}$ or even smaller, and the holes are distributed uniformly on the plate. A perforated plate, whose open area is of the order of $0.1 \%$, would have hundreds holes. Obviously it is impossible to resolve the holes in a numerical simulation. Thus an uniform distribution of gas velocity and volume fraction at the bottom would be a reasonable boundary condition for simulations.

In the cases simulated, while the gas is injected in different ways into the column, the superficial velocity is kept the same for all the runs. Figure 7.8 shows the flow pattern for these four cases. One notes that the overall gas holdup for each case is the same. This indicates that the overall gas holdup is not affected by the gas distributors and depends on the gas superficial velocity $U_{\text {sup }}$ only. Some differences are observed in the large structures in each case. The wave length of the meandering structure in the columns with three and nine injectors are smaller than those with one and uniform distributors. The mean liquid velocity and gas holdup profiles for the middle section of the column are shown in Figure 7.9. One can see that the differences between all the cases are quite small. It is thus confirmed that changes in gas distributors induce only limited effects on the mean properties and flow structures.

\subsection{Quantitative Comparison With Experiments}

To further verify our numerical results, we conducted quantitative comparisons of the mean velocity, turbulence intensities and shear stress profiles with the experimental data provided by Mudde et al. (1997). Figure 7.10 shows the vertical and horizontal mean velocity profiles of a 11-cm wide column at $1 \mathrm{~cm} / \mathrm{s}$ gas superficial velocity. The compared profiles are for the middle section of the column where the mean flow is usually assumed one dimensional. The numerical prediction of the mean horizontal velocity, $\bar{u}$, is essentially zero as expected. The experimental data, however, is non-zero and exhibits an inward flow. As pointed out by Mudde et al. (1997), this is attributed to a systematic error due to the difficulty in tracking the particles in the fast-moving bubble stream and other biased measurements. The comparison of the mean vertical velocity profile is quite good except in the near-wall region. One reason for this discrepancy is that the boundary layer is too thin to be resolved in our current simulations. 

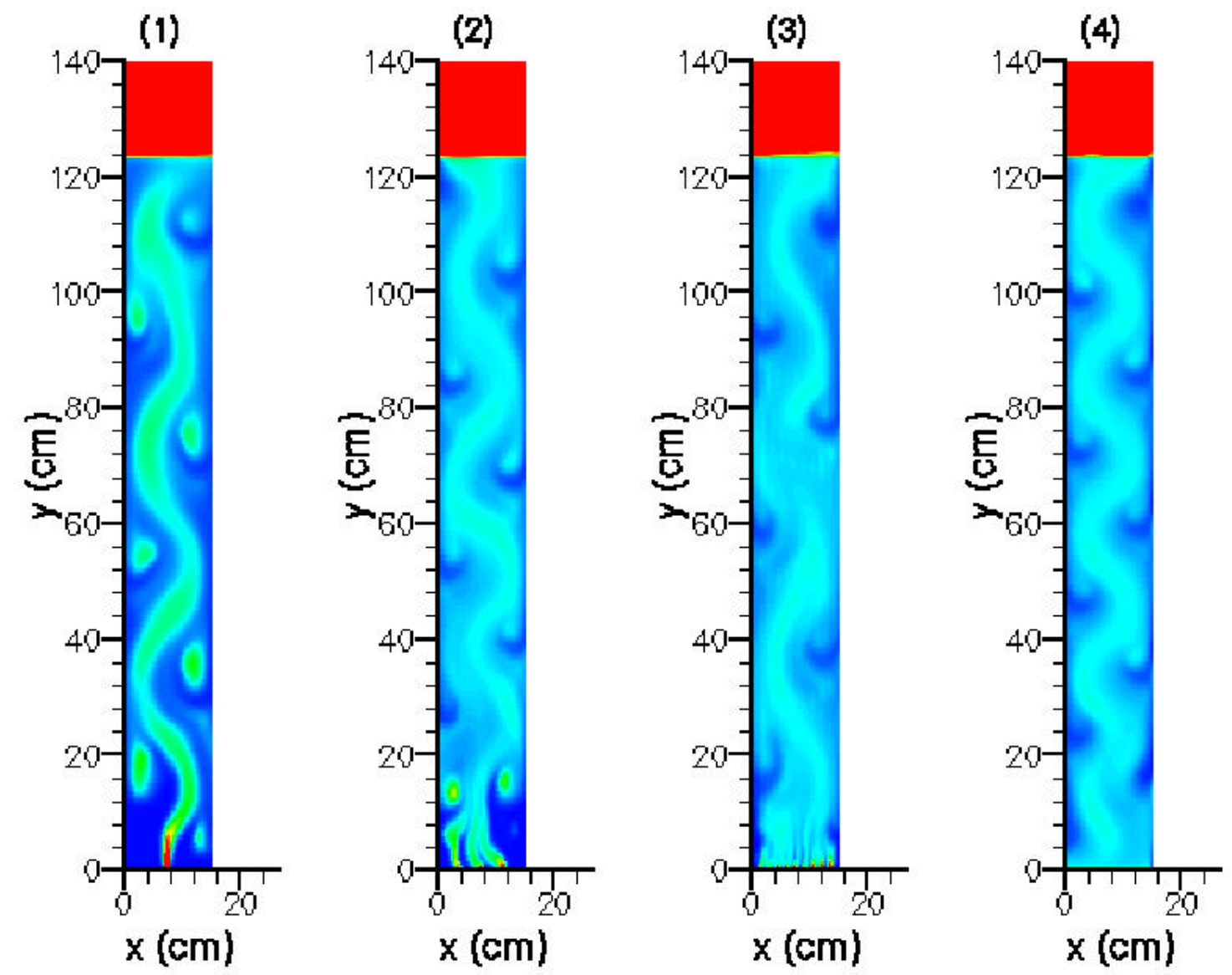

Figure 7.8: The Instantaneous Gas Holdup Contour of $15-\mathrm{cm}$ Wide Column at $\mathrm{U}_{\text {sup }}=1 \mathrm{~cm} / \mathrm{s}$ with: (1) 1-jet; (2) 3-jet; (3) 9-jet; (4) Uniform Gas Distributor 

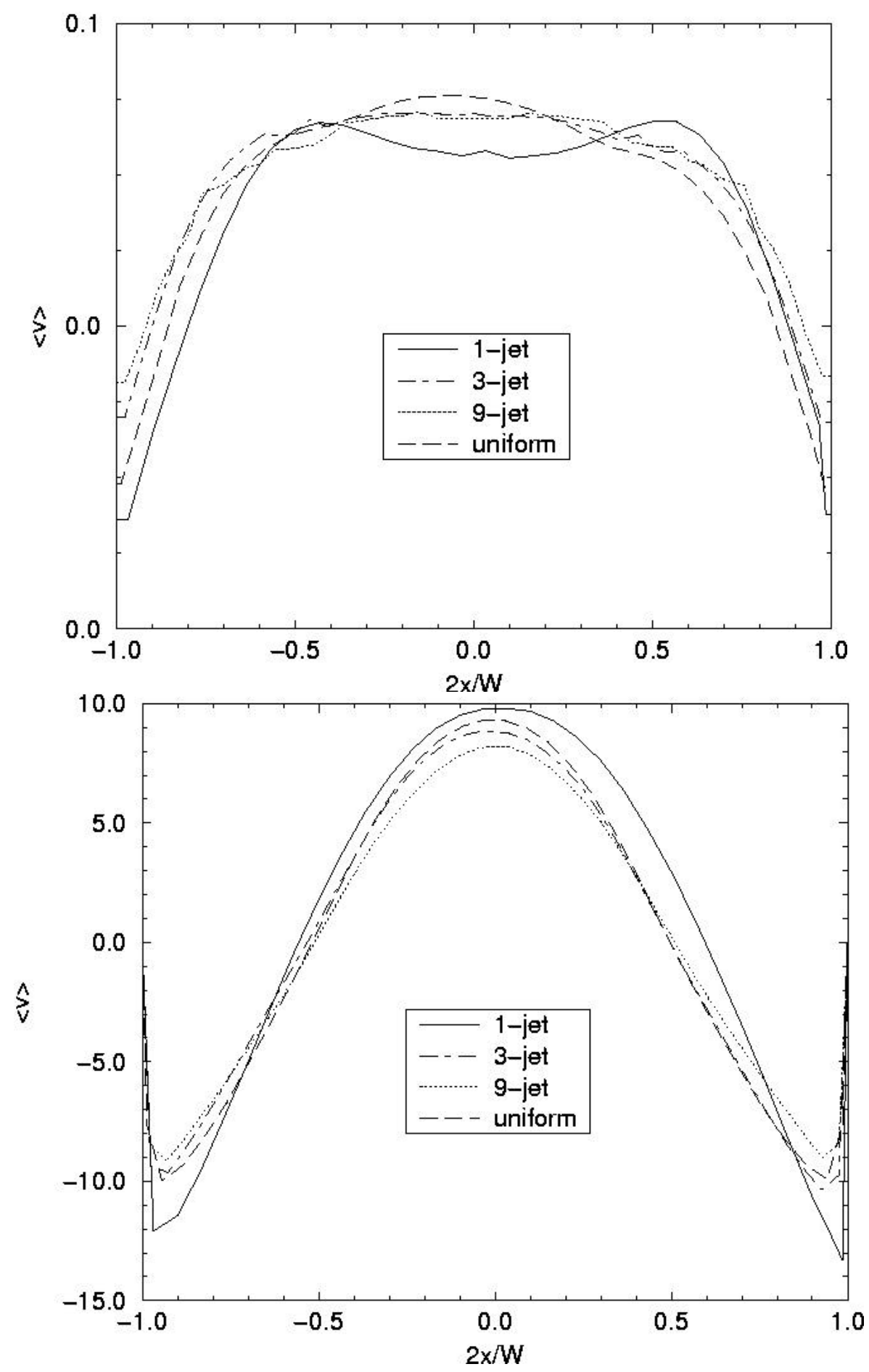

Figure 7.9: Time Averaged Profiles Gas Holdup and the Vertical Velocity of the Liquid Phase at the Middle Section of $15-\mathrm{cm}$ Wide Column at $\mathrm{U}_{\text {sup }}=1(\mathrm{~cm} / \mathrm{s})$, With Different Gas Distributors 


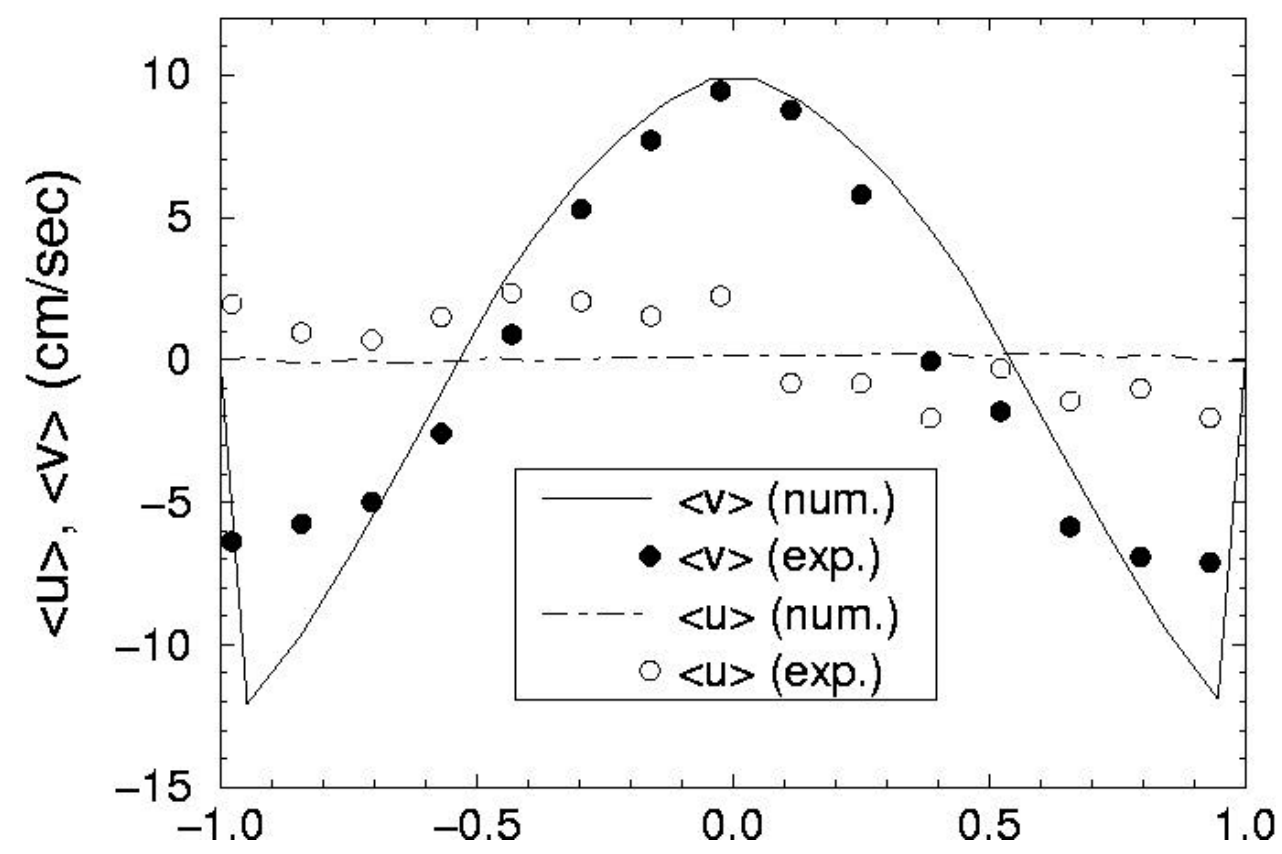

Figure 7.10: Time-Averaged Velocity Profiles for the Middle Section of 11-cm Wide Column at $\mathrm{U}_{\text {sup }}=1(\mathrm{~cm} / \mathrm{s})$

For a 15-cm wide column, the comparisons of the mean vertical liquid velocity are made for the middle, lower and upper sections, as shown in figure 7.11. Again the numerical values match the data quite well particularly in the lower section. The case of a 32-cm wide column is also compared with the experimental data as shown in Figure 7.12. Since the window of PIV measurement covers only half of the column, the data is provided for the left half of the column. Notice that the gas superficial velocity for this case is relatively high, i.e. $U_{\text {sup }}=1.9$ $\mathrm{cm} / \mathrm{s}$. Still the comparison is very satisfactory.

The numerical prediction of turbulence intensities, $\overline{u^{\prime} u^{\prime}}$ and $\overline{v^{\prime} v^{\prime}}$, and Reynolds shear stress, $\overline{u^{\prime} v^{\prime}}$, of $15-\mathrm{cm}$ column under $U_{\text {sup }}=1.0(\mathrm{~cm} / \mathrm{s})$ are compared with data in Figure 7.13. The $\overline{u^{\prime} u^{\prime}}$ reaches a peak at the central portion of the column since $u$ attain its highest magnitude in the center due to the nature of the meandering motion of the central plume. The peaks of $\overline{v^{\prime} v^{\prime}}$ at the near-wall vortical region are consistent with the fact that the flow dynamically changes from upward to downward in such areas. Although the general trend of the numerical values match the data, there are significant differences in the values. It is clear that the simulation under predicts the turbulence related properties. This simply means that our simulations are not able to resolve all the scales of turbulence. However, the good comparisons of mean velocity profile indicate that the effects of turbulence, here mainly the bubble-induced turbulence, are properly modeled and included in the calculation. 

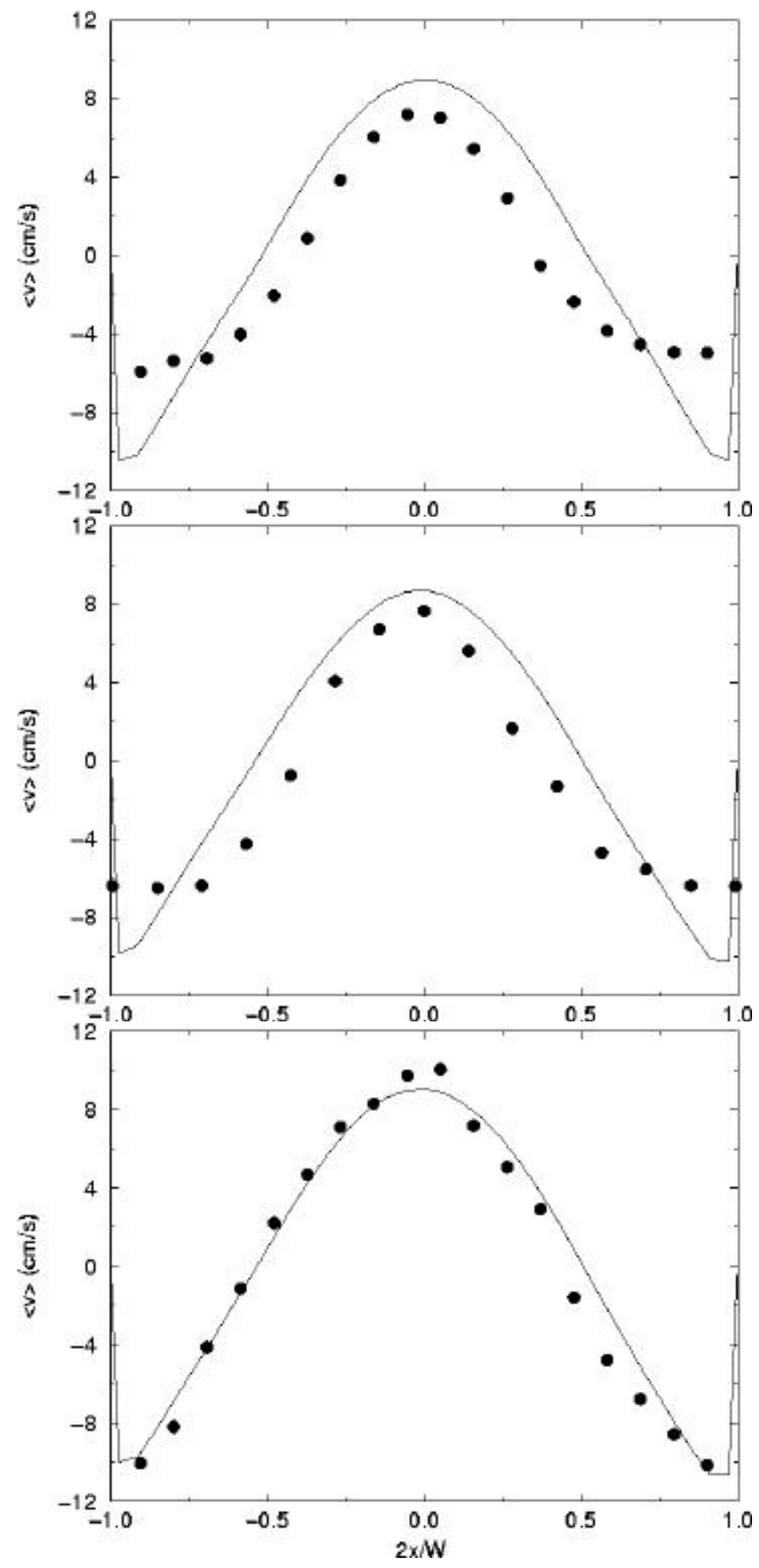

Figure 7.11: Time-Averaged Velocity Profiles for $15-\mathrm{cm}$ Wide Column at $U_{\text {sup }}=1 \mathrm{~cm} / \mathrm{s}$. Top: Upper Section; Middle: Middle Section; Bottom: Lower Section. — and • are the Numerical Prediction and Experimental Data, Respectively 


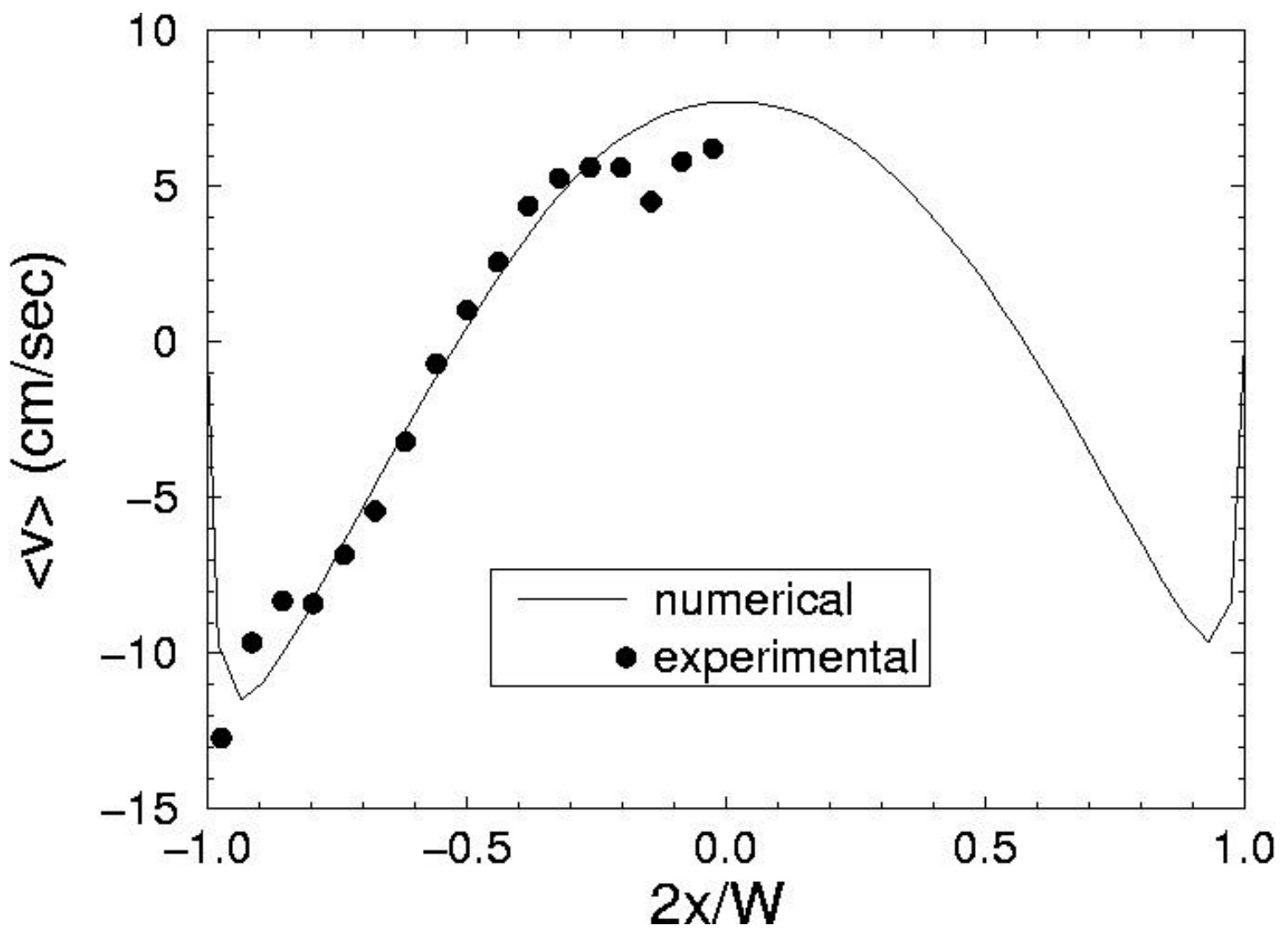

Figure 7.12: Time-Averaged Velocity Profile for the Middle Section of a 32-cm wide Column at $\mathrm{U}_{\text {sup }}=1.9 \mathrm{~cm} / \mathrm{s}$ 

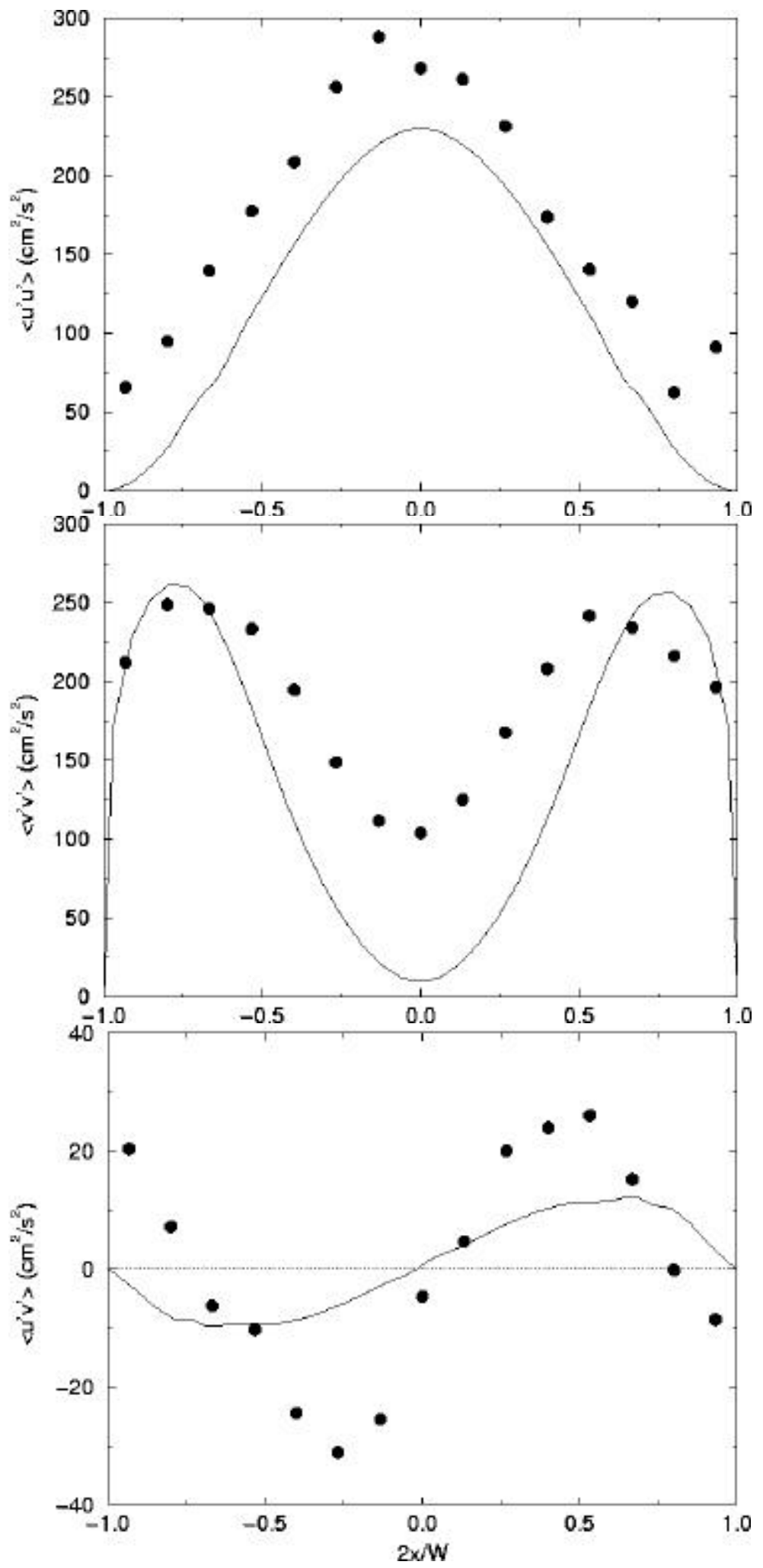

Figure 7.13: Turbulence Intensities and Reynolds Shear Stress in the Middle Section of 15$\mathrm{cm}$ Wide Column Operated at $\mathrm{U}_{\text {sup }}=1 \mathrm{~cm} / \mathrm{s}$. — is the Numerical Values; $\bullet$ is the Experimental Data by Mudde et al. (1997) 


\subsection{Conclusions}

The numerical simulation of gas-liquid flow in two-dimensional bubble columns using Eulerian/Eulerian two-fluid model is able to capture the characteristics of large structures. Quantitative comparisons with the experimental data reveal that, by applying simple models of inter-phase momentum transfer and bubble-induced turbulent viscosity in the liquid phase, the two-fluid simulations provide satisfactory results for the mean flow quantities at least for the case of dispersed bubbly flow, i.e. low gas superficial velocities. To extend the work to higher gas velocity and three-dimensional column, more sophisticated models for the forces on bubbles and turbulence may be needed.

Table 7.1: $\quad$ Column Size and Flow Conditions

\begin{tabular}{|c|c|c|c|c|}
\hline $\begin{array}{c}\text { Column width } \\
(\mathrm{cm})\end{array}$ & $\begin{array}{c}U_{\text {sup }} \\
(\mathrm{cm} / \mathrm{s})\end{array}$ & $\begin{array}{c}\text { Static liquid } \\
\text { height }(\mathrm{cm})\end{array}$ & $\begin{array}{c}\text { Number of } \\
\text { gas injectors }\end{array}$ & $\begin{array}{c}\text { Aspect } \\
\text { ratio }\end{array}$ \\
\hline 11.2 & 1.0 & 110 & 2 & 9.8 \\
15.2 & 1.0 & 110 & 3 & 7.2 \\
32.0 & 1.2 & 110 & 6 & 3.4 \\
32.0 & 1.9 & 110 & 6 & 3.4 \\
\hline
\end{tabular}

\subsection{Notation}

$u_{c} \quad$ liquid velocity field

$u_{d} \quad$ gas velocity field

$\varepsilon_{c} \quad$ liquid volume fraction

$\varepsilon_{d} \quad$ gas volume fraction

$U_{\text {sup }} \quad$ superficial gas velocity

$d_{p} \quad$ bubble diameter

$\tau \quad$ surface tension

$\rho_{c} \quad$ liquid mass density

$\rho_{d} \quad$ gas mass density

$\mu_{c} \quad$ liquid viscosity

Eo Eotvos number $\equiv d_{p}^{2} \rho_{c} g / \tau$

Re bubble Reynolds number $\equiv d\left|u_{c}-u_{d}\right| \rho_{c} / \mu_{c}$ 


\subsection{Literature Cited}

Addessio, F. L., J. R. Baumgardner, J. K. Dukowicz, N. L. Johnson, B. A.Kashiwa, R. M. Rauenzahn, and C. Zemach, "CAVEAT: A Computer Code for Fluid Dynamics Problems with Large Distortion and Internal Slip", Los Alamos National Laboratory Report LA-10613MS, Rev. 1, (1992).

Baumgardner, J. R., M. C. Cline, N. L. Johnson, and B. A. Kashiwa, "CFDLIB: A Library of Computer Codes for Problems in Computational Fluid Dynamics", Los Alamos National Laboratory Report LA-UR-90-1361 (1990).

Biesheuvel, A. and S. Spoelstra, “The added mass coefficient of a dispersion of spherical gas bubbles in liquid," Int. J. Multiphase Flow 15, 911 (1989).

Batchelor, G. K., An Introduction to Fluid Dynamics. Cambridge University Press, Cambridge (1967).

Cranfill, C. W., "EOSPAC: A Subroutine Package for Accessing the Los Alamos Sesame EOS Data Library", Los Alamos National Laboratory Report LA-9728-M, UC-32, (1983).

Chen, J. J. J., M. Jamialahmadi and S. M. Li, “Effect of liquid depth on circulation in bubble columns: a visual study," Chem. Engng. Res. Des., 67, 203 (1989).

Chen, R. C. and L.-S. Fan, "Particle image velocimetry for characterizing the flow structure in three-dimensional gas-liquid-solid fluidized beds," Chem. Eng. Sci., 47, 3615 (1992).

Chen, R. C., J. Reese and L.-S. Fan, "'Flow structure in three-dimensional bubble column and three-phase fluidized beds," AIChE. J., 40, 1093 (1994).

Clift, R., Grace, J. R. and M. E. Weber, Bubbles, Drops, and Particles. Academic Press, New York, U.S.A. (1978).

Devanathan, N., M. P. DudukoviÊ, A. Lapin and A. Lubbert, "Chaotic flow in bubble column reactors," Chem. Eng. Sci., 50, 2661 (1995).

Drew, D. A. , "Mathematical modeling of two-phase flow", Ann. Rev. Fluid Mech., 15, 261 (1983).

Lin, T.-J, J. Reese, T. Hong and L.-S. Fan, “Quantitative analysis and computation of twodimensional bubble columns," AIChE. J., 42, 301 (1996).

Mudde, R. F., D.J. Lee, J. Reese and L.-S. Fan, "Role of coherent structures on Reynolds stresses in a 2-D bubble column," AIChE. J., 43, 913 (1997). 
Becker, S., A. Sokolichin and G. Eigenberger, 'Gas-liquid flow in bubble columns and loop reactors: part II. comparison of detailed experiments and flow simulations," Chem. Eng. Sci., 49, 5747 (1994).

Kashiwa, B. A., N. T. Padial, R. M. Rauenzahn, and W. B. VanderHeyden, "A Cell-Centered ICE Method for Multiphase Flow Simulations", Los Alamos National Laboratory Report LAUR-93-3922, (1993).

Kashiwa, B. A. and R. M. Rauenzahn, "A multimaaterial formalism," FED. Vol. 185, Numerical Methods in Multiphase Flows, ASME, 149-157 (1994).

Lapin, A. and A. Lubbert," Numerical simulation of the dynamics of two-phase gas-liquid flows in bubble columns," Chem. Eng. Sci., 49, 3361 (1994).

Lopez de Bertodano, M., R. T. Lahey, Jr. and O. C. Jones, "Development of $k-\varepsilon$ model for bubbly two-phase flow," ASME Journal of Fluids Engineering, 116, 128 (1994).

Lance, M., and J. Baraille, J., 'Turbulence in the liquid phase of a uniform bubbly air-water flow," J. Fluid Mech., 22, 95 (1991).

Katsumi, T., A. Furumoto, L.-S. Fang and J. Zhang, "Suspension viscosity and bubble rise velocity in liquid-solid fluidized beds," Chem. Eng. Sci., 52, 3053 (1997).

Delnoij, E., F. A. Lammers, J. A. M. Kuipers and W. P. M. van Swaaij, "Dynamic simulation of dispersed gas-liquid two-phase flows using a discrete bubble model," Chem. Eng. Sci., 52, 1429 (1997).

Delnoij, E., J. A. M. Kuipers and W. P. M. van Swaaij, "Computational fluid dynamics applied to gas-liquid contactors," Chem. Eng. Sci., 52, 3623 (1997a).

Delnoij, E., J. A. M. Kuipers and W. P. M. van Swaaij,"Dynamic simulation of gas-liquid twophase flow: effect of column aspect ration on the flow structure," Chem. Eng. Sci., 52, 3759 (1997b).

Sato, Y., and K. Sekoguchi, "Liquid velocity distribution in two-phase bubble flow," Int. J. Multiphase Flow, 2, 79 (1975).

Sato, Y., M. Sadatomi and K. Sekoguchi, "Momentum and heat transfer in two-phase bubble flow I," Int. J. Multiphase Flow, 7, 167 (1981).

Sokolichin, A and G. Eigenberger, "Gas-liquid flow in bubble columns and loop reactors: part I. Detailed modeling and numerical simulation," Chem. Eng. Sci., 49, 5735 (1994).

Svendsen, H. F., H. A. Jakobsen and R. Torvik, "'Local flow structures in internal loop and bubble column reactors," Chem. Eng. Sci., 47, 3297 (1992). 
Theofanous, T. G., and J. Sullivian, "Turbulence in two-phase disperse flow," J. Fluid Mech., 116, 343(1991).

Torvik, R. and H. F. Svendsen, "Modeling of slurry reactors: a fundamental approach," Chem. Eng. Sci., 45, 2325 (1990).

Prosperetti, A. and D. Z. Zhang, "Disperse phase stress in two-phase flow," Chem. Eng. Comm., 141-142, 387 (1996).

Prosperetti, A. and A. V. Jones, "Pressure forces in disperse two-phase flow", Int. J. Multiphase Flow, 10, 425 (1984).

Wallis, G. B., "The averaged Bernoulli equation and macroscopic equations of motion for the potential flow of two-phase dispersion," Int. J. Multiphase Flow, 17, 683 (1991).

Wijngaarden, L. van, "Hydrodynamics interactions between gas bubbles in liquid," J. Fluid Mech., 77, 27 (1976).

Yang, Y. B., N. Devannathan and M. P. Dudukovic, "Liquid backmixing in bubble columns via computer-automated radioactive particle tracking (CARPT)," Exp. Fluids, 16, 1 (1993).

Zhang, D. Z. and A. Prosperetti, "Ensemble phase-averaged equations for bubbly flows," Phys. Fluids 6, 2956 (1994).

Zhang, D. Z. and A. Prosperetti, "Momentum and energy equations for disperse two-phase flows and their closure for dilute suspensions," Int. J. Multiphase Flow, 23, 425 (1994).

Zhang, D. Z. and A. Prosperetti,"Averged Momentum equations for invisid disperse twophase flow," J. Fluid Mech, 267, 185 (1994).

Ranade, V. V., "Flow in bubble columns: some numerical experiments" Chem. Eng. Sci., 47, 1857 (1997).

Zuber, N., "On the dispersed two-phase flow in the larminar regime," Chem. Eng. Sci. 19, 897 (1964).

Webb, C., F. Que and P. R. Senior, 'Dynamics simulation of gas-liquid dispersion behavior in a 2-D bubble column using a graphics mini-super computer," Chem. Eng. Sci., 47, 3305 (1992). 


\section{SUMMARY AND CONCLUSIONS}

It is appropriate to summarize here the various tasks originally set for this study, the accomplishments achieved and the conclusions reached. Overall, all of the objectives set for this cooperative Industry-University grant have been achieved or exceeded.

The original goals set for this study, the related accomplishments and conclusions are listed below, grouped by individual tasks.

Task 1. Develop a computerized mathematical procedure (based on physics of radiation and Monte Carlo calculations) for calibration of the Computer Automated Radioactive Particle Tracking Technique (CARPT) that will eliminate the currently lengthy in-situ calibration and allow the technique to be used in the field on high pressure units. Specifically, plan the use of CARPT on a high pressure bubble column.

- Monte Carlo based procedure for calibration of detectors and for determination of radioactive particle position during CARPT runs has been developed and fully implemented. Details are described in Chapter 5 of this report.

- CARPT runs have been executed as planned at elevated pressure (Chapter 4 of this report). This necessitated the construction of a specialized facility at WU and of a novel particle positioning device for calibration purposes.

- As a result of the availability of the Monte Carlo procedure, future CARPT runs at elevated pressures will be much easier to implement and will be more accurate.

- The basic liquid recirculation flow pattern is apparently unaffected by pressure. In the time averaged sense a single liquid recirculation cell is observed, with the liquid rising in the middle of the column and falling by the walls. The magnitude of liquid velocities is diminished at elevated pressure.

Task 2. Improve the accuracy of the CARPT technique and compare the velocities obtained by CARPT in certain regions of the column to those determined by the Heat Transfer Probe and Particle Image Velocimetry (PIV).

- The accuracy of the CARPT technique, especially for evaluation of instantaneous velocities, was greatly improved by implementation of a wavelet based filter (Chapter III of the First Annual Technical Report) and by the Monte Carlo technique (Chapter 5 in this report).

- A thorough comparison of PIV and CARPT data in a 4" diameter column at superficial gas velocity and low holdup, where PIV results can be deemed very accurate, validated fully CARPT measurements (Chapter 4 of the Second Annual Technical Report). 
- Comparison of CARPT data with Laser Doppler Anemometry (LDA) and Hot Film Anemometry (HFA) indicate good agreement (Chapter 6 of Second Annual Technical Report).

-• Extensive comparison of various techniques revealed that at low superficial gas velocities all of them can provide good results. At such conditions of low gas holdup PIV, LDA and HFA have the capabilities of revealing fine structures of the flow and dynamic behavior at high frequencies, which are the features which CARPT lacks. However, CARPT always provides an accurate description of the mean flow quantities and of the dynamic features of the large flow structures. This information is obtainable by CARPT at very high superficial gas velocities when, due to very high gas holdup, all other techniques fail. The heat transfer probe (HP) which also can be used at high superficial gas velocities underestimates the mean velocities such conditions.

Task 3. Develop local probes for accessing velocities, holdups and heat transfer coefficients in bubble column reactors.

- A special heat transfer probe was developed (OSU) and tested (ER\&E) to measure the instantaneous heat transfer coefficient due to gas bubbles injected in liquid and liquid-solid systems (Chapter IV of the First Annual Technical Report).

- A light transmittance probe was developed (OSU) and tested to quantify the bubble characteristics and frequencies of bubble passage (Chapter 7 of the Second Technical Report).

- A high framing, high resolution, high capacity PIV system was developed for the purposes of this study at OSU (Chapter 5 of the Second Annual Technical Report).

- A computer tomography system (CT) was implemented to obtain gas holdup profiles at different gas superficial velocities (Chapter 3 of Second Annual Technical Report, Chapter 3 of this report).

- Reliable time averaged density profiles can be obtained by gamma ray based in bubble columns.

- Local heat transfer coefficients can be assessed effectively with the developed probe.

- $\quad$ PIV and optical probes are valuable research tools for quantification of bubbly flows.

Task 4. Collect velocity and voidage profile data in gas-liquid and gas-liquid-solid systems at different solids loadings and at close to atmospheric pressure. 
- The collected velocity and holdup profiles were used for validation of the various techniques such as in CARPT-PIV comparisons (Chapter 5 of Second Annual Technical Report), for comparison of CFD predictions and models (Chapter 6 of Second Technical Report and Chapter 7 of this report) and for augmentation of the data base for evaluation of important fluid dynamic parameters (Chapter 6 of this report).

- A very useful correlation has been developed at OSU for prediction of bubble rise velocity. The validity of this correlation in high pressure, high temperature systems was validated (Chapter 6 of this report).

Task 5. Use state-of-the-art hydrodynamic models and codes to predict velocity and holdup fields under conditions studied experimentally. Search for most suitable constitutive forms (e.g., lift, drag, turbulence closure models, etc.) to reach agreement between calculated and experimentally observed values. Try to assess the effect of elevated operating pressure on various constitutive forms.

- A fundamentally based computational method was developed at OSU based on the volume averaged method, the dispersed particle method, and the volume of fluid to simulate the transient behavior of a rising bubble in gas-liquid-solid (Chapter 6 of this report).

- The Euler-Euler two-fluid model, in the framework of the Los Alamos CFDLIB code, was adopted for simulation of flow fields in the whole bubble column. Mixing length and bubble induced turbulence closures were tried (Chapter 9 of Second Annual Technical Report and Chapter 7 of this report).

- As an interim measure an engineering phenomenological model was developed for both liquid and gas flow and mixing based on the collected data base on this and related studies. This model captures the essence of the observed recirculation and mixing phenomena (Chapter 8 of the Second Annual Technical Report).

-- The fundamental model at OSU predicts well the bubble shape, trajectory and rise velocity in slurries.

- The Euler-Euler model predicts well the time averaged quantities and dynamic features of large structures in bubbly flows (2D columns).

Task 6. Collect the data in a high pressure 6" diameter bubble column and compare to model predictions. Also collect high pressure PIV data in slurry systems at Ohio State University. Refine the models if needed.

- Instead of collecting high pressure data at the Florham Park Exxon Research and Engineering facility, an equivalent experimental set-up was developed, with additional funding from ER\&E, at Washington University. This change in plans was necessitated by the safety and radioactive material licensing issues which prevented the work to be conducted at Exxon facilities. The fact that a high 
pressure 6" bubble column facility had to be designed and constructed at WU necessitated the 9 month (no-cost to DOE) extension of this research. High pressure data for gas holdup and velocity were conducted in the 6" column at WU (Chapter 3 and 4 of this report).

- High pressure data in smaller diameter columns was also collected via PIV and other techniques at OSU and used for development of correlations for various fluid dynamic parameters (Chapter 6 of this report).

- An improved understanding emerged about the behavior of high pressure bubble columns and slurry bubble columns. High pressure delays the transition to churn turbulent flow, flattens the observed gas holdup profiles at high gas superficial velocity and reduces the magnitude of liquid recirculation.

All of the above accomplishments bring us closer to our goal of improved quantitative understanding of fluid dynamics in bubble columns. The progress in this University-Industry joint project was greatly helped and assisted by the complementary companion DOE subcontract via Air Products to both Washington University and Ohio State University. The contract DE FC 95 22 PC 95051 is gratefully acknowledged. 